TRANSACTIONS OF THE

AMERICAN MATHEMATICAL SOCIETY

Volume 352, Number 1, Pages 311-362

S 0002-9947(99)02118-2

Article electronically published on September 9, 1999

\title{
FUCHSIAN GROUPS, QUASICONFORMAL GROUPS, AND CONICAL LIMIT SETS
}

\author{
PETER W. JONES AND LESLEY A. WARD
}

\begin{abstract}
We construct examples showing that the normalized Lebesgue measure of the conical limit set of a uniformly quasiconformal group acting discontinuously on the disc may take any value between zero and one. This is in contrast to the cases of Fuchsian groups acting on the disc, conformal groups acting discontinuously on the ball in dimension three or higher, uniformly quasiconformal groups acting discontinuously on the ball in dimension three or higher, and discrete groups of biholomorphic mappings acting on the ball in several complex dimensions. In these cases the normalized Lebesgue measure is either zero or one.
\end{abstract}

\section{INTRODUCTION}

A classical result of Hopf says that the normalized one-dimensional Lebesgue measure of the conical limit set of any finitely generated Fuchsian group is either zero or one. This has been extended to infinitely generated Fuchsian groups, to conformal and to uniformly quasiconformal groups acting discontinuously on the unit ball in (real) dimension three or higher, and to discrete groups of complex hyperbolic isometries of the unit ball in several complex variables. In this article it is shown that this dichotomy does not hold for uniformly quasiconformal groups acting on the unit disc in the complex plane. For each $\lambda$ with $0 \leq \lambda \leq 1$ we explicitly construct a quasiconformal group, acting on the disc, whose conical limit set has normalized one-dimensional Lebesgue measure equal to $\lambda$. The main step is to construct a doubling measure supported on the conical limit set of a certain Fuchsian group, where this conical limit set has Lebesgue measure zero.

A group $G$ of homeomorphisms of the unit disc, in dimension two, or of the unit ball, in dimension greater than two, is said to act discontinuously if each point in the disc or ball has a neighborhood $U$ such that only finitely many of the images $g(U)$, $g \in G$, intersect $U$. The limit set $L(G)$ of such a group is the set of accumulation points of the orbit of the origin under the action of $G$. The same limit set is obtained using any point in the disc in place of the origin. The discontinuity of $G$ implies that $L(G)$ is a subset of the unit circle or sphere. A point $x \in L(G)$ is a conical limit point of $G$ if there is a sequence of orbit points which converges to $x$ inside a Euclidean cone with vertex at $x$, axis perpendicular to the circle or sphere, and opening angle less than $\pi / 2$, so that the sides of the cone are not tangent to the

Received by the editors February 1, 1996 and, in revised form, May 13, 1997.

1991 Mathematics Subject Classification. Primary 30C62, 30F35, $20 \mathrm{H} 10$.

Key words and phrases. Fuchsian groups, quasiconformal groups, limit sets.

Partially supported by NSF grant \#DMS-92-13595.

Research at MSRI supported in part by NSF grant \#DMS-90-22140. 
circle or sphere. The conical limit set $L_{c}(G)$ of $G$ is the set of conical limit points of $G$.

A Fuchsian group is a group of Möbius transformations which acts discontinuously on the unit disc $\mathbb{D}$ in the complex plane. For simplicity we assume that the group has no elliptic elements. Hopf $[\mathrm{H}]$ showed that the conical limit set of a finitely generated Fuchsian group has normalized Lebesgue measure zero or one. This depends on whether the Poincaré series for the group converges or diverges at the exponent 1:

$$
\left|L_{c}(G)\right|= \begin{cases}0, & \text { if } \sum_{g \in G}(1-|g(0)|)<\infty ; \\ 1, & \text { if } \sum_{g \in G}(1-|g(0)|)=\infty ;\end{cases}
$$

the result also holds for infinitely generated Fuchsian groups.

For Fuchsian groups, and for groups of Möbius transformations acting discontinuously on the $n$-dimensional ball when $n \geq 3$, there are several dichotomies which are equivalent to the zero-one dichotomy for the normalized $(n-1)$-dimensional measure of the conical limit set. The equivalence of the following conditions was proved by Sullivan [S1, S2]:

1. $\left|L_{c}(G)\right|=0$;

2. the Poincaré series converges at the exponent $n-1: \sum_{g \in G}(1-|g(0)|)^{n-1}<\infty$;

3. Green's function for the Laplace-Beltrami operator exists for the hyperbolic manifold $\mathbb{B}^{n} / G$; and

4. the geodesic flow on $\mathbb{B}^{n} / G$ is transient.

Otherwise, the conical limit set has full Lebesgue measure in the $(n-1)$-sphere; the Poincaré series diverges at the exponent $n-1$; there is no Green's function for $\mathbb{B}^{n} / G$ (that is, the integral of the heat kernel for $\mathbb{B}^{n} / G$ does not converge); and the geodesic flow on $\mathbb{B}^{n} / G$ is recurrent and completely ergodic. See Nicholls' book [N2] for further information. Analogous results hold in several complex variables [Kam1, Kam2, MW].

A homeomorphism $\varphi: \Omega \rightarrow \Omega^{\prime}$ of complex domains is called $K$-quasiconformal if it is in the Sobolev class $W_{\text {loc }}^{1,2}$ and its directional derivatives satisfy

$$
\max _{\alpha}\left|\partial_{\alpha} \varphi(z)\right| \leq K \min _{\alpha}\left|\partial_{\alpha} \varphi(z)\right|
$$

for almost every $z \in \Omega$. Geometrically, this means that for almost every $z \in \Omega$, infinitesimal circles centred at $z$ are mapped by $\varphi$ to infinitesimal ellipses centred at $\varphi(z)$, whose eccentricities are uniformly bounded below by $1 / K$. A quasiconformal group is a group of $K$-quasiconformal maps acting discontinuously on the disc, for some fixed $K \geq 1$.

One can define quasiconformal mappings in dimension $n \geq 3$ by generalizing the geometric definition above, using spheres and ellipsoids instead of circles and ellipses. Garnett, Gehring, and Jones [GGJ], and independently Tukia [T3], showed that results analogous to Hopf's on the measure of the conical limit set and the convergence of the Poincaré series at the exponent $n-1$ hold for uniformly quasiconformal groups when the dimension is at least three: If $G$ is a group of $K$ quasiconformal mappings acting discontinuously on the $n$-dimensional unit ball, where $n \geq 3$, then

$$
\left|L_{c}(G)\right|= \begin{cases}0, & \text { if } \sum_{g \in G}(1-|g(0)|)^{n-1}<\infty ; \\ 1, & \text { if } \sum_{g \in G}(1-|g(0)|)^{n-1}=\infty .\end{cases}
$$


In this article we show that, in sharp contrast to the higher dimensional case, there is no zero-one dichotomy for the normalized Lebesgue measure of the conical limit set of a quasiconformal group acting on the two-dimensional unit disc.

Theorem 1.1. For each number $\lambda \in[0,1]$, there is a quasiconformal group $\Gamma$ acting on the unit disc such that the normalized one-dimensional Lebesgue measure of the conical limit set of $\Gamma$ is $\lambda$.

A doubling measure $\mu$ on the circle is a positive measure satisfying $\mu(\widetilde{I}) \leq c \mu(I)$, for some constant $c>0$, whenever $I$ is an arc of the circle and $\widetilde{I}$ is the arc with the same centre and twice the length. Equivalently, $\mu(I) \leq c^{\prime} \mu(J)$ for some uniform constant $c^{\prime}>0$ whenever $I$ and $J$ are adjacent arcs of equal length.

Theorem 1.1 is a consequence of the following result:

Theorem 1.2. There exist a Fuchsian group $G$ and a doubling measure $\mu$ such that the conical limit set of $G$ has one-dimensional Lebesgue measure zero, and $\mu$ is supported on the conical limit set of $G$.

Our result is perhaps rather surprising given that Sullivan [S2] and independently Tukia [T1] have proven that every $K$-quasiconformal group acting on the disc is conjugate by a quasiconformal mapping to a Möbius group. The corresponding statement is false in higher dimensions [T2]. On the other hand, boundary values of quasiconformal homeomorphisms of the disc (in other words, quasisymmetric maps) have much less regularity than is true in higher dimensions. If $\varphi: \mathbb{B}^{n} \rightarrow \mathbb{B}^{n}$ is $K$-quasiconformal and $n \geq 3$, the restriction of $\varphi$ to $\partial \mathbb{B}^{n}$ is also $K$-quasiconformal and hence lies in the Sobolev space $W^{1, n-1}\left(\partial \mathbb{B}^{n}\right)$. In particular $\left.\varphi\right|_{\partial \mathbb{B}^{n}}$ takes sets of $(n-1)$-dimensional Lebesgue measure zero to sets of $(n-1)$-dimensional Lebesgue measure zero. Quasisymmetric mappings do not have this property. It is this lack of regularity for quasisymmetric mappings that we exploit to construct our examples.

Theorem 1.1 is an easy consequence of Theorem 1.2. This argument, carried out in Section 3, uses little more than the Beurling-Ahlfors theorem: A homeomorphism $f$ of the circle is the restriction of a quasiconformal mapping of the disc to itself if and only if the distributional derivative $\frac{\partial f}{\partial \theta}$ on the circle is a doubling measure.

Theorem 1.2 gives some information about the possible geometry of limit sets and of sets which support doubling measures. Roughly speaking, the support of a doubling measure must be rather evenly distributed. We give a Fuchsian group whose conical limit set is evenly enough distributed to support a doubling measure, even though it has zero Lebesgue measure. (In fact, the Hausdorff dimension of this conical limit set is strictly less than one.) We construct a doubling measure that is tailored so its support is in this small set. Such constructions were first carried out by Kahane $[\mathrm{K}]$, and our method follows his philosophy, though the details are necessarily more complicated.

As José Fernández has pointed out to us, the Patterson-Sullivan measure associated to the group $G$ is a natural candidate for the doubling measure of Theorem 1.2. Unfortunately, certain technical difficulties are encountered in this approach, and we have not yet understood how to overcome them.

It is interesting to note that the result of Theorem 1.2 cannot be achieved if the quasisymmetric map associated to the doubling measure $\mu$ is in the Teichmüller space of the group $G$, or if $G$ is finitely generated. The first statement follows from the fact that the existence of Green's function is a quasiconformal invariant. If $G$ is finitely generated and of the first kind, then $L_{c}(G)$ has full measure in the unit 
circle. If $G$ is finitely generated and of the second kind, then $\partial \mathbb{D} \backslash L_{c}(G)$ contains an arc, and so $L_{c}(G)$ cannot support a doubling measure.

Section 4 contains a brief summary of our proof of Theorem 1.2. A detailed outline of the proof, including a full model calculation, can be found in MSRI Preprint No. 1996-024.

The rest of this paper is organized as follows. In Section 2 we give some definitions and notation. In Section 3, we prove Theorem 1.1 as a corollary of Theorem 1.2. In Section 5 we establish some basic properties of the Fuchsian group $G$ used in our proof of Theorem 1.2. In Section 6, we prove that a certain simple construction yields doubling measures which are supported on small sets. Sections 7 to 11 contain the construction of our doubling measure, up to the specification of some parameters. In Section 7 we fix a set of fundamental domains in the universal cover $\mathbb{D}$ of the Riemann surface $\mathbb{D} / G$. In Section 8 we define a collection of Whitney intervals in the Riemann surface, and establish some properties of its lift to the universal cover. In Section 9 we construct a grid of intervals in the circle, on which our doubling measure will be built. Section 10 contains estimates which control the geometric distortion caused by the covering map. In Section 11 we define a sequence of density functions which, by the lemma in Section 6 , yields a doubling measure. In Section 12 we define some auxiliary functions and outline the rest of the proof. Sections 13 and 14 contain estimates of the expectation and second moment, with respect to the doubling measure, of the auxiliary functions. In Section 15, an argument involving a constrained random walk on the Riemann surface, together with the estimates of the previous two sections, proves that the measure is supported on the conical limit set of $G$.

It is a pleasure for the second author to acknowledge the support of MSRI during the time when this manuscript was being prepared for publication.

\section{Definitions AND NOtATion}

The conical limit set is also known as the radial or non-tangential limit set. Hedlund $[\mathrm{He}]$ introduced conical limit points in connection with his study of horocyclic transitive points. The conical limit set may be characterized in terms of spherical caps. A spherical cap on a point $x$ in the unit disc $\mathbb{D}$ is an arc of the unit circle $\partial \mathbb{D}$ of the form

$$
\operatorname{Cap}(x, a)=\{y \in \partial \mathbb{D}|| y-x \mid \leq a(1-|x|)\},
$$

where $1<a<\infty$. A non-tangential cone on a point $y \in \partial \mathbb{D}$ is a subset of $\mathbb{D}$ of the form

$$
\text { Cone }(y, b)=\{x \in \mathbb{D}|| y-x \mid \leq b(1-|x|)\},
$$

where $1<b<\infty$. Clearly $y \in \operatorname{Cap}(x, a)$ if and only if $x \in \operatorname{Cone}(y, a)$. Nontangential cones are comparable to the Euclidean cones discussed in the introduction, in the sense that each such Euclidean cone contains a non-tangential cone and is contained in a non-tangential cone. It follows that $y$ is the non-tangential limit of $\left\{g_{j}(0)\right\}_{j=1}^{\infty}$ if and only if there is an $a$ such that $y$ lies in infinitely many spherical caps Cap $\left(g_{j}(0), a\right)$. Taking a countable union over wider and wider opening angles of the cones, the conical limit set is given by

$$
L_{c}(G)=\bigcup_{l=2}^{\infty} \bigcap_{k=1}^{\infty} \bigcup_{j=k}^{\infty} \operatorname{Cap}\left(g_{j}(0), l\right) .
$$


A set $K$ in the complex plane is uniformly perfect if there is a constant $c>0$ such that, for each $z_{0} \in K$ and for all $r$ such that $0<r<\operatorname{diam} K$,

$$
K \cap\left\{z|c r \leq| z-z_{0} \mid \leq r\right\} \neq \emptyset .
$$

(Here diam $K$ is the diameter of $K$.) In other words, there is an upper bound on the moduli of annuli lying in the complement of $K$.

The Poincaré series for a discrete group $G$ is the series

$$
\sum_{g \in G}(1-|g(0)|)^{s}
$$

where $s$ is positive. There is a critical exponent $\delta=\delta(G)$, called the exponent of convergence, such that the series converges for all $s>\delta$ and diverges for all $s<\delta$.

Let $\Omega$ be a domain in $\overline{\mathbf{C}}$, let $E$ be a Borel subset of $\partial \Omega$, and let $z$ be a point in $\Omega$. The harmonic measure at $z$ of $E$ in $\Omega$ is the Perron solution $w(z)=w(z, E, \Omega)$ of the Dirichlet problem in $\Omega$ for the boundary values $\mathbf{1}_{E}$. As a function of $z$, $w(z, E, \Omega)$ is harmonic in $\Omega$. For fixed $z, w(z, E, \Omega)$ is a probability measure on $\partial \Omega ; w(z, E, \Omega)$ is the probability that a Brownian traveller from $z$ first hits $\partial \Omega$ in the set $E$. Harmonic measure is monotonic in the domain $\Omega$ : if $z \in \Omega_{1} \subset \Omega_{2}$ and $E \subset \partial \Omega_{1} \cap \partial \Omega_{2}$, then $w\left(z, E, \Omega_{1}\right) \leq w\left(z, E, \Omega_{2}\right)$. It is also monotonic in $E$ : if $E_{1} \subset E_{2} \subset \partial \Omega$, then $w\left(z, E_{1}, \Omega\right) \leq w\left(z, E_{2}, \Omega\right)$.

The hyperbolic metric on the disc $\mathbb{D}$ is given by the element of arclength $d s=$ $\frac{2}{1-|z|^{2}}|d z|$. The Möbius transformations $g: \mathbb{D} \rightarrow \mathbb{D}$ are the isometries for this metric. They are of the form

$$
g(z)=e^{i \theta} \frac{z-z_{0}}{1-\bar{z}_{0} z}
$$

where $\theta \in[0,2 \pi]$ and $z_{0} \in \mathbb{D}$. The hyperbolic geodesics are the orthocircular arcs: that is, arcs of circles which meet the unit circle $\partial \mathbb{D}$ at right angles. The hyperbolic distance from 0 to $a \in \mathbb{D}$ is

$$
d_{\text {hyp }}(0, a)=\int_{0}^{|a|} \frac{2}{1-|z|^{2}}|d z|=\log \left[\frac{1+|a|}{1-|a|}\right] .
$$

Let $\Omega$ be a domain in $\overline{\mathbf{C}}$ whose universal covering space is the disc, and let $\pi: \mathbb{D} \rightarrow \Omega$ be the covering map. The hyperbolic metric on $\mathbb{D}$ can be projected via $\pi$ to a metric on $\Omega$ given by $\lambda_{\Omega}(w)|d w|=\lambda_{\Omega}(\pi(z))\left|\pi^{\prime}(z)\right||d z|=\frac{2}{1-|z|^{2}}|d z|$, where $w=\pi(z)$. The boundary $\partial \Omega$ of $\Omega$ is uniformly perfect if and only if there is a constant $c_{\Omega}>0$ such that the function $\lambda_{\Omega}$ satisfies

$$
\frac{c_{\Omega}}{\operatorname{dist}(w, \partial \Omega)} \leq \lambda_{\Omega}(w) \leq \frac{2}{\operatorname{dist}(w, \partial \Omega)} .
$$

See [BP, Po2]. Here dist denotes Euclidean distance.

In dimension two, there is a connection between quasiconformal maps and doubling measures. Let $\varphi: \mathbf{C} \rightarrow \mathbf{C}$ be a $K$-quasiconformal map which preserves the upper half plane. Then $\varphi$ maps $\mathbb{R}$ to itself, and the restriction $f$ of $\varphi$ to $\mathbb{R}$ is an increasing homeomorphism which satisfies

$$
\frac{1}{M} \leq \frac{f(x+t)-f(x)}{f(x)-f(x-t)} \leq M
$$

with a constant $M$ depending on $K$, for all $x \in \mathbb{R}$ and all $t>0$. Functions with this property are called $M$-quasisymmetric. Quasisymmetry is a necessary and sufficient 
condition for an increasing homeomorphism $f$ of $\mathbb{R}$ to be the boundary values of a quasiconformal mapping which preserves the upper half plane [BA]. Note that different quasiconformal mappings may have the same boundary values.

Let $f: \mathbb{R} \rightarrow \mathbb{R}$ be an increasing homeomorphism. Define a measure $\mu$ on $\mathbb{R}$ by setting $\mu([a, b])=f(b)-f(a)$ for intervals $[a, b]$. Then $f$ is $M$-quasisymmetric if and only if $\mu$ satisfies $1 / M \leq \mu(I) / \mu(J) \leq M$ whenever $I$ and $J$ are adjacent intervals of equal length. In other words, $f$ is $M$-quasisymmetric if and only if $\mu$ is doubling.

To summarize, there is a many-to-one correspondence between quasiconformal self-mappings of the upper half plane and doubling measures on the real line. There is a similar correspondence between quasiconformal self-mappings of the disc and doubling measures on the circle.

Some references for the material above are $[\mathrm{N} 1]$ and $[\mathrm{L}]$.

Two quantities $A$ and $B$ are comparable, denoted $A \sim B$, if there is a constant $c>0$ such that $\frac{1}{c} B \leq A \leq c B$. We also write $A \stackrel{c}{\sim} B$ if $A$ and $B$ are comparable with constant $c$. Throughout the paper we normalize Lebesgue measure so that the unit $n$-sphere has measure one.

In the figures we denote the unit circle $\partial \mathbb{D}$ by $S^{1}$.

\section{Proof of Theorem 1.1 assuming Theorem 1.2}

Fix a number $\lambda$ with $0 \leq \lambda \leq 1$. Let $\mu$ be the measure given by Theorem 1.2, and take $c>0$ such that $\mu(2 I) \leq c \mu(I)$ for all $\operatorname{arcs} I$ in the unit circle $\partial \mathbb{D}$. Set $\nu(\cdot)=\lambda \mu(\cdot)+(1-\lambda)|\cdot|$, where $|\cdot|$ denotes normalized Lebesgue measure on $\partial \mathbb{D}$. Then $\nu$ is a doubling measure on the circle since, for each arc $I$ in $\partial \mathbb{D}$,

$$
\begin{aligned}
\nu(2 I) & =\lambda \mu(2 I)+(1-\lambda)|2 I| \\
& \leq c \lambda \mu(I)+2(1-\lambda)|I| \\
& \leq \max (c, 2) \nu(I) .
\end{aligned}
$$

The measure $\nu$ assigns the fraction $\lambda$ of its mass to the conical limit set of $G$.

Define $f: \partial \mathbb{D} \rightarrow \partial \mathbb{D}$ so that $\nu([a, b])=f(b)-f(a)$ for all arcs $[a, b]$ in $\partial \mathbb{D}$. $f$ is a quasisymmetric mapping of the circle to itself, and $|f(E)|=\nu(E)$ for any measurable subset $E$ of the circle. The map $f$ may be extended to a quasiconformal mapping $\varphi$ of the unit disc onto itself [BA, DE] and further to a quasiconformal self-mapping of $\overline{\mathbf{C}}$. Conjugating the Fuchsian group $G$ of Theorem 1.2 by $\varphi$ yields a quasiconformal group $\Gamma=\varphi \circ G \circ \varphi^{-1}$ which acts on the disc.

It remains to show that the conical limit set of $\Gamma$ is the image under $\varphi$ of the conical limit set of the Fuchsian group $G$. This is a special case of a much more general fact; see $[\mathrm{M}]$ for example. We give a direct proof. It is sufficient to show that a quasiconformal map $\varphi: \mathbb{D} \rightarrow \mathbb{D}$ maps any non-tangential cone into another non-tangential cone, perhaps with larger opening angle. Since $\varphi^{-1}$ is also quasiconformal, this implies that $L_{c}(\Gamma)=\varphi\left(L_{c}(G)\right)$.

Define the cross ratio of any four distinct points $\alpha, \beta, \gamma$, and $\delta$ in $\overline{\mathbf{C}}$ by

$$
\tau=|\alpha, \beta, \gamma, \delta|=\frac{|\alpha-\gamma|}{|\alpha-\delta|} \cdot \frac{|\beta-\delta|}{|\beta-\gamma|},
$$

with the convention that if $\delta=\infty$,

$$
|\alpha, \beta, \gamma, \infty|=\frac{|\alpha-\gamma|}{|\beta-\gamma|}
$$


Let $\varphi: \overline{\mathbf{C}} \rightarrow \overline{\mathbf{C}}$ be $K$-quasiconformal. Let $\varphi(\tau)=|\varphi(\alpha), \varphi(\beta), \varphi(\gamma), \varphi(\delta)|$. Väisälä has shown $[\mathrm{V}]$ that there is an increasing function $\Phi_{K}:(0, \infty) \rightarrow(0, \infty)$, depending only on $K$, such that $\varphi(\tau) \leq \Phi_{K}(\tau)$ for all $\tau$.

Let $z$ be a point in Cone $(y, a)=\{z \in \mathbb{D}|| z-y \mid \leq a(1-|z|)\}$, where $y$ is a point in $\partial \mathbb{D}$ and $1<a<\infty$. Let $w$ be the point in $\partial \mathbb{D}$ such that $1-|\varphi(z)|=|\varphi(w)-\varphi(z)|$.

Suppose $\varphi^{-1}(\infty)=\infty$. Then

$$
\begin{aligned}
\frac{|\varphi(y)-\varphi(z)|}{1-|\varphi(z)|} & =\frac{|\varphi(y)-\varphi(z)|}{|\varphi(w)-\varphi(z)|} \\
& =|\varphi(y), \varphi(w), \varphi(z), \infty| \\
& \leq \Phi_{K}(|y, w, z, \infty|) \\
& =\Phi_{K}\left(\frac{|y-z|}{|w-z|}\right) \\
& \leq \Phi_{K}\left(\frac{|y-z|}{1-|z|}\right) \\
& \leq \Phi_{K}(a) .
\end{aligned}
$$

Here the second last inequality holds because $|w-z| \geq 1-|z|$ and $\Phi_{K}$ is increasing. Therefore $\varphi(z)$ lies in Cone $\left(\varphi(y), \Phi_{K}(a)\right)$.

Now suppose $\varphi^{-1}(\infty) \neq \infty$. Let $w$ and $y$ be any two points in the unit circle. Since $\varphi^{-1}(\infty)$ is not in the closed unit disc, we have

$$
\left|w-\varphi^{-1}(\infty)\right| \leq \frac{2+d}{d}\left|y-\varphi^{-1}(\infty)\right|
$$

where $d=\operatorname{dist}\left(\varphi^{-1}(\infty), \overline{\mathbb{D}}\right)>0$. Then

$$
\begin{aligned}
\frac{|\varphi(y)-\varphi(z)|}{1-|\varphi(z)|} & =|\varphi(y), \varphi(w), \varphi(z), \infty| \\
& \leq \Phi_{K}\left(\left|y, w, z, \varphi^{-1}(\infty)\right|\right) \\
& =\Phi_{K}\left(\frac{|y-z|}{|w-z|} \cdot \frac{\left|w-\varphi^{-1}(\infty)\right|}{\left|y-\varphi^{-1}(\infty)\right|}\right) \\
& \leq \Phi_{K}\left(\frac{|y-z|}{1-|z|} \cdot \frac{2+d}{d}\right) \\
& \leq \Phi_{K}\left(a \cdot \frac{2+d}{d}\right) .
\end{aligned}
$$

Hence $\varphi(z)$ lies in Cone $\left(\varphi(y), \Phi_{K}\left(a \cdot \frac{2+d}{d}\right)\right)$.

It follows that $L_{c}(\Gamma)=\varphi\left(L_{c}(G)\right)$, and $\left|L_{c}(\Gamma)\right|=\left|\varphi\left(L_{c}(G)\right)\right|=\nu\left(L_{c}(G)\right)=\lambda$. In other words $\Gamma$ is a quasiconformal group, acting on the unit disc, whose conical limit set supports the fraction $\lambda$ of the mass of Lebesgue measure on the unit circle. This completes the proof of Theorem 1.1.

\section{A brief outline of the Proof of Theorem 1.2}

Theorem 1.2. There exist a Fuchsian group $G$ and a doubling measure such that the conical limit set $L_{c}(G)$ of $G$ has one-dimensional Lebesgue measure zero, and the doubling measure is supported on $L_{c}(G)$. 
In this section we summarize the proof of Theorem 1.2. A much fuller outline, including a full model calculation, can be found in the MSRI preprint (No. 1996024) of this paper.

Let $K \subset[0,1]$ be the ternary Cantor set, and let $\Omega=\overline{\mathbf{C}} \backslash K$ be the complement of $K$ in the extended complex plane. Let $G$ be the Fuchsian group, acting on the disc $\mathbb{D}$, which uniformizes $\Omega$. In other words, $G$ is the covering group of $\Omega$ and $\mathbb{D} / G$ is conformally equivalent to $\Omega$. Let $\pi: \mathbb{D} \rightarrow \Omega$ be the covering map, normalized so that $\pi(0)=\infty$. The conical limit set $L_{c}(G)$ is the set of nontangential accumulation points of the orbit $\{g(0)\}_{g \in G}$. For this group $G$, the conical limit set has measure zero. (See Section 5.) Our aim is to construct a doubling measure which is supported on $L_{c}(G)$.

We make a Whitney decomposition of the complement of the Cantor set $K$ in the real line. We construct a tree in the disc, whose vertices are the lifts of the Whitney intervals via the covering map. The adjacency relations in the tree, in other words which pairs of vertices are connected by edges, are determined by a construction on the Riemann surface $\Omega$. We obtain the measure $\mu$ as the hitting probability, on the circle, of a random walk on the tree.

The main issue is to show that it is possible to fix the probabilities of the individual steps along the edges of the tree so that (1) the hitting probability measure is doubling, and (2) it is supported on the conical limit set of $G$.

\section{Choice of the Fuchsian group}

Let $K \subset[0,1]$ be the ternary Cantor set. Let $\Omega=\overline{\mathbf{C}} \backslash K$ be the complement of $K$ in the extended complex plane. $\Omega$ is an infinitely connected planar Riemann surface. Since $K=\partial \Omega$ contains more than two points, the universal covering space of $\Omega$ is the unit disc $\mathbb{D}$, and $\Omega$ is conformally equivalent to $\mathbb{D} / G$, where $G$ is a Fuchsian group. We choose this infinitely generated Fuchsian group $G$ as our example.

In this section we show that the conical limit set $L_{c}(G)$ of $G$ has one-dimensional Lebesgue measure zero, and moreover the Hausdorff dimension of $L_{c}(G)$ satisfies $1 / 2 \leq \operatorname{dim}\left(L_{c}(G)\right)<1$. The remainder of the paper is devoted to the construction of a doubling measure which is supported on $L_{c}(G)$.

Since $K$ has positive logarithmic capacity, Green's function exists for $\Omega$, and the result cited in the introduction on the measure of the conical limit set of a Fuchsian group implies that $\left|L_{c}(G)\right|=0$. Fernández $[\mathrm{F}]$ has shown that if the boundary $\partial \Omega$ of a planar domain $\Omega$ is uniformly perfect, then the exponent of convergence of the corresponding Fuchsian group is strictly less than one. The Cantor set $K$ is uniformly perfect. For Fuchsian groups the exponent of convergence is equal to the Hausdorff dimension of the conical limit set [Pa1, S1]. (See also [BJ], where this is proved in much greater generality.) We may therefore conclude that in fact $\operatorname{dim}\left(L_{c}(G)\right)<1$.

A Fuchsian group $G$ is of fully accessible type if there is a measurable fundamental set $B \subset \partial \mathbb{D}$ for the action of $G$ on $\partial \mathbb{D}$. In other words, $B$ contains no two $G$-equivalent points, and $\partial \mathbb{D}$ is a.e. equal to the union of the $G$-images of $B$ : $\sum_{g \in G}|g(B)|=1$. $G$ is of accessible type if there is a measurable set $B \subset \partial \mathbb{D}$, containing no two $G$-equivalent points, such that $\sum_{g \in G}|g(B)|>0$.

The normal fundamental domain $\mathcal{F}_{0}$ (see Section 7 ) for $\Omega=\overline{\mathbf{C}} \backslash K$ has the property that $\left|\partial \mathcal{F}_{0} \cap \partial \mathbb{D}\right|=0$. We outline the well-known proof. The covering 
map $\pi$ takes $\partial \mathcal{F}_{0}$ to $[0,1]$. Since $\partial \mathcal{F}_{0}$ is a rectifiable curve, $\pi$ preserves sets of zero length. (This is the F. and M. Riesz theorem; see [Po3].) Since $K$ has Lebesgue measure zero, $\partial \mathcal{F}_{0} \cap \pi^{-1}(K)=\partial \mathcal{F}_{0} \cap \partial \mathbb{D}$ has measure zero.

This implies that the group $G$ is not of accessible type [Po1]. On the other hand, Patterson [Pa2] showed that if $\delta(G)<1 / 2$, then $G$ is of fully accessible type. It follows that our group $G$ must have $\delta(G)=\operatorname{dim}\left(L_{c}(G)\right) \geq 1 / 2$.

\section{A Lemma on DOUbling Measures}

In this section we follow the philosophy first laid out by Kahane $[\mathrm{K}]$ to construct doubling measures on an interval $I_{0}$. Our measure is the limit of a sequence of measures whose densities are step functions. In Lemma 6.3 we show that mild conditions on these step functions ensure that the limit measure is doubling.

Simple constructions of this type can yield doubling measures which are supported on very small sets. In particular there are examples of such measures which are supported on sets of arbitrarily small, but positive, Hausdorff dimension. Such a measure is equivalent to the derivative of a quasisymmetric mapping that takes a set of small Hausdorff dimension to a set of full one-dimensional Lebesgue measure.

The two interrelated ingredients of this construction are a grid of nested subintervals of $I_{0}$, and a sequence of suitable density functions.

Definition 6.1. A grid of subintervals of an interval $I_{0}$ is a collection $\mathcal{H}=\bigcup_{n=1}^{\infty} \mathcal{H}_{n}$ of subintervals of $I_{0}$ satisfying

1. $\mathcal{H}_{0}=I_{0}$;

2. for each $n \geq 0$, the intervals in $\mathcal{H}_{n}$ have disjoint interiors, and $\left|I_{0} \backslash \bigcup_{I \in \mathcal{H}_{n}}\right|=0$; and

3. for each $n \geq 1$, for each interval $J \in \mathcal{H}_{n}$ there is an interval $I \in \mathcal{H}_{n-1}$ such that $J \subset I$.

The collections $\mathcal{H}_{n}$ are called the layers of intervals in the grid $\mathcal{H}$.

Definition 6.2. A function $F$ defined on a interval $I$ is a step function if it is constant on each interval in some finite or infinite partition of $I$. We say $F$ is $(\delta, \eta)$-suitable for $I$ if $F$ is a step function satisfying

1. $\frac{1}{|I|} \int_{I} F(x) d x=1$;

2. $0<\delta \leq F(x) \leq 1 / \delta$ for all $x \in I$; and

3. $F(x) \equiv 1$ on subintervals $I_{l}$ and $I_{r}$ of $I$ such that $I_{l}$ has the same left endpoint as $I, I_{r}$ has the same right endpoint as $I,\left|I_{l}\right| \geq \eta|I|$, and $\left|I_{r}\right| \geq \eta|I|$.

See Figure 1.

Fix an interval $I_{0}$, and numbers $\delta$ and $\eta$ such that $0<\delta \leq 1$ and $0<\eta \leq 1 / 2$. We construct a sequence $\left\{\mu_{n}\right\}$ of measures on $I_{0}$, whose densities are of the form $d \mu_{n}=F_{n}(x) \cdots F_{1}(x) d x$. We define the functions $F_{n}$ simultaneously with a related grid $\mathcal{H}$ of subintervals of $I_{0}$. Each $F_{n}$ is $(\delta, \eta)$-suitable for each interval from the $(n-1)^{\text {th }}$ layer $\mathcal{H}_{n-1}$ of the grid.

Let $\mu_{0}$ be Lebesgue measure on $I_{0}$. Let $F_{1}$ be any function which is $(\delta, \eta)$-suitable for $I_{0}$. Define $\mu_{1}$ by $d \mu_{1}=F_{1}(x) d x$. Since $F_{1}$ has mean value one on $I_{0}, \mu_{1}$ has the same total mass as $\mu_{0}$. Take any partition of $I_{0}$ into subintervals $J$ such that $F_{1}$ is constant on each $J$. Note that we allow $F_{1}$ to take the same value on adjacent $J$ 's; the $J$ 's need not be maximal. Also, there may be finitely or infinitely many 


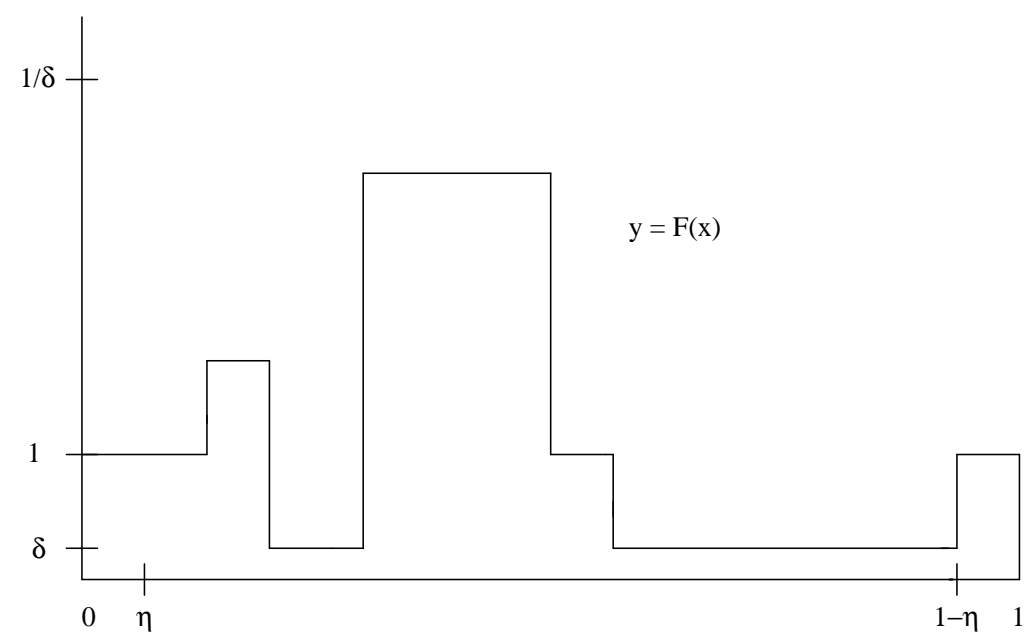

Figure 1. $F(x)$ is $(\delta, \eta)$-suitable for $I=[0,1]$.

$J$ 's. Let $\mathcal{H}_{1}$, the first layer of intervals in the grid, be the collection of subintervals in this partition.

We define the measures $\mu_{n}$ inductively for $n \geq 2$. Suppose we have already defined functions $F_{1}, \ldots, F_{n-1}$, measures $\mu_{1}, \ldots, \mu_{n-1}$, and the layers $\mathcal{H}_{1}, \ldots, \mathcal{H}_{n-1}$ of the grid. In particular, $F_{n-1}$ is constant on each interval $I \in \mathcal{H}_{n-1}$. Let $F_{n}$ be any function which is $(\delta, \eta)$-suitable for each interval $I \in \mathcal{H}_{n-1}$. Define $\mu_{n}$ by

$$
d \mu_{n}=F_{n}(x) d \mu_{n-1}=F_{n}(x) \cdots F_{1}(x) d x .
$$

Again, the fact that $F_{n}$ has mean value one implies that $\mu_{n}$ has the same total mass as $\mu_{0}$. To define the next layer $\mathcal{H}_{n}$ of the grid, we partition each $I \in \mathcal{H}_{n-1}$ into subintervals $J$ such that $F_{n}$ is constant on each $J$. Let $\mathcal{H}_{n}$ be the collection of all these subintervals $J$.

Note that the collection $\mathcal{H}=\bigcup_{n=1}^{\infty} \mathcal{H}_{n}$ of subintervals of $I_{0}$ does form a grid according to the definition above: each interval $J \in \mathcal{H}_{n}$ is contained in some $I \in \mathcal{H}_{n-1}$, and each layer $\mathcal{H}_{n}$ is a partition of $I_{0}$, so $\left|I_{0} \backslash \bigcup_{I \in \mathcal{H}_{n}} I\right|=0$ and the intervals in $\mathcal{H}_{n}$ have disjoint interiors. Also, on each $J \in \mathcal{H}_{n}, \mu_{n}$ is a constant multiple of Lebesgue measure: $d \mu_{n}=\left(\mu_{n}(J) /|J|\right) d x$ on $J$. Furthermore, the total mass assigned to an interval $J \in \mathcal{H}_{n}$ does not change after the $n^{\text {th }}$ step: $\mu_{n}(J)=$ $\mu_{n+1}(J)=\cdots$.

Lemma 6.3. Fix an interval $I_{0}$ and numbers $\delta$ and $\eta$ with $0<\delta \leq 1$ and $0<$ $\eta \leq 1 / 2$. Let $\left\{\mu_{n}\right\}$ be a sequence of measures and $\mathcal{H}$ a grid of subintervals of $I_{0}$ as described above, so that for each $n \geq 1, d \mu_{n}=F_{n}(x) \cdots F_{1}(x) d x$ and $F_{n}(x)$ is $(\delta, \eta)$-suitable for each interval $I \in \mathcal{H}_{n-1}$. Then the sequence $\left\{\mu_{n}\right\}$ converges weakly to a measure $\mu$ on $I_{0}$ which has the same total mass as $\mu_{0}$ and which is doubling with a constant depending only on $\delta$ and $\eta$.

Proof. The $\mu_{n}$ are a sequence of measures, all with the same finite total mass, on a compact interval $I_{0}$. Therefore there is a subsequence converging weakly to a measure $\mu$ on $I_{0}$ with the same mass. It is an exercise to see that this limit measure is unique; in other words the full sequence $\mu_{n}$ converges to $\mu$. The $\mu$-measure of any interval $I \in \mathcal{H}_{n}$ is given by $\mu(I)=\mu_{n}(I)$. 
Let $J$ and $K$ be adjacent intervals, of equal length, contained in $I_{0}$. If, for each $n$, the function $F_{n}$ is constant on $J \cup K$, then $\mu(J)=\mu(K)$. Otherwise, let $m$ be the first integer such that $F_{m}$ is not constant on $J \cup K$. In particular,

$$
\mu_{m}(J) \stackrel{1 / \delta}{\sim} \mu_{m-1}(J)=\mu_{m-1}(K) \stackrel{1 / \delta}{\sim} \mu_{m}(K) .
$$

We show that $\mu(J) \sim \mu_{m}(J)$ and $\mu(K) \sim \mu_{m}(K)$.

We may assume that $F_{m}$ is not constant on $J$. Let $x \in J$ be a point where the value of $F_{m}$ changes. Then for each $n \geq m+1$ there is a neighborhood of $x$ on which $F_{n} \equiv 1$.

Let $J_{r}$ be the part of $J$ to the right of $x$. If $J_{r}$ does not contain any whole interval from $\mathcal{H}$, then $F_{n} \equiv 1$ on $J_{r}$ for all $n \geq m+1$, and so $\mu\left(J_{r}\right)=\mu_{m}\left(J_{r}\right)$. Otherwise, let

$$
\mathcal{L}_{m}=\bigcup_{j}\left\{I_{m, j} \in \mathcal{H}_{m} \mid I_{m, j} \subset J_{r}\right\} .
$$

Then $\mu\left(\mathcal{L}_{m}\right)=\mu_{m}\left(\mathcal{L}_{m}\right)$. (Therefore if $J_{r}$ consists only of whole intervals from $\mathcal{H}_{m}$, in other words if $J_{r}=\mathcal{L}_{m}$, then $\mu\left(J_{r}\right)=\mu_{m}\left(J_{r}\right)$ and we are done.)

The function $F_{m+1}$ is constant on each interval in $\mathcal{H}_{m+1}$. Suppose there is an interval $I_{m+1, j} \in \mathcal{H}_{m+1}$ which meets $J_{r} \backslash \mathcal{L}_{m}$ and on which $F_{m+1} \neq 1$. The interval $I_{m, k} \in \mathcal{H}_{m}$ which contains $I_{m+1, j}$ is adjacent to $\mathcal{L}_{m}$, or has $x$ as its left endpoint in case $\mathcal{L}_{m}$ is empty, and it contains $J_{r} \backslash \mathcal{L}_{m}$. Let $I$ be the largest subinterval of $I_{m, k}$, with the same left endpoint as $I_{m, k}$, on which $F_{m+1} \equiv 1$. $I$ is a union of intervals in $\mathcal{H}_{m+1},|I| \geq \eta\left|I_{m, k}\right|$, and $I \subset J_{r} \backslash \mathcal{L}_{m} \subset I_{m, k}$. Then

$$
\begin{aligned}
\mu\left(J_{r} \backslash \mathcal{L}_{m}\right) & \geq \mu_{m+1}(I) \\
& =\mu_{m}(I) \\
& =\frac{\mu_{m}\left(I_{m, k}\right)}{\left|I_{m, k}\right|}|I| \\
& \geq \eta \mu_{m}\left(I_{m, k}\right) \\
& \geq \eta \mu_{m}\left(J_{r} \backslash \mathcal{L}_{m}\right),
\end{aligned}
$$

and

$$
\begin{aligned}
\mu\left(J_{r} \backslash \mathcal{L}_{m}\right) & \leq \mu_{m}\left(I_{m, k}\right) \\
& \leq \frac{\mu_{m}\left(I_{m, k}\right)}{\left|I_{m, k}\right|} \eta^{-1}|I| \\
& =\eta^{-1} \mu_{m}(I) \\
& \leq \eta^{-1} \mu_{m}\left(J_{r} \backslash \mathcal{L}_{m}\right) .
\end{aligned}
$$

Therefore $\mu\left(J_{r} \backslash \mathcal{L}_{m}\right) \stackrel{1 / \eta}{\sim} \mu_{m}\left(J_{r} \backslash \mathcal{L}_{m}\right)$, and so $\mu\left(J_{r}\right) \stackrel{1 / \eta}{\sim} \mu_{m}\left(J_{r}\right)$.

On the other hand, suppose there is no interval in $\mathcal{H}_{m+1}$ which meets $J_{r} \backslash \mathcal{L}_{m}$ and on which $F_{m+1} \neq 1$. Then $F_{m+1} \equiv 1$ on $J_{r} \backslash \mathcal{L}_{m}$. If $J_{r} \backslash \mathcal{L}_{m}$ contains an interval $I_{m+1, j}$ from $\mathcal{H}_{m+1}$ on which $F_{m+1} \equiv 1$, then the $\mu$-measure of $I_{m+1, j}$ is not only equal to its $\mu_{m+1}$-measure but also to its $\mu_{m}$-measure. Let

$$
\mathcal{L}_{m+1}=\bigcup_{j}\left\{I_{m+1, j} \in \mathcal{H}_{m+1} \mid I_{m+1, j} \subset J_{r} \backslash \mathcal{L}_{m} ; F_{m+1} \equiv 1 \text { on } I_{m+1, j}\right\}
$$

be the union of all such intervals. $\mathcal{L}_{m+1}$ is an interval to the right of the interval $\mathcal{L}_{m}$ and adjacent to it, if both are non-empty. Now $\mu\left(\mathcal{L}_{m+1}\right)=\mu_{m}\left(\mathcal{L}_{m+1}\right)$. If 
$J_{r}=\mathcal{L}_{m} \cup \mathcal{L}_{m+1}$, then $\mu\left(J_{r}\right)=\mu_{m}\left(J_{r}\right)$ and we are done. Otherwise, we have reduced the problem to estimating the $\mu$-measure of $J_{r} \backslash\left(\mathcal{L}_{m} \cup \mathcal{L}_{m+1}\right)$. by

Apply this argument to $J_{r} \backslash \bigcup_{n=m}^{m+i} \mathcal{L}_{n}$ for $i \geq 1$, where $\mathcal{L}_{m+i}$ is defined inductively

$$
\mathcal{L}_{m+i}=\bigcup_{j}\left\{I_{m+i, j} \in \mathcal{H}_{m+i} \mid I_{m+i, j} \subset J_{r} \backslash\left(\bigcup_{n=m}^{m+i-1} \mathcal{L}_{n}\right) ; F_{m+i} \equiv 1 \text { on } I_{m+i, j}\right\} .
$$

Suppose that for some integer $i \geq 1$ there is an interval $I_{m+i+1, j} \in \mathcal{H}_{m+i+1}$ which meets $J_{r} \backslash \bigcup_{n=m}^{m+i} \mathcal{L}_{n}$ and on which $F_{m+i+1} \neq 1$. Let $l$ be the first such integer. As in the case $l=1$ above, let $I_{m+l, k}$ be the interval from $\mathcal{H}_{m+l}$ which contains $I_{m+l+1, j}$ and let $I$ be the largest subinterval of $I_{m+l, k}$ on which $F_{m+l+1} \equiv 1$. Then $I \subset J_{r} \backslash \bigcup_{n=m}^{m+l} \mathcal{L}_{n} \subset I_{m+l, k}$ and so, by the analogues of (6.4) and (6.5), $\mu\left(J_{r}\right) \stackrel{1 / \eta}{\sim} \mu_{m}\left(J_{r}\right)$, and we are done.

On the other hand, suppose there is no such integer $l \geq 1$. If the intervals $\mathcal{L}_{m+i}$, $i \geq 1$, exhaust $J_{r}$, then $\mu\left(J_{r}\right)=\mu_{m}\left(J_{r}\right)$, and we are done. Otherwise, $J_{r} \backslash \bigcup_{n=m}^{\infty} \mathcal{L}_{n}$ is a non-empty interval which has the same right endpoint as $J_{r}$ and which contains no whole interval from $\mathcal{H}$. So $F_{m+i} \equiv 1$ on $J_{r} \backslash \bigcup_{n=m}^{\infty} \mathcal{L}_{n}$ for all $i \geq 1$. Therefore $\mu\left(J_{r} \backslash \bigcup_{n=m}^{\infty} \mathcal{L}_{n}\right)=\mu_{m}\left(J_{r} \backslash \bigcup_{n=m}^{\infty} \mathcal{L}_{n}\right)$, and so $\mu\left(J_{r}\right)=\mu_{m}\left(J_{r}\right)$. This is the last possible case; we have shown that $\mu\left(J_{r}\right) \stackrel{1 / \eta}{\sim} \mu_{m}\left(J_{r}\right)$ in all cases.

The same argument shows that $\mu\left(J_{l}\right) \stackrel{1 / \eta}{\sim} \mu_{m}\left(J_{l}\right)$, where $J_{l}$ is the part of $J$ to the left of $x$. Therefore $\mu(J) \stackrel{1 / \eta}{\sim} \mu_{m}(J)$.

If the function $F_{m}$ is not constant on the adjacent interval $K$, then $\mu(K) \stackrel{1 / \eta}{\sim}$ $\mu_{m}(K)$ by the argument above. Also, if $F_{n} \equiv 1$ on $K$ for all $n \geq m+1$, then $\mu(K) \stackrel{1 / \eta}{\sim} \mu_{m}(K)$. Otherwise, either $F_{n}$ is constant (although not necessarily $\equiv 1$ ) on $K$ for each $n \geq m+1$, or we let $l \geq m+1$ be the first integer such that $F_{l}$ is not constant on $K$. In these cases it is enough to estimate the number of integers $n \geq m+1$ for which $F_{n} \equiv$ constant $\neq 1$ on $K$. We show that this number is bounded by a constant $c=c(\eta)$. Then in the first case $\mu(K) \stackrel{c^{\prime}}{\sim} \mu_{m}(K)$, and in the second $\mu(K) \stackrel{1 / \eta}{\sim} \mu_{l}(K) \stackrel{c^{\prime}}{\sim} \mu_{m}(K)$, where the constants $c^{\prime}$ depend on $c$ and on $\delta$.

Suppose there are at least $k$ integers $n_{1}<\cdots<n_{k}$ greater than $m$ for which $F_{n_{j}} \equiv$ constant $\neq 1$ on the interval $K$. We may assume that $K$ is to the right of $J$. For each $j$ with $1 \leq j \leq k$, there is an interval $I_{j} \in \mathcal{H}_{n_{j}}$ which contains $K$ and whose left endpoint lies in $J$. These intervals are nested: $I_{1} \supset \cdots \supset I_{k}$. Let $L_{j}$ (respectively $R_{j}$ ) be the largest subinterval of $I_{j}$, with the same left (respectively right) endpoint as $I_{j}$, on which $F_{n_{j}} \equiv 1$, and let $M_{j}=I_{j} \backslash\left(L_{j} \cup R_{j}\right)$. Then $\left|M_{j}\right| \leq(1-2 \eta)\left|I_{j}\right|$, and $I_{j} \subset M_{j-1}$ for $2 \leq j \leq k$. See Figure 2 .

Also, $L_{1} \subset J$ and $\eta\left|I_{1}\right| \leq\left|L_{1}\right| \leq|J|$. So

$$
|K| \leq\left|M_{k}\right| \leq(1-2 \eta)\left|I_{k}\right| \leq(1-2 \eta)\left|M_{k-1}\right| .
$$

After $k$ steps we see that

$$
|K| \leq(1-2 \eta)^{k}\left|I_{1}\right| \leq(1-2 \eta)^{k} \eta^{-1}|J|=(1-2 \eta)^{k} \eta^{-1}|K| .
$$

Therefore $k \leq \log \eta / \log (1-2 \eta)$. 


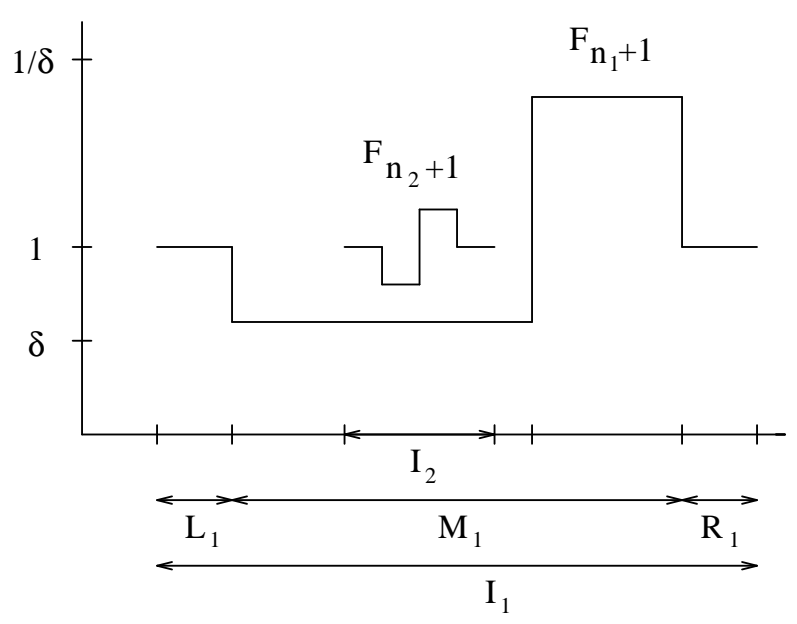

FiguRE 2. Graphs of $F_{n_{1}+1}(x)$ and $F_{n_{2}+1}(x)$

We have shown that $\mu(J) \stackrel{c}{\sim} \mu(K)$, where $c$ depends only on $\delta$ and $\eta$, for every pair $J, K$ of adjacent intervals of equal length. In other words $\mu$ is a doubling measure, and the doubling constant depends only on $\delta$ and $\eta$.

\section{Fundamental DOMAins FOR $\Omega$}

We begin the construction of the doubling measure by describing the fundamental domains for $\Omega=\overline{\mathbf{C}} \backslash K$, where $K \subset[0,1]$ is the classical ternary Cantor set. $G$ is the Fuchsian group such that $\mathbb{D} / G$ is conformally equivalent to $\Omega$. $G$ acts as the covering group of $\Omega$ on the universal covering space $\mathbb{D}$ of $\Omega$. Let $\pi: \mathbb{D} \rightarrow \Omega$ be the covering map; it is a many-to-one conformal mapping which takes each $G$-orbit in $\mathbb{D}$ to a single point in $\Omega$. Normalize $\pi$, by composition with a Möbius transformation of the disc, so that $\pi(0)=\infty$.

A fundamental domain for $\Omega$ is a domain $\mathcal{F}$ in $\mathbb{D}$ such that $\overline{\mathcal{F}}$ contains at least one point from every $G$-orbit, and $\mathcal{F}$ contains no two $G$-equivalent points. The domain $\Omega=\overline{\mathbf{C}} \backslash K$ is a Denjoy domain; that is, it is the complement in $\overline{\mathbf{C}}$ of a closed linear set. Denjoy domains have fundamental domains which have a particularly simple form; namely, they are orthocircular. This means that the boundary is of the form $\partial \mathcal{F}=E \cup \bigcup_{n=1}^{\infty} A_{n}$, where $E$ is a closed subset of the unit circle $\partial \mathbb{D}$, and the $A_{n}$ 's (called orthocircular arcs) are disjoint arcs of circles which meet $\partial \mathbb{D}$ at right angles. The covering map takes the set $\partial \mathcal{F} \cap \partial \mathbb{D}$ to $\partial \Omega$, and it takes the orthocircular arcs comprising $\partial \mathcal{F} \backslash \partial \mathbb{D}$ to the components of $\overline{\mathbb{R}} \backslash \partial \Omega$. The images $\{g(\mathcal{F})\}_{g \in G}$ are orthocircular domains which tile the disc. (See [RR]; they consider the case where the boundary $\partial \Omega$ has positive length, but a Denjoy domain $\Omega$ with $|\partial \Omega|=0$ also has an orthocircular fundamental domain $\mathcal{F}$, with $|\partial \mathcal{F} \cap \partial \mathbb{D}|=0$.)

We refer to the images in the disc of the upper and lower half planes under the branches of $\pi^{-1}$ as half fundamental domains for $\Omega=\overline{\mathbf{C}} \backslash K$. Each half fundamental domain $D$ is orthocircular. To fix ideas, suppose that $\pi(D)$ is the upper half plane. The boundary $\overline{\mathbb{R}}$ of the upper half plane lifts via $\pi^{-1}$ to the boundary of $D$. The Cantor set $K$ lifts to the closed subset $\partial D \cap \partial \mathbb{D}$ of the circle, and the open intervals 
which are the components of $\overline{\mathbb{R}} \backslash K$ lift to the orthocircular arcs in the boundary of $D$.

We now fix a tiling of the disc by half fundamental domains for $\Omega$, together with one fundamental domain for $\Omega$. Since $\pi(0)=\infty$, one of the preimages under $\pi$ of the open interval $\overline{\mathbb{R}} \backslash[0,1]$ is a diameter $\gamma$ of $\mathbb{D}$. Let $D$ be the preimage of the upper half plane whose boundary contains $\gamma$. Let $D^{\prime}$ be the reflection of $D$ in $\gamma$. $D^{\prime}$ is a preimage of the lower half plane. Then $D \cup \gamma \cup D^{\prime}$ is a fundamental domain for $\Omega$; it contains the origin; and it is symmetric with respect to the diameter $\gamma$. We denote this fundamental domain by $\mathcal{F}_{0}$; it is, by symmetry, the normal fundamental domain for $\Omega$. Its image under the covering map is $\pi\left(\mathcal{F}_{0}\right)=\overline{\mathbf{C}} \backslash[0,1]$. Each open interval in $[0,1] \backslash K$ lifts to two orthocircular arcs in $\partial \mathcal{F}_{0}$.

Tile the remainder of the disc, $\mathbb{D} \backslash \mathcal{F}_{0}$, by the half fundamental domains which are the $G$-images of $D$ and $D^{\prime}$. These half fundamental domains are indexed in a natural way by the number of orthocircular boundary arcs separating them from the origin. Write $\Omega_{1, j}$ for any half fundamental domain which is adjacent to $\mathcal{F}_{0}$; that is, which is separated from the origin 0 by a single orthocircular arc. Similarly, write $\Omega_{n, j}$ for a half fundamental domain which is separated from 0 by $n$ orthocircular arcs in the boundaries of the half fundamental domains in the fixed tiling of $\mathbb{D}$.

A note on orientation: we think of the direction from a point in the disc towards the unit circle as down, and of the direction towards the origin as up. Terms like below and above are to be understood in the same way; below means closer to the unit circle. Given a half fundamental domain $\Omega_{n, j}$, we refer to the large orthocircular arc, denoted $A_{n, j}$, which separates $\Omega_{n, j}$ from 0 as the upper part of the boundary of $\Omega_{n, j}$, and to $\partial \Omega_{n} \backslash A_{n, j}$ as the lower part of the boundary of $\Omega_{n, j}$.

Let $\mathcal{A}_{1}=\partial \mathcal{F}_{0} \backslash \partial \mathbb{D}$ be the union of the orthocircular arcs in the boundary of $\mathcal{F}_{0}$. Each arc $A_{1, j} \subset \mathcal{A}_{1}$ is the upper part of some $\partial \Omega_{1, j}$. For each $n \geq 2$, let $\mathcal{A}_{n}$ be the union, over all $j$, of the orthocircular arcs in the lower parts of the boundaries of the $\Omega_{n-1, j}$ :

$$
\mathcal{A}_{n}=\left[\bigcup_{j} \partial \Omega_{n-1, j} \backslash \partial \mathbb{D}\right] \backslash \mathcal{A}_{n-1}
$$

The union $\mathcal{A}=\bigcup_{n=1}^{\infty} \mathcal{A}_{n}$ is the collection of all orthocircular boundary arcs which appear in the tiling of $\mathbb{D} \backslash \mathcal{F}_{0}$ by half fundamental domains.

We show in Section 10 that the orthocircular boundary arcs are uniformly hyperbolically separated: the hyperbolic distance between any two of these arcs is greater than a fixed positive constant. It follows from this observation that the boundaries of the half fundamental domains are chord-arc curves, with a uniform chord-arc constant. We will not need this fact, but we will need to show (Sections 13 and 14) that certain subsets of the half fundamental domains are chord-arc with uniform chord-arc constants.

\section{WHITNEY DECOMPOSITIONS}

In this section we make a Whitney decomposition of the components of $\overline{\mathbb{R}} \backslash K$, and show that it lifts via $\pi^{-1}$ to a Whitney decomposition of the orthocircular arcs in the boundaries of the half fundamental domains in $\mathbb{D}$. The intervals in this lifted decomposition are the vertices in the tree described in Section 4.

The Cantor set $K$ is formed by removing the open middle third $\left(\frac{1}{3}, \frac{2}{3}\right)$ from $[0,1]$, then removing the open middle thirds $\left(\frac{1}{9}, \frac{2}{9}\right)$ and $\left(\frac{7}{9}, \frac{8}{9}\right)$ from the closed 
intervals $\left[0, \frac{1}{3}\right]$ and $\left[\frac{2}{3}, 1\right]$ which remain, and so on. We refer to the closed intervals $[0,1],\left[0, \frac{1}{3}\right],\left[\frac{2}{3}, 1\right],\left[0, \frac{1}{9}\right],\left[\frac{2}{9}, \frac{1}{3}\right], \ldots$ which appear in this procedure as closed construction intervals of $K$.

A Whitney decomposition of an open interval $L$ in $\mathbb{R}$ is a partition of $L$ into closed intervals $J$, with disjoint interiors, such that the Euclidean length of each $J$ is comparable to the Euclidean distance from $J$ to the nearest endpoint of $L$. In other words, there is a constant $c>0$ such that $|J| \stackrel{c}{\sim} \operatorname{dist}(J, \mathbb{R} \backslash L)$ for each $J$. This also implies that for each point $x$ in such an interval $J$, the distance from $x$ to $\mathbb{R} \backslash L$ is comparable to the distance from $J$ to $\mathbb{R} \backslash L$. The intervals $J$ in a Whitney decomposition are called Whitney intervals. We will also deal with Whitney decompositions of orthocircular arcs; these are defined analogously, so that the length of each Whitney interval in the arc is comparable to its distance from the nearest end of the arc.

Write $\overline{\mathbb{R}} \backslash K$ as a countable union of disjoint open intervals; let $L$ denote any one of these intervals. We now fix a Whitney decomposition of each component $L$ of $\overline{\mathbb{R}} \backslash K$.

First consider a component $L=(a, b)$ of $[0,1] \backslash K . \quad L$ is an open interval of length $3^{-k}$, for some $k \geq 1$. Partition $L$ into subintervals of length $3^{-n-k}, n \geq 1$, as follows. For $n \geq 1$, let

$$
\begin{aligned}
J_{n} & =\left[b-3^{-n+1} \frac{|L|}{2}, b-3^{-n} \frac{|L|}{2}\right], \text { and } \\
J_{-n} & =\left[a+3^{-n} \frac{|L|}{2}, a+3^{-n+1} \frac{|L|}{2}\right] .
\end{aligned}
$$

Then $J_{1}$ is the interval whose left endpoint is the midpoint of $L$ and which extends two-thirds of the way towards the right endpoint of $L$; for $n \geq 2, J_{n}$ is the interval whose left endpoint is the right endpoint of $J_{n-1}$ and which extends two-thirds of the way towards the right endpoint of $L$; and for $n \geq 1, J_{-n}$ is the reflection of $J_{n}$ in the midpoint of $L$. The intervals $\left\{J_{ \pm n}\right\}_{n \geq 1}$ form a Whitney decomposition of $L$, $\left|J_{ \pm n}\right|=2 \operatorname{dist}\left(J_{ \pm n}, K\right)$, and $\left|J_{ \pm n}\right|=3^{-n}|L|=3^{-n-k}$.

Let $N$ be a large integer; its value will be fixed below. It is convenient to amalgamate the $2 N$ intervals $J_{-N}, \ldots, J_{N}$ which are closest to the midpoint of $L$ into a single interval: Let

$$
J_{c}=J_{-N} \cup \cdots \cup J_{N}
$$

This interval has length $\left|J_{c}\right|=\left(1-3^{-N}\right)|L|$, and its distance from $K$ is $2^{-1} \cdot 3^{-N}|L|$, so it satisfies $\left|J_{c}\right|=2\left(3^{N}-1\right) \operatorname{dist}\left(J_{c}, K\right)$.

The intervals $\left\{J_{ \pm n}\right\}_{n \geq N+1}$ and $J_{c}$ form a Whitney decomposition of $L$, with constant $2\left(3^{N}-1\right)$.

Now consider the remaining component $L=\overline{\mathbb{R}} \backslash[0,1]$. Fix the small number $\sigma=\left(2 \cdot 3^{N+2}\right)^{-1}$; the reasons for this particular choice will become apparent later. Let

$$
J_{\infty}=\overline{\mathbb{R}} \backslash(-\sigma, 1+\sigma)
$$


$J_{\infty}$ is an interval of large but finite hyperbolic length, which contains the point at infinity. For $n \geq N+3$, let

$$
\begin{aligned}
J_{n} & =\left[-\frac{1}{2} \frac{1}{3^{n-1}},-\frac{1}{2} \frac{1}{3^{n}}\right], \text { and } \\
J_{-n} & =\left[1+\frac{1}{2} \frac{1}{3^{n}}, 1+\frac{1}{2} \frac{1}{3^{n-1}}\right] .
\end{aligned}
$$

Then $J_{N+3}=[-\sigma,-\sigma / 3]$, and for $n \geq N+4, J_{n}$ is the interval whose left endpoint is the right endpoint of $J_{n-1}$ and which extends two-thirds of the way towards 0 . For $n \geq N+3, J_{-n}$ is the reflection of $J_{n}$ through the midpoint of $[0,1]$. The intervals $\left\{J_{ \pm n}\right\}_{n \geq N+3}$ form a Whitney decomposition of $[-\sigma, 0) \cup(1,1+\sigma]$, in which $\left|J_{ \pm n}\right|=3^{-n}=2 \operatorname{dist}\left(J_{ \pm n}, K\right)$.

Make the convention that

$$
\left|J_{\infty}\right|=1 / 3 .
$$

With this convention, the intervals $\left\{J_{ \pm n}\right\}_{n \geq N+3}$ and $J_{\infty}$ form a Whitney decomposition of $L=\overline{\mathbb{R}} \backslash[0,1]$, with constant $2 \cdot 3^{N+3}$.

We use the phrase the Whitney decomposition of $\overline{\mathbb{R}} \backslash K$ to denote the collection of Whitney intervals $\left\{J_{ \pm n}\right\}_{n \geq N+1}$ and $J_{c}$ in the components of $[0,1] \backslash K$, together with the Whitney intervals $\left\{J_{ \pm n}\right\}_{n \geq N+3}$ and $J_{\infty}$ in $\overline{\mathbb{R}} \backslash[0,1]$. This decomposition will remain fixed for the rest of the construction. We call the intervals $\left\{J_{ \pm n}\right\}$ standard and the intervals $J_{c}$ and $J_{\infty}$ non-standard.

The inverse $\pi^{-1}$ of the covering map lifts the components of $\overline{\mathbb{R}} \backslash K$ to the orthocircular arcs $\bigcup_{n} \mathcal{A}_{n}$ which appear in the boundaries of the half fundamental domains in our tiling of $\mathbb{D}$. $\pi^{-1}$ lifts the Whitney decomposition of $\overline{\mathbb{R}} \backslash K$ to a decomposition of $\bigcup_{n} \mathcal{A}_{n}$ into closed intervals with disjoint interiors.

The main result of this section is that this is a Euclidean Whitney decomposition of the orthocircular $\operatorname{arcs} \bigcup_{n} \mathcal{A}_{n}$.

Lemma 8.1. The Whitney decomposition of $\overline{\mathbb{R}} \backslash K$ lifts via $\pi^{-1}$ to a Whitney decomposition of the orthocircular arcs $\bigcup_{n} \mathcal{A}_{n}$.

Proof. Since $\partial \Omega=K$ is uniformly perfect, there is a constant $c_{\Omega}>0$ such that the function $\lambda_{\Omega}$ satisfies

$$
\frac{c_{\Omega}}{\operatorname{dist}(w, K)} \leq \lambda_{\Omega}(w) \leq \frac{2}{\operatorname{dist}(w, K)}
$$

for all $w$ in $\Omega$, where the element of hyperbolic arclength in $\Omega$ is $d s=\lambda_{\Omega}(w)|d w|$. Combined with the relation $|J|=2 \operatorname{dist}(J, K)$ for standard Whitney intervals $J$, this implies that $\ell_{\text {hyp }}(J) \sim 1$ with a uniform constant for all standard $J$ in $\overline{\mathbb{R}} \backslash K$. Similarly, since the ratio $\left|J_{c}\right| / \operatorname{dist}\left(J_{c}, K\right)$ is the same for all non-standard intervals $J_{c}, \ell_{\text {hyp }}\left(J_{c}\right) \sim 1$ for all such $J_{c}$. Also, the hyperbolic length of $J_{\infty}$ depends only on the constant $\sigma$.

These observations imply that the intervals $I$ in the preimage of the Whitney decomposition of $\overline{\mathbb{R}} \backslash K$ are all of comparable hyperbolic length, because the covering map $\pi$ preserves hyperbolic length. So $1 / r \leq \ell_{\text {hyp }}(I) \leq r$ for all $I$, where the constant $r$ depends on the uniformly perfect constant of $K$, on the constant of the Whitney decomposition of $\overline{\mathbb{R}} \backslash K$, and on the choice of $\sigma$ in the definition of $J_{\infty}$. 
It follows that, for each $I$,

$$
\frac{1}{2 r}\left(1-\left|z_{1}\right|\right) \leq|I| \leq 2 r e^{r}\left(1-\left|z_{1}\right|\right)
$$

where $z_{1}$ is the endpoint of $I$ closest to $\partial \mathbb{D}$. Therefore the intervals $I$ form a Whitney decomposition of the orthocircular $\operatorname{arcs} \bigcup_{n} \mathcal{A}_{n}$.

\section{Construction of Palm leaves AND the GRID of intervals}

In this section we construct a grid $\mathcal{H}$ of subintervals of the unit circle, of the kind described in Section 6. We already have a collection of Whitney intervals which form a Whitney decomposition of the orthocircular arcs $\bigcup_{n} \mathcal{A}_{n}$ in the boundaries of the half fundamental domains in the tiling of the unit disc. The idea is to project these Whitney intervals onto the unit circle, using a projection map $P$ defined below. The projections of the intervals in $\mathcal{A}_{n}$ form the $n^{\text {th }}$ layer $\mathcal{H}_{n}$ of the grid. Heuristically, the projection $P$ is almost radial projection. Let ${ }^{\wedge}$ denote the inverse of the projection $P$ : given a grid interval $I, \widehat{I}$ denotes the unique Whitney interval in $\bigcup_{n} \mathcal{A}_{n}$ such that $P(\widehat{I})=I$.

Recall that $\mathcal{A}_{1}=\partial \mathcal{F}_{0} \backslash \partial \mathbb{D}$ is the collection of orthocircular arcs in the boundary of the fundamental domain $\mathcal{F}_{0}$ which contains the origin; and that for $n \geq 2, \mathcal{A}_{n}$ consists of the collection of orthocircular boundary arcs, from the tiling of $\mathbb{D} \backslash \mathcal{F}_{0}$ by half fundamental domains, which are separated from 0 by exactly $n-1$ other orthocircular boundary arcs.

In order to define the projection $P$, we make a construction in $\Omega=\overline{\mathbf{C}} \backslash K$ and lift it to the disc via $\pi^{-1}$. An outline follows. Given any Whitney interval $\widehat{I} \subset \mathcal{A}_{n}$, we must specify which of the intervals $\widehat{I}_{n+1, k} \subset \mathcal{A}_{n+1}$ should project via $P$ to subintervals of $P(\widehat{I})$. (In terms of the tree described in Section 4, whose vertices are the Whitney intervals in $\bigcup_{n} \mathcal{A}_{n}$, we are now specifying the adjacencies between vertices; in other words which pairs of vertices are connected by edges.) Let $A_{n, j}$ be the orthocircular arc containing $\widehat{I}$, and let $\Omega_{n, j}$ be the half fundamental domain below $A_{n, j}$. The covering map $\pi$ maps $\widehat{I}$ to a Whitney interval $J$ in $\overline{\mathbb{R}} \backslash K$; it maps $A_{n, j}$ to the component $L$ of $\overline{\mathbb{R}} \backslash K$ which contains $J=\pi(\widehat{I})$; it maps $\Omega_{n, j}$ to either the upper or lower half plane; and it maps $\partial \Omega_{n, j}$ to $\overline{\mathbb{R}}$. See Figure 3.

To each interval $J$ in the Whitney decomposition of $L$ we associate an interval $E_{J} \subset \overline{\mathbb{R}} \backslash L$, in such a way that the $E_{J}$ for all $J$ in $L$ have disjoint interiors and their union is $\overline{\mathbb{R}} \backslash L$. (The rest of this section contains precise definitions of these intervals $E_{J}$.) These intervals $E_{J}$ are of a certain form; in particular, for most $J$ the Euclidean length $\left|E_{J}\right|$ is a large fixed multiple of $|J|$, and the endpoints of $E_{J}$ always lie in the Cantor set $K$.

Let $\widetilde{I}$ be the segment of $\partial \Omega_{n, j}$ such that $\pi(\widetilde{I})=E_{J} \cdot \widetilde{I}$ lies in the lower part of the boundary of $\Omega_{n, j}$, and its endpoints lie in the unit circle. Let $P(\widehat{I})$ be the arc of the unit circle which has the same endpoints as $\widetilde{I}$ and which lies below $\widetilde{I}$. With this definition the intervals $\widehat{I}_{n+1, k} \subset \mathcal{A}_{n+1}$ whose projections $P\left(\widehat{I}_{n+1, k}\right)$ are contained in $P(\widehat{I})$ are precisely those intervals $\widehat{I}_{n+1, k}$ which are contained in $\widetilde{I}$.

The projections $\left\{P\left(\widehat{I}_{n, j}\right)\right\}_{j}$ of the Whitney intervals $\widehat{I}_{n, j}$ in $\mathcal{A}_{n}$ constitute the $n^{\text {th }}$ layer $\mathcal{H}_{n}$ of the grid of subintervals of the circle. With the definition of $P$ outlined above, each interval $P\left(\widehat{I}_{n+1, k}\right)$ in $\mathcal{H}_{n+1}$ is contained in some $P\left(\widehat{I}_{n, j}\right)$ from the previous layer $\mathcal{H}_{n}$. Also, since the intervals $E_{J}$ have pairwise disjoint interiors 

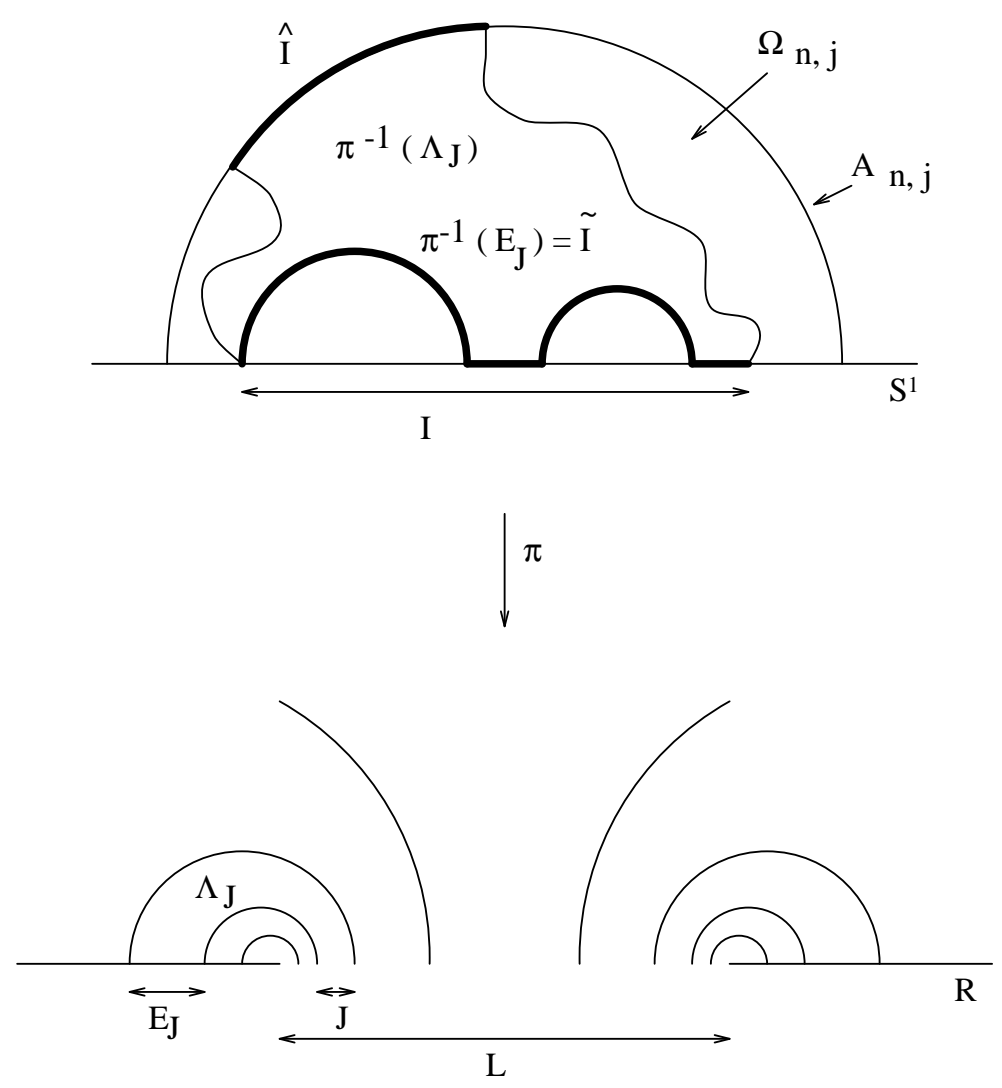

FiguRE 3. Fan of palm leaves $\Lambda_{J}$ based at the Whitney intervals $J=\pi(\widehat{I})$ in $L$; and a preimage in $\mathbb{D}$ of one leaf $\Lambda_{J}$

for all $J$ in each $L$, the $P\left(\widehat{I}_{n, j}\right)$ at the $n^{\text {th }}$ level have pairwise disjoint interiors. Finally, by the remarks in Section 5 above, the part of $\partial \mathbb{D}$ below the orthocircular $\operatorname{arcs}$ in $\mathcal{A}_{n}$ has full Lebesgue measure in $\partial \mathbb{D}$ for each $n \geq 1$, so $\bigcup_{\widehat{I} \subset \mathcal{A}_{n}} P(\widehat{I})$ has full Lebesgue measure in $\partial \mathbb{D}$ for each $n \geq 1$. In other words, the union of the intervals $P(\widehat{I})$ in $\mathcal{H}_{n}$ covers $\partial \mathbb{D}$, up to a set of measure zero. Thus the layers $\mathcal{H}_{n}$ of intervals form a grid $\mathcal{H}$ of subintervals of $\partial \mathbb{D}$, according to the definition in Section 6 .

If $\widehat{R}$ is a segment of the boundary of some half fundamental domain $\Omega_{n, j}$, consisting of a collection of whole Whitney intervals $\left\{\widehat{I}_{n+1, k}\right\}_{k}$, possibly together with a subset $E$ of $\partial \Omega_{n, j} \cap \partial \mathbb{D}$, then we define $P(\widehat{R})$ to be $E \cup \bigcup_{k} P\left(\widehat{I}_{n+1, k}\right)$.

We now give the details of the construction of the grid $\mathcal{H}$ of intervals, in particular defining the intervals $E_{J}$ associated to the Whitney intervals $J$ in $\overline{\mathbb{R}} \backslash K$. Let $N \gg 1$ be the same large integer as in the previous section. Until the last part of the paper we regard $N$ as fixed, and we make all our geometric constructions using this fixed value.

Let $L$ be a component of $\overline{\mathbb{R}} \backslash K$. We define a decomposition of the upper half plane into infinitely many regions $\Lambda_{J}$, indexed by the Whitney intervals $J$ in $L$. See Figure 3. We refer to these regions as palm leaves based at the Whitney intervals $J$ in $L$, and to the collection of these regions as a fan of palm leaves, based at $L$. The 


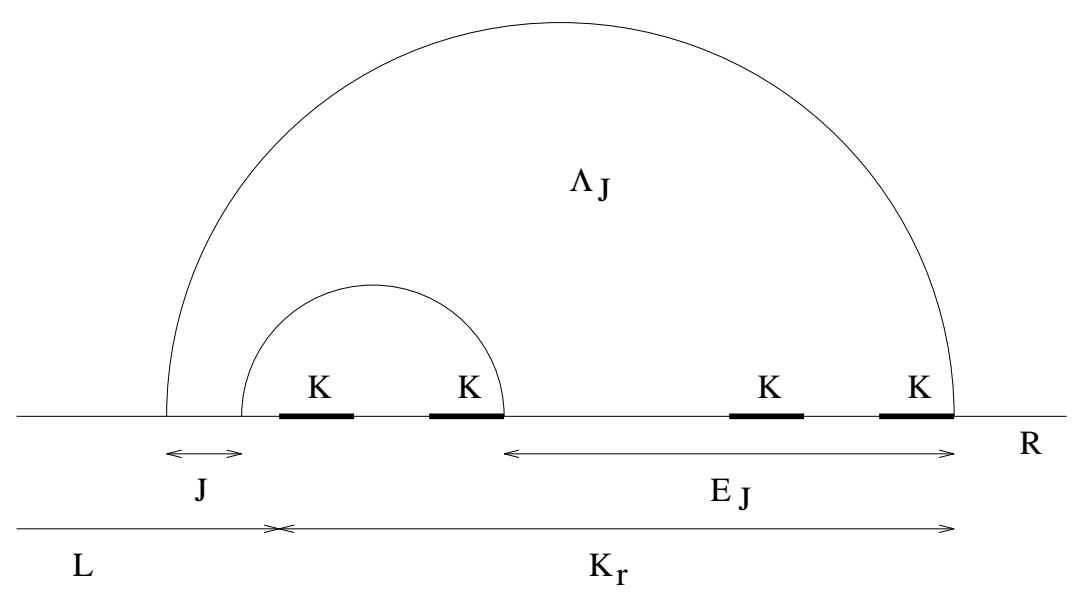

Figure 4. Standard palm leaf $\Lambda_{J}$ for $J=J_{N+1}$. Not to scale: $\left|E_{J}\right|=2 \cdot 3^{N}|J|$ and $N \gg 1$.

boundary of the leaf $\Lambda_{J}$ based at the Whitney interval $J$ consists of two intervals in $\overline{\mathbb{R}}, J$ itself and $E_{J}$, together with two non-intersecting semicircles which join the endpoints of $J$ to the endpoints of $E_{J}$ and which meet $\mathbb{R}$ at right angles. We call $J$ the base and $E_{J}$ the tip of the leaf $\Lambda_{J}$. The union over all $J$ in $L$ of the intervals $E_{J}$ covers $\overline{\mathbb{R}} \backslash L$.

Once the entire construction of the fans of palm leaves is complete, we reflect them in the real axis to obtain analogous fans in the lower half plane.

We distinguish two cases: when the interval $L$ is a component of $[0,1] \backslash K$, and when $L=\overline{\mathbb{R}} \backslash[0,1]$.

Case 1. Let $L$ be a component of $\overline{\mathbb{R}} \backslash K$ which lies in [0,1] and has length $|L|=$ $3^{-l}$ for some integer $l \geq 1$. Write $L=\left(a, a+3^{-l}\right)$. Let $K_{l}=\left[a-3^{-l}, a\right]$ and $K_{r}=\left[a+3^{-l}, a+2 \cdot 3^{-l}\right]$ be the closed construction intervals of $K$ of length $\left|K_{l}\right|=\left|K_{r}\right|=|L|=3^{-l}$ such that $K_{l}$ is immediately to the left of $L$ and $K_{r}$ is immediately to the right of $L$. The Whitney intervals in $L$ are enumerated from left to right as $\ldots, J_{-N-2}, J_{-N-1}, J_{c}, J_{N+1}, J_{N+2}, \ldots$. Recall that $\left|J_{ \pm n}\right|=3^{-n}|L|$, for $n \geq N+1$, and $\left|J_{c}\right|=\left(1-3^{-N}\right)|L|$.

We refer to the Whitney intervals $J_{ \pm n}$ in $L$ such that $n \geq N+1$ as standard intervals. For each standard interval $J$ we define a leaf $\Lambda_{J}$ based at $J$ such that the tip $E_{J}$ of the leaf has length $\left|E_{J}\right|=2 \cdot 3^{N}|J|$. We begin with the standard Whitney interval $J_{N+1}$, which has length $\left|J_{N+1}\right|=3^{-N-1}|L|$ and is in the right half of $L$.

Let $E_{J_{N+1}}$ be the closed interval which has the same right endpoint as $K_{r}$ and whose length is two-thirds the length of $K_{r} . E_{J_{N+1}}$ consists of a closed construction interval of $K$ and the closure of an adjacent open interval in [0,1] $\backslash K$ of the same length. Now $\left|E_{J_{N+1}}\right|=\frac{2}{3}\left|K_{r}\right|=\frac{2}{3}|L|=\frac{2}{3} \cdot 3^{N+1}\left|J_{N+1}\right|=2 \cdot 3^{N}\left|J_{N+1}\right|$. Join the left endpoint of $J_{N+1}$ to the right endpoint of $E_{J_{N+1}}$ by a semicircle in the upper half plane. Join the right endpoint of $J_{N+1}$ to the left endpoint of $E_{J_{N+1}}$ in the same way. Let the leaf $\Lambda_{J_{N+1}}$ be the region in the upper half plane bounded by $J_{N+1}, E_{J_{N+1}}$, and the two semicircles. See Figure 4.

Consider the next standard Whitney interval $J_{N+2}$; it has length $3^{-N-2}|L|$ and is immediately to the right of $J_{N+1}$. Construct the leaf $\Lambda_{J_{N+2}}$ and its tip $E_{J_{N+2}}$ 
exactly as for $J_{N+1}$ : Let $E_{J_{N+2}}$ be the right two-thirds of $K_{r} \backslash E_{J_{N+1}}$, and join the endpoints of $J_{N+2}$ to those of $E_{J_{N+2}}$ by semicircles. The leaf $\Lambda_{J_{N+2}}$ bounded by $J_{N+2}, E_{J_{N+2}}$, and these semicircles is a copy of $\Lambda_{J_{N+1}}$, shrunk by a factor of one-third. The smaller semicircle in the boundary of $\Lambda_{J_{N+1}}$ is the larger semicircle in the boundary of $\Lambda_{J_{N+2}}$. Clearly $\left|E_{J_{N+2}}\right|=2 \cdot 3^{N}\left|J_{N+2}\right|$.

Repeat this construction for the intervals $J_{N+k}, k \geq 1$, obtaining a sequence of leaves $\Lambda_{J_{N+k}}$ based at $J_{N+k}$ which satisfy $\left|E_{J_{N+k}}\right|=2 \cdot 3^{N}\left|J_{N+k}\right|$. The leaves $\Lambda_{J_{N+k}}$ are all congruent to each other via dilations by powers of three. The intervals $E_{J_{N+k}}, k \geq 1$, have disjoint interiors and their union is $K_{r}$, the closed construction interval of length $|L|$ to the right of $L$.

Define leaves $\Lambda_{J_{-N-k}}, k \geq 1$, for the standard intervals at the left end of $L$, by reflecting the leaves $\Lambda_{J_{N+k}}$ through the perpendicular bisector of $L$. The leaf $\Lambda_{J_{-N-k}}$ based at $J_{-N-k}$ is the mirror image of the leaf $\Lambda_{J_{N+k}}$ based at $J_{N+k}$. The intervals $E_{J_{-N-k}}, k \geq 1$, cover $K_{l}$, the closed construction interval of length $|L|$ to the left of $L$.

We refer to the leaves we have just constructed for the standard intervals $J_{ \pm(N+k)}$, $k \geq 1$, as standard leaves, and to their tips as standard $E_{J}$ 's. We also refer to any grid interval $I$ such that $J=\pi(\widehat{I})$ is standard as a standard grid interval. For any standard leaf $\Lambda_{J},\left|E_{J}\right|=2 \cdot 3^{N}|J|$; the endpoints of $E_{J}$ lie in the Cantor set $K$; and $E_{J}$ consists of a closed construction interval of length $3^{N}|J|$ together with the closure of an adjacent open interval in $\overline{\mathbb{R}} \backslash K$, also of length $3^{N}|J|$.

It remains to define the leaf for the central interval $J_{c}=J_{-N} \cup \cdots \cup J_{N}$. Let $E_{J_{c}}=\overline{\mathbb{R}} \backslash\left(K_{l} \cup L \cup K_{r}\right)$. Define the leaf $\Lambda_{J_{c}}$ to be the region in the upper half plane bounded by $J_{c}, E_{J_{c}}$, and the two non-intersecting semicircles joining the endpoints of $J_{c}$ to those of $E_{J_{c}}$. The endpoints of $E_{J_{c}}$ lie in the Cantor set $K$. Notice that when $L=\left(\frac{1}{3}, \frac{2}{3}\right), E_{J_{c}}$ is exactly $\overline{\mathbb{R}} \backslash[0,1]$, since the tips of the leaves for the standard intervals in $\left(\frac{1}{3}, \frac{2}{3}\right)$ take up all of $\left[0, \frac{1}{3}\right] \cup\left[\frac{2}{3}, 1\right]$. For smaller intervals $L, E_{J_{c}}$ contains $\overline{\mathbb{R}} \backslash[0,1]$ and part of $[0,1]$. We use the term non-standard to refer to $J_{c}, \Lambda_{J_{c}}, E_{J_{c}}$, and any grid interval $I$ such that $\pi(\widehat{I})=J_{c}$.

To summarize: given an open interval $L$ which is a component of $[0,1] \backslash K$, we have defined a fan of palm leaves $\Lambda_{J}$ based at the Whitney intervals $J$ in $L$, such that:

- the $\Lambda_{J}$ 's tile the upper half plane;

- the tips $E_{J}$ of the leaves are intervals whose union is $\overline{\mathbb{R}} \backslash L$;

- the $E_{J}$ have disjoint interiors;

- the endpoints of each $E_{J}$ lie in the Cantor set $K$;

- $\left|E_{J}\right|=2 \cdot 3^{N}|J|$ for all standard $J$, i.e. for all but the central interval $J_{c}$ in $L$; and

- each standard $E_{J}$ consists of a closed construction interval of $K$ and the closure of an adjacent open interval in $\overline{\mathbb{R}} \backslash K$ of the same length.

Case 2. $L$ is the component $\overline{\mathbb{R}} \backslash[0,1]$ of $\overline{\mathbb{R}} \backslash K$. Then $L$ consists of the large interval $J_{\infty}=\overline{\mathbb{R}} \backslash(-\sigma, 1+\sigma)$ and Whitney intervals $\left\{J_{ \pm n}\right\}_{n \geq N+3}$ in $[-\sigma, 0) \cup(1,1+\sigma]$, where $\sigma=\left(2 \cdot 3^{N+2}\right)^{-1}$.

For the interval $J_{\infty}=\overline{\mathbb{R}} \backslash(-\sigma, 1+\sigma)$, define $E_{J_{\infty}}=\left[\frac{1}{9}, \frac{8}{9}\right]$. Join the endpoints of $J_{\infty}$ to the endpoints of $\left[\frac{1}{9}, \frac{8}{9}\right]$ by non-intersecting semicircles in the upper half plane, and let the leaf $\Lambda_{J_{\infty}}$ be the region in the upper half plane bounded by $J_{\infty}$, $E_{J_{\infty}}$, and the two semicircles. See Figure 5(a). 
$\Lambda_{\mathrm{J}}$

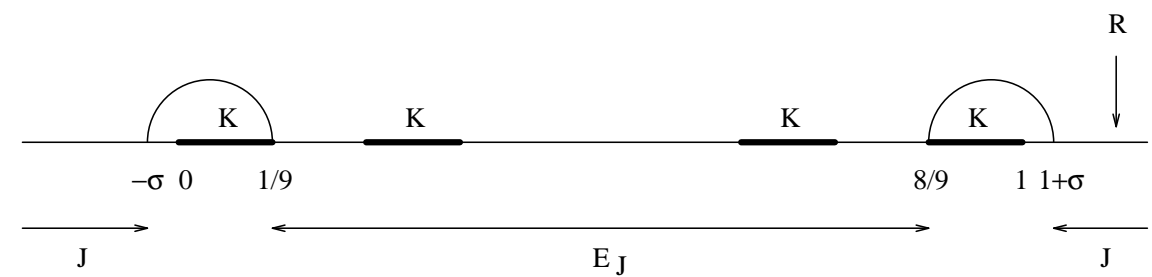

Figure 5(a). Non-standard palm leaf $\Lambda_{J_{\infty}}$ for $J=J_{\infty}$.

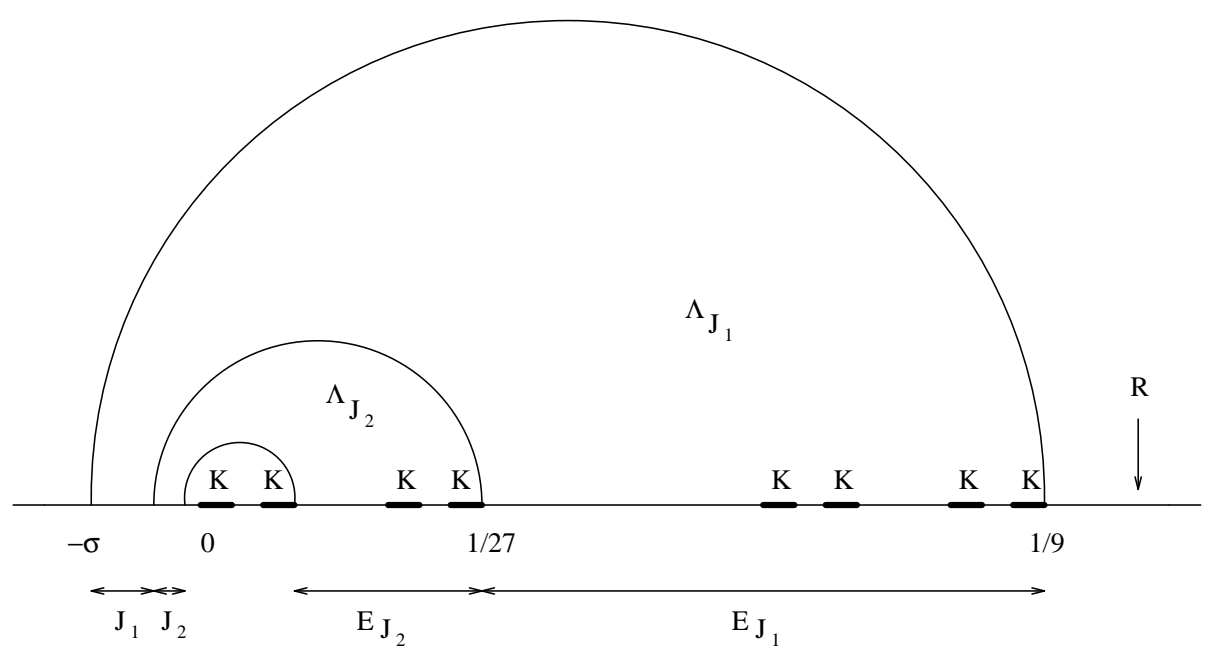

Figure $5(\mathrm{~b})$. Standard palm leaves for $J_{1}=J_{N+3}, J_{2}=J_{N+4}$ in $[-\sigma, 0)$.

The Whitney intervals in $[-\sigma, 0)$ are enumerated from left to right as $J_{N+3}$, $J_{N+4}, \ldots$, in order of decreasing size. See Figure 5(b). For $J_{N+3}=[-\sigma,-\sigma / 3]$, let $E_{J_{N+3}}=\left[3^{-3}, 3^{-2}\right]$. Form the leaf $\Lambda_{J_{N+3}}$ by joining the endpoints of $J_{N+3}$ to those of $E_{J_{N+3}}$ by two non-intersecting semicircles which meet $\mathbb{R}$ at right angles. Then $\left|J_{N+3}\right|=\frac{2}{3} \sigma=3^{-(N+3)}=\left(2 \cdot 3^{N}\right)^{-1}\left|E_{J_{N+3}}\right|$. So $\Lambda_{J_{N+3}}$ and $E_{J_{N+3}}$ are of the standard form.

Similarly, for $J_{N+k}=\left[-\sigma / 3^{k-3},-\sigma / 3^{k-2}\right], k \geq 3$, let $E_{J_{N+k}}=\left[3^{-k}, 3^{-k+1}\right]$ and define $\Lambda_{J_{N+k}}$ as usual as the region in the upper half plane bounded by $J_{N+k}$, $E_{J_{N+k}}$, and the two non-intersecting semicircles joining the endpoints of $J_{N+k}$ to those of $E_{J_{N+k}}$. Then $\left|E_{J_{N+k}}\right|=2 \cdot 3^{N}\left|J_{N+k}\right|$ for $k \geq 3$; the $J_{N+k}$ 's are standard intervals with standard $E_{J_{N+k}}$ 's and $\Lambda_{J_{N+k}}$ 's; the $E_{J_{N+k}}$ 's cover $\left[0, \frac{1}{9}\right]$, and the $\Lambda_{J_{N+k}}$ 's tile the half-disc bounded by $\left[-\sigma, \frac{1}{9}\right]$ and the semicircle in the upper half plane which joins $-\sigma$ to $\frac{1}{9}$. See Figure $5(\mathrm{~b})$.

The Whitney intervals in $(1,1+\sigma]$ are enumerated from right to left in order of decreasing size, as $J_{-(N+3)}, J_{-(N+4)}, \ldots$ Define leaves $\Lambda_{J_{-(N+k)}}$ for $J_{-(N+k)}$, $k \geq 3$, by reflecting the leaves $\Lambda_{J_{N+k}}$ through the line $x=\frac{1}{2}$. The leaf $\Lambda_{J_{-(N+k)}}$ based at $J_{N+k}$ is the mirror image of the leaf $\Lambda_{J_{N+k}}$ based at $J_{N+k}$. All these leaves and their $E_{J_{N+k}}$ 's are standard. The $E_{J_{N+k}}, k \geq 3$, cover $\left[\frac{8}{9}, 1\right]$. 
In the case $L=\overline{\mathbb{R}} \backslash[0,1]$ we have defined standard leaves $\Lambda_{J}$ for all the Whitney intervals $J$ in $L$, except for $J_{\infty}=\overline{\mathbb{R}} \backslash(-\sigma, 1+\sigma)$, which has a non-standard leaf. For all intervals $J \subset L$, the endpoints of $E_{J}$ lie in the Cantor set $K$. The palm leaves have the same properties as those summarized at the end of Case 1.

For each component $L$ of $\overline{\mathbb{R}} \backslash K$, reflect the fan of palm leaves based at $L$ through the real axis, obtaining a fan of palm leaves, also based at $L$, which tiles the lower half plane.

\section{Distortion estimates For Whitney intervals}

Let $\widehat{I}$ be a Whitney interval in the upper orthocircular boundary $\operatorname{arc} A_{n, j}$ of a half fundamental domain $\Omega_{n, j}$. Let $I=P(\widehat{I})$ be the corresponding grid interval, and let $\widetilde{I}$ be the segment of $\partial \Omega_{n, j}$, below $A_{n, j}$, with the same endpoints as $I$. The purpose of this section is to show that the Euclidean lengths of $\widehat{I}, \widetilde{I}$, and $I$ are comparable to each other, with constants which are uniform for all $\widehat{I}$.

We prove a preliminary lemma.

Lemma 10.1. The orthocircular arcs in $\bigcup_{n} \mathcal{A}_{n}$ are uniformly hyperbolically separated.

Proof. It is sufficient to prove that the components of $\overline{\mathbb{R}} \backslash K$ are uniformly hyperbolically separated, since these components lift via $\pi^{-1}$ to $\bigcup_{n} \mathcal{A}_{n}$, and the conformal map $\pi^{-1}$ is a hyperbolic isometry.

Recall that the hyperbolic metric on $\Omega=\overline{\mathbf{C}} \backslash K$, given by $\lambda_{\Omega}(w)|d w|$, satisfies

$$
\frac{c_{\Omega}}{\operatorname{dist}(w, K)} \leq \lambda_{\Omega}(w) \leq \frac{2}{\operatorname{dist}(w, K)}
$$

where $c_{\Omega}>0$ depends only on the uniformly perfect constant of the Cantor set $K$.

Let $L$ and $L^{\prime}$ be components of $\overline{\mathbb{R}} \backslash K$, with $|L| \leq\left|L^{\prime}\right|$. Let $\gamma$ be an arc joining $L$ to $L^{\prime}$. Then the Euclidean length of $\gamma$ is at least $|L|$, since between $L$ and $L^{\prime}$ there is a closed construction interval of $K$ of length at least $|L|$.

If each point in $\gamma$ is within distance $2|L|$ of $K$, then

$$
\begin{aligned}
\ell_{\text {hyp }}(\gamma) & =\int_{\gamma} \lambda_{\Omega}(w)|d w| \\
& \geq c_{\Omega} \int_{\gamma} \frac{|d w|}{\operatorname{dist}(w, K)} \\
& \geq c_{\Omega} \int_{\gamma} \frac{|d w|}{2|L|} \\
& \geq c_{\Omega} / 2 .
\end{aligned}
$$

If some point in $\gamma$ is not within distance $2|L|$ of $K$, then there is a segment $\gamma^{\prime}$ of $\gamma$ of length $|L|$, which has one endpoint at distance $2|L|$ from $K$, and which stays 
within distance $2|L|$ of $K$. Then

$$
\begin{aligned}
\ell_{\mathrm{hyp}}(\gamma) & \geq \ell_{\mathrm{hyp}}\left(\gamma^{\prime}\right) \\
& =\int_{\gamma^{\prime}} \lambda_{\Omega}(w)|d w| \\
& \geq c_{\Omega} \int_{\gamma^{\prime}} \frac{|d w|}{\operatorname{dist}(w, K)} \\
& \geq \frac{c_{\Omega}}{2|L|}|L| \\
& =\frac{c_{\Omega}}{2}
\end{aligned}
$$

Therefore the hyperbolic distance between any two orthocircular arcs in $\bigcup_{n} \mathcal{A}_{n}$ is at least $c_{\Omega} / 2$.

Lemma 10.2. Let $I \in \mathcal{H}$ be any grid interval. Then $|\widehat{I}| \stackrel{c}{\sim}|\widetilde{I}| \stackrel{\frac{\pi}{2}}{\sim}|I|$, and the constant $c>0$ is independent of $I$.

We split the proof into several sublemmas, showing that $|\widehat{I}| \leq c|I|$ for all standard and non-standard grid intervals, and then that $|\widehat{I}| \geq c|I|$ for all standard and non-standard grid intervals. As usual, $c$ denotes constants which may change from line to line.

Note that if $A_{n, j}$ is any orthocircular arc, then its length is comparable with constant $\pi / 2$ to the length of the arc of $\partial \mathbb{D}$ below $A_{n, j}$ which has the same endpoints as $A_{n, j}$. For each $I \in \mathcal{H}$, the endpoints of $\widetilde{I}$ are the endpoints of $I$, and they lie in $\partial \mathbb{D}$. Therefore $\widetilde{I}$ consists of a subset of $\partial \mathbb{D}$ together with a union of whole orthocircular arcs, and so $|\widetilde{I}| \sim|I|$ with constant $\pi / 2$.

Lemma 10.3. $|\widehat{I}| \leq c|I|$ for all standard intervals $I \in \mathcal{H}$.

Proof. Let $A_{n, j}$ be the orthocircular arc containing $\widehat{I}$, and let $\Omega_{n, j}$ be the half fundamental domain below $A_{n, j}$. Without loss of generality, assume that $\pi\left(\Omega_{n, j}\right)$ is the upper half plane. Let $\mathcal{F}$ be the fundamental domain which consists of $\Omega_{n, j}$, the $\operatorname{arc} A_{n, j}$, and the half fundamental domain immediately above $A_{n, j}$. Let $L=$ $\pi\left(A_{n, j}\right)$. Then $\pi(\mathcal{F})$ is the union of the open upper and lower half planes together with the component $L$ of $\overline{\mathbb{R}} \backslash K$; its boundary is $\partial \pi(\mathcal{F})=\overline{\mathbb{R}} \backslash L$.

Let $J=\pi(\widehat{I}) ; J$ is a standard Whitney interval in $\overline{\mathbb{R}} \backslash K$. As usual, let $E_{J}$ be the tip of the standard leaf based at $J ; E_{J}=\pi(\widetilde{I})$. See Figure 6 .

Let $w_{J}$ be the midpoint of $J$ and let $w_{I}$ be the point in $\widehat{I}$ such that $\pi\left(w_{I}\right)=w_{J}$. Let $\alpha \leq|J| / 2$ be a small number, which will be fixed later. Let $z_{J}$ be the point in the upper half plane, directly above the midpoint $w_{J}$ of $J$, such that $\left|z_{J}-w_{J}\right|=\alpha|J|$. Let $z_{I}$ be the point in $\Omega_{n, j}$ such that $\pi\left(z_{I}\right)=z_{J}$.

The hyperbolic distance from $z_{I}$ to $w_{I}$ is bounded away from zero and infinity, uniformly for all $\widehat{I}$. The Euclidean ball of radius $|J| / 2$ centred at $w_{J}$, which contains $z_{J}$ and $J$, lies in the set $\{z \mid \operatorname{dist}(J, K) \leq \operatorname{dist}(z, K) \leq 3 \operatorname{dist}(J, K)\}$, since $|J|=$ 


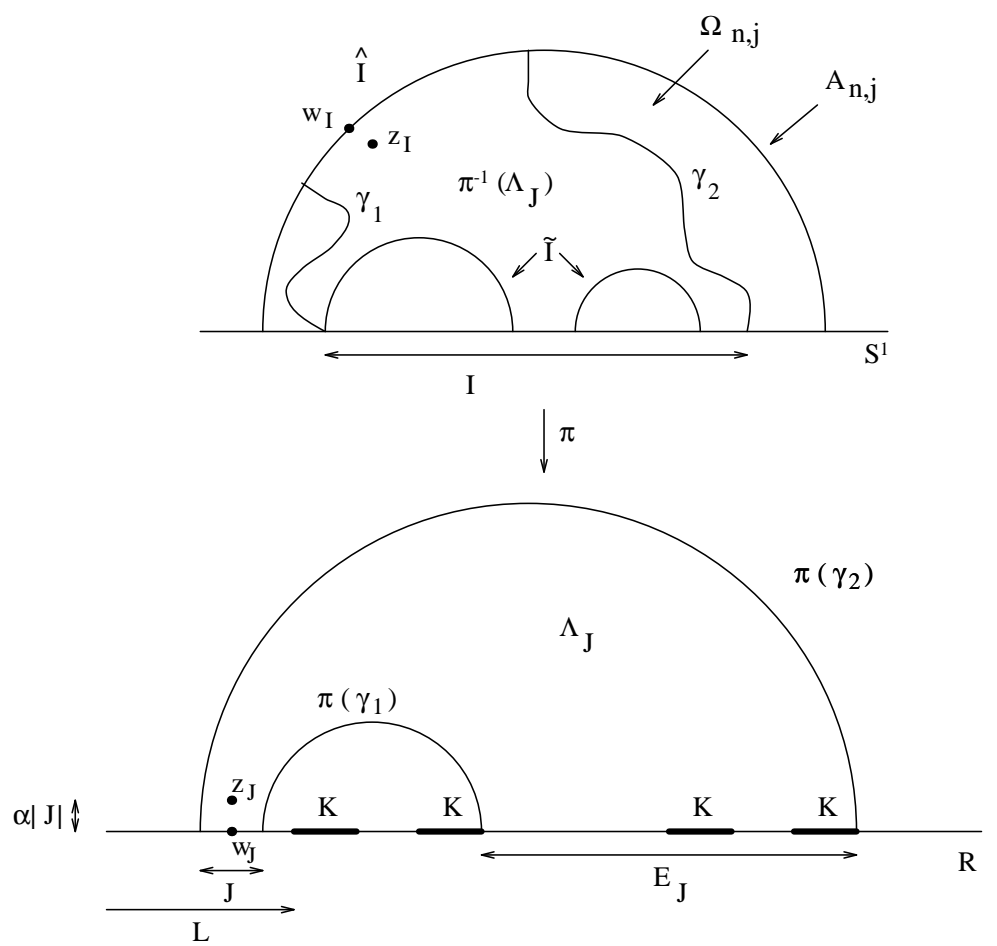

Figure 6. Standard grid interval $I$; definition of $z_{J}$.

$2 \operatorname{dist}(J, K)$. It follows that

$$
\begin{aligned}
d_{\text {hyp }}\left(z_{I}, w_{I}\right) & =d_{\text {hyp }}\left(z_{J}, w_{J}\right) \\
& \leq\left|z_{J}-w_{J}\right| \frac{2}{\operatorname{dist}(J, K)} \\
& =\alpha|J| \frac{2}{|J| / 2} \\
& =4 \alpha .
\end{aligned}
$$

Also,

$$
\begin{aligned}
d_{\text {hyp }}\left(z_{J}, w_{J}\right) & \geq\left|z_{J}-w_{J}\right| \frac{c_{\Omega}}{3 \operatorname{dist}(J, K)} \\
& =\frac{2}{3} c_{\Omega} \alpha .
\end{aligned}
$$

So $\frac{2}{3} c_{\Omega} \alpha \leq d_{\text {hyp }}\left(z_{I}, w_{I}\right) \leq 4 \alpha$ for all $I$. This implies that the Euclidean distance from $z_{I}$ to $w_{I}$ is less than $c(\alpha)\left(1-\left|w_{I}\right|\right)$, where $c(\alpha)$ depends only on $\alpha$ and decreases to zero with $\alpha$.

Since $z_{I}$ and $w_{I}$ are close in the hyperbolic metric, harmonic measure on $\partial \mathcal{F}$ with basepoint $z_{I}$ is close to harmonic measure on $\partial \mathcal{F}$ with basepoint $w_{I}$. Let $u(z)=$ $\omega(z, E, \mathcal{F})$, where $E$ is any Borel subset of $\partial \mathcal{F}$. The function $u(z)$ is harmonic in $\mathcal{F}$. The hyperbolic distance from $w_{I}$ to $\partial \mathcal{F}$ is at least $c_{\Omega} / 2$, by Lemma 10.1. The hyperbolic ball of radius $c_{\Omega} / 2$ centered at $w_{I}$ contains a Euclidean ball of radius $c_{\Omega}^{\prime}\left(1-\left|w_{I}\right|\right)$, centred at $w_{I}$, where $c_{\Omega}^{\prime}$ depends only on $c_{\Omega}$. The Euclidean distance 
from $z_{I}$ to $w_{I}$ decreases to zero with the hyperbolic distance from $z_{I}$ to $w_{I}$. Choose the number $\alpha$, where $\left|z_{I}-w_{I}\right|=\alpha|J|$ and $\left|z_{I}-w_{I}\right| \leq c(\alpha)\left(1-\left|w_{I}\right|\right)$, small enough that the Euclidean distance from $z_{I}$ to $w_{I}$ is less than $\left(c_{\Omega}^{\prime} / 3\right)\left(1-\left|w_{I}\right|\right)$. By Harnack's inequality,

$$
\frac{1}{2} u\left(w_{I}\right) \leq u\left(z_{I}\right) \leq 2 u\left(w_{I}\right) .
$$

In particular, setting $E=\widetilde{I}$, we obtain $\omega\left(z_{I}, \widetilde{I}, \mathcal{F}\right) \stackrel{2}{\sim} \omega\left(w_{I}, \widetilde{I}, \mathcal{F}\right)$ for all $I$.

After these preliminaries, we now use estimates on harmonic measure based at $z_{I}$ and at $w_{I}$ to show that $|\widetilde{I}| \leq c\left(1-\left|w_{I}\right|\right)$. This is sufficient to establish Lemma 10.3, since $|\widehat{I}| \sim 1-\left|w_{I}\right|$ with a constant independent of $I$ (by Lemma 8.1).

For standard intervals $J$, the shapes of the leaves $\Lambda_{J}$ and the position of $z_{J}$ within $\Lambda_{J}$ are all identical, up to dilations and reflections. Specifically, $\left|E_{J}\right|=2 \cdot 3^{N}|J|$; the distance from $J$ to $E_{J}$ is also a fixed multiple of $|J|$; and $z_{J}$ is always at height $\alpha|J|$ above the midpoint of $J$. Therefore the harmonic measure of $E_{J}$ in the upper half plane $\mathbf{U}$, taken with respect to the basepoint $z_{J}$, is constant for all standard $J: \omega\left(z_{J}, E_{J}, \mathbf{U}\right)=c>0$.

Let $\mathcal{F}_{\widetilde{I}}$ be the unit disc without the fundamental domains below $\widetilde{I}$. Let $\gamma$ be the orthocircular arc joining the endpoints of $\widetilde{I}$, and let $\mathcal{F}_{\gamma}$ be the disc without the "bite" below $\gamma$. So $\Omega_{n, j} \subset \mathcal{F} \subset \mathcal{F}_{\gamma} \subset \mathcal{F}_{\widetilde{I}}$. Then

$$
\begin{aligned}
c & =\omega\left(z_{J}, E_{J}, \mathbf{U}\right) \\
& =\omega\left(z_{I}, \widetilde{I}, \Omega_{n, j}\right) \\
& \leq \omega\left(z_{I}, \widetilde{I}, \mathcal{F}\right) \\
& \leq 2 \omega\left(w_{I}, \widetilde{I}, \mathcal{F}\right) \\
& \leq 2 \omega\left(w_{I}, \widetilde{I}, \mathcal{F}_{\widetilde{I}}\right) \\
& =2\left[1-\omega\left(w_{I}, \partial \mathcal{F}_{\widetilde{I}} \backslash \widetilde{I}, \mathcal{F}_{\widetilde{I}}\right)\right] \\
& \leq 2\left[1-\omega\left(w_{I}, \partial \mathcal{F}_{\widetilde{I}} \backslash \widetilde{I}, \mathcal{F}_{\gamma}\right)\right] \\
& =2\left[1-\omega\left(w_{I}, \partial \mathcal{F}_{\gamma} \backslash \gamma, \mathcal{F}_{\gamma}\right)\right] \\
& =2 \omega\left(w_{I}, \gamma, \mathcal{F}_{\gamma}\right) .
\end{aligned}
$$

We have used the conformal invariance of harmonic measure; the observations made above; and the fact that harmonic measure $\omega(z, E, \Omega)$ is monotonic in the domain $\Omega$ : if $z \in \Omega \subset \Omega^{\prime}$ and $E \subset \partial \Omega \cap \partial \Omega^{\prime}$, then $\omega(z, E, \Omega) \leq \omega\left(z, E, \Omega^{\prime}\right)$.

We assumed here that $w_{I}$ was not on or below the orthocircular arc $\gamma$ which joins the endpoints of $I$. Otherwise, $|I| \geq|\gamma| / 2 \geq 1-\left|w_{I}\right|$ and we are done.

Let $\tau: \mathbb{D} \rightarrow \mathbb{D}$ be the Möbius transformation $\tau(z)=\left(z-w_{I}\right) /\left(1-\bar{w}_{I} z\right)$ which takes $w_{I}$ to 0 . See Figure 7 . Then

$$
\begin{aligned}
\omega\left(w_{I}, \gamma, \mathcal{F}_{\gamma}\right) & =\omega\left(0, \tau(\gamma), \tau\left(\mathcal{F}_{\gamma}\right)\right) \\
& =2 \omega(0, \tau(I), \mathbb{D}) \\
& =\pi^{-1}|\tau(I)|
\end{aligned}
$$

The second step can be justified by mapping $\tau(\gamma)$ to a diameter of $\mathbb{D}$ via a Möbius transformation $\sigma: \mathbb{D} \rightarrow \mathbb{D}$ (Figure 7). Consider the probability that a Brownian traveller from $\sigma(0)$ in $\mathbb{D}$ first hits $\partial \mathbb{D}$ somewhere on $\sigma \circ \tau(I)$. By symmetry, this 


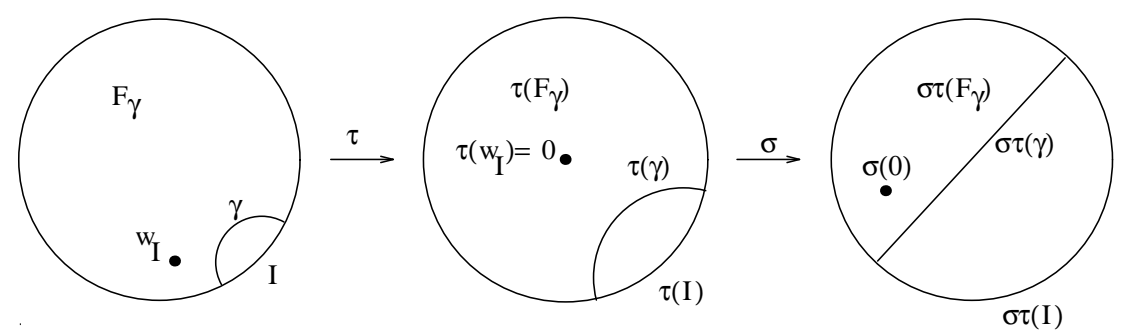

Figure 7. Möbius transformations $\tau, \sigma: \mathbb{D} \rightarrow \mathbb{D}$.

is exactly half the probability that the traveller first hits the boundary of the half $\operatorname{disc} \sigma \circ \tau\left(\mathcal{F}_{\gamma}\right)$ somewhere on the diameter $\sigma \circ \tau(\gamma)$. That is,

$$
\begin{aligned}
\omega(0, \tau(I), \mathbb{D}) & =\omega(\sigma(0), \sigma \circ \tau(I), \mathbb{D}) \\
& =2^{-1} \omega\left(\sigma(0), \sigma \circ \tau(\gamma), \sigma \circ \tau\left(\mathcal{F}_{\gamma}\right)\right) \\
& =2^{-1} \omega\left(0, \tau(\gamma), \tau\left(\mathcal{F}_{\gamma}\right)\right),
\end{aligned}
$$

as required.

We have shown that $c \leq 2 \omega\left(w_{I}, \gamma, \mathcal{F}_{\gamma}\right) \leq 2 \pi^{-1}|\tau(\gamma)|$. Now $\tau^{\prime}(z)=$ $\left(1-\left|w_{I}\right|^{2}\right) /\left(1-w_{I} z\right)^{2}$, and so

$$
\begin{aligned}
|\tau(I)| & \leq|I| \max _{z \in I}\left|\tau^{\prime}(z)\right| \\
& \leq|I| \frac{2}{1-\left|w_{I}\right|} .
\end{aligned}
$$

It follows that $|I| \geq c\left(1-\left|w_{I}\right|\right)$, where the constant $c$ is independent of $I$, and therefore $|I| \geq c|\widehat{I}|$, with $c$ independent of $I$.

Lemma 10.4. $|\widehat{I}| \leq c|I|$ for all non-standard intervals $I \in \mathcal{H}$.

Proof. We use the same proof as for Lemma 10.3 above. The key point is to choose a suitable point $z_{J}$ in the upper half plane $\mathbf{U}$ for each $J$.

Let $J$ be a non-standard Whitney interval. $J$ is of one of two types: either $J$ is the central Whitney interval $J_{c}$ in an open component $L$ of $[0,1] \backslash K$, or $J=J_{\infty}$. In the first case, define $z_{J}$ as in the proof of Lemma 10.3, with the same $\alpha$. See Figure $8(\mathrm{a})$. The leaves $\Lambda_{J}$ for these intervals are identical up to dilations, so the harmonic measure of $E_{J}$ in $\mathbf{U}$, taken from $z_{J}$, is uniformly bounded below for all these $J$. (The bound is not necessarily the same as that in Lemma 10.3.) Also, $z_{J}$ is close enough to $w_{J}$ in the hyperbolic metric that the other estimates on harmonic measure hold with the same constants as in Lemma 10.3 .

For $J=J_{\infty}$, fix a point $w_{J} \in J_{\infty}$ which is far from [0,1]. See Figure 8(b).

Let $z_{J}$ be a point in $\mathbf{U}$, directly above $w_{J}$, which is close to $w_{J}$ in the hyperbolic metric. Then the harmonic measure of $E_{J}$ in $\mathbf{U}$, taken from $z_{J}$, is a positive constant, not necessarily the same as the lower bound in the earlier cases. Choose $z_{J}$ sufficiently close to $w_{J}$ that the other harmonic measure estimates hold with the same constants as in the proof of Lemma 10.3. 


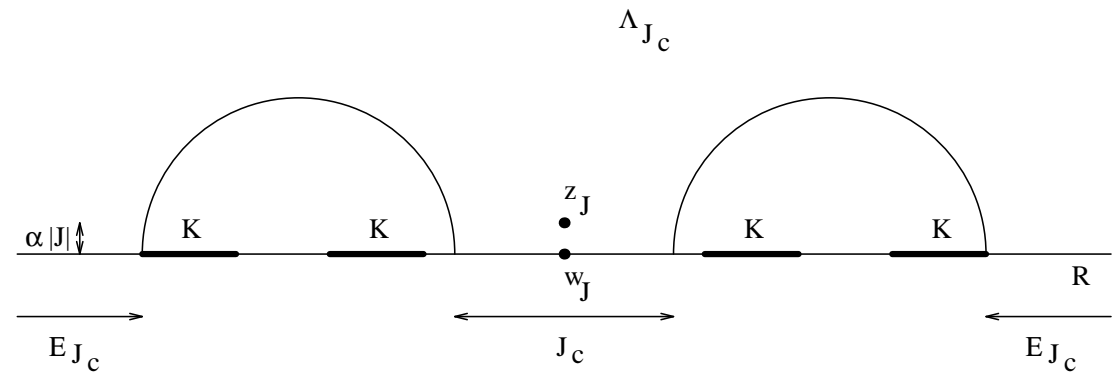

Figure 8(a). Definition of $z_{J}$ for non-standard $J=J_{c}$.

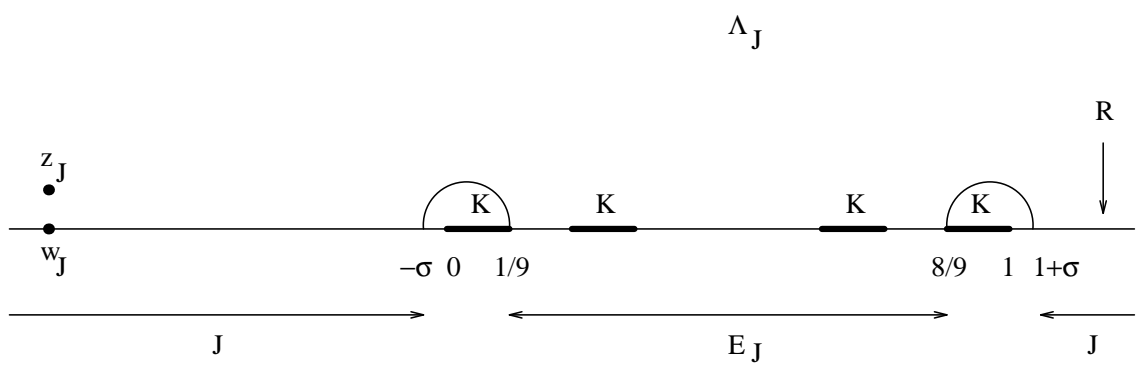

Figure $8(\mathrm{~b})$. Definition of $z_{J}$ for non-standard $J=J_{\infty}$.

Lemma 10.5. $|\widehat{I}| \geq c|I|$ for all standard intervals $I \in \mathcal{H}$.

Proof. We use the same notation as in the proof of Lemma 10.3. In particular, $w_{I}$ is the point in $\widehat{I}$ such that $w_{J}=\pi\left(w_{I}\right)$ is the midpoint of the Whitney interval $J=\pi(\widehat{I})$. Without loss of generality assume that $w_{I}$ is in the left half of the orthocircular arc $A_{n, j}$ containing $\widehat{I}$. Let $a$ and $b$ be the left and right endpoints, respectively, of $I$. See Figure 9.

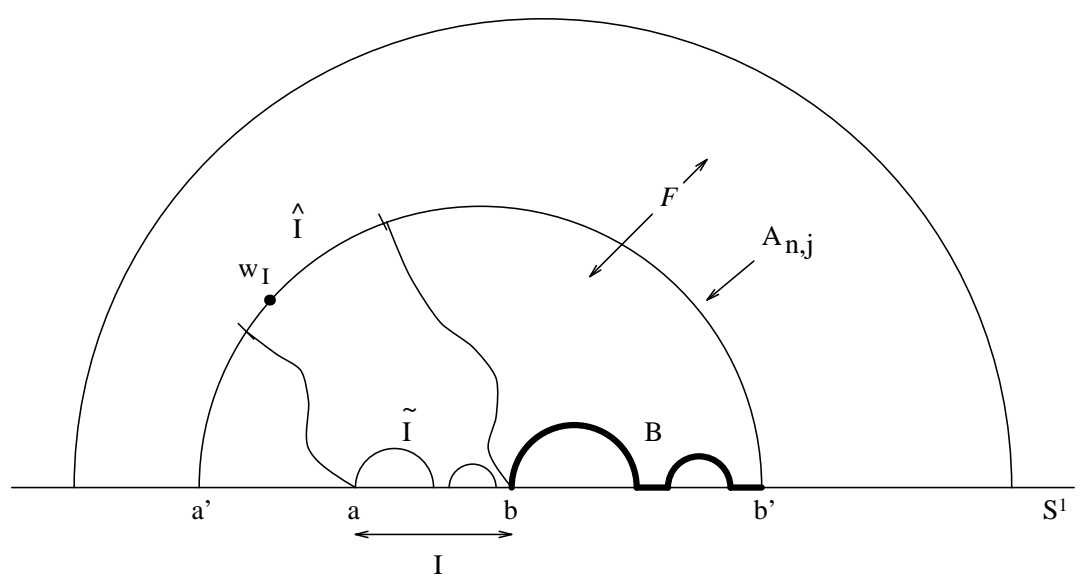

Figure 9. $\widetilde{I}$ is near $\widehat{I}$; definition of $B$. 
It is enough to show that $\left|b-w_{I}\right| \leq c\left(1-\left|w_{I}\right|\right)$ with a constant $c$ independent of $I$. For $I$ is trapped between $b$ and the left endpoint $a^{\prime}$ of $A_{n, j}$, which satisfies $\left|a^{\prime}-w_{I}\right| \leq 2\left(1-\left|w_{I}\right|\right)$. So then

$$
\begin{aligned}
|I| & \leq\left|a^{\prime}-b\right| \\
& \leq\left|a^{\prime}-w_{I}\right|+\left|w_{I}-b\right| \\
& \leq c\left(1-\left|w_{I}\right|\right) \\
& \leq c|\widehat{I}|
\end{aligned}
$$

(using Lemma 8.1), with a constant $c$ independent of $I$.

Let $B$ be the shorter segment of $\partial \mathcal{F}$ between the right endpoint $b$ of $I$ and the right endpoint $b^{\prime}$ of $A_{n, j}$. See Figure 9 . The following calculation is justified below:

$$
\begin{aligned}
\left|w_{I}-b\right| & \leq c \operatorname{dist}\left(w_{I}, B\right) \\
& \leq c \frac{\operatorname{dist}\left(w_{I}, \partial \mathcal{F}\right)}{\omega\left(w_{I}, B, \mathcal{F}\right)^{2}} \\
& \leq c \operatorname{dist}\left(w_{I}, \partial \mathcal{F}\right) \\
& \leq c\left(1-\left|w_{I}\right|\right),
\end{aligned}
$$

where dist denotes Euclidean distance, and $c$ denotes constants independent of $I$.

The Euclidean distance from $w_{I}$ to $B$ is $\left|w_{I}-b\right|$, unless there is a point in an orthocircular arc in $B$ which lies closer to $w_{I}$ than $b$ does. If $\left|b-w_{I}\right| \leq 10\left(1-\left|w_{I}\right|\right)$, the lemma is proved. If not, $B$ is far to the right of $w_{I}$ and the distance from $w_{I}$ to any point in $B$ is at least $\frac{1}{2}\left|w_{I}-b\right|$, justifying the first line of (10.12).

By Beurling's Lemma [A], there is a constant $C>0$ such that for each point $z_{0}$ in $\mathcal{F}$, and for all $M>0$,

$$
\omega\left(z_{0},\left\{z|| z-z_{0} \mid \geq M \operatorname{dist}\left(z_{0}, \partial \mathcal{F}\right)\right\}, \mathcal{F}\right) \leq C M^{-\frac{1}{2}} .
$$

Setting $z_{0}=w_{I}$ and $M=\operatorname{dist}\left(w_{I}, B\right) / \operatorname{dist}\left(w_{I}, \partial \mathcal{F}\right)$, we find

$$
\begin{aligned}
\omega\left(w_{I}, B, \mathcal{F}\right) & \leq \omega\left(w_{I},\left\{z|| z-w_{I} \mid \geq \operatorname{dist}\left(z_{0}, B\right)\right\}, \mathcal{F}\right) \\
& \leq C\left[\frac{\operatorname{dist}\left(w_{I}, \partial \mathcal{F}\right)}{\operatorname{dist}\left(w_{I}, B\right)}\right]^{\frac{1}{2}},
\end{aligned}
$$

and so

$$
\operatorname{dist}\left(w_{I}, B\right) \leq C \frac{\operatorname{dist}\left(w_{I}, \partial \mathcal{F}\right)}{\omega\left(w_{I}, B, \mathcal{F}\right)^{2}},
$$

which is the second line of 10.12 .

The harmonic measure of $B$ in $\mathcal{F}$, measured from $w_{I}$, is bounded below by a positive constant independent of $I$. With $z_{I}$ as in the proof of Lemma 10.3,

$$
\begin{aligned}
\omega\left(w_{I}, B, \mathcal{F}\right) & \geq 2^{-1} \omega\left(z_{I}, B, \mathcal{F}\right) \\
& =2^{-1} \omega\left(z_{J}, \pi(B), \pi(\mathcal{F})\right) \\
& \geq 2^{-1} \omega\left(z_{J}, \pi(B), \mathbf{U}\right),
\end{aligned}
$$

by (10.6) and since $\pi(\mathcal{F})$ contains $\mathbf{U}$. The interval $\pi(B)$ is one of the two components of $\overline{\mathbb{R}} \backslash\left(L \cup E_{J}\right)$, where $L$ is the open component of $\overline{\mathbb{R}} \backslash K$ which contains $J$. Again, since the picture of $\mathbf{U}$ with $J, z_{J}$, and $E_{J}$ is invariant up to dilations and reflections for all standard $J$, the harmonic measure of $\pi(B)$ in $\mathbf{U}$, taken from $z_{J}$, is bounded below by a constant $c>0$ independent of $J$, for all standard $J$. This 
justifies the third line of (10.12). The last line of (10.12) is true because $\widehat{I}$ is a Whitney interval in $A_{n, j}$.

Lemma 10.6. $|\widehat{I}| \geq c|I|$ for all non-standard intervals $I \in \mathcal{H}$.

Proof. We use the same method as for Lemma 10.5. Define $z_{J}$ for each nonstandard $J$ as in the proof of Lemma 10.4. The interval $\pi(B)$ is one of the two components of $\overline{\mathbb{R}} \backslash\left(L \cup E_{J}\right)$. See Figures 8(a) and 8(b). By the usual arguments we may conclude that the harmonic measure of $B$ in $\mathcal{F}$, taken from $w_{I}$, is bounded below by a positive constant independent of $I$. The rest of the proof applies without change.

This completes the proof of Lemma 10.2: $|\widehat{I}| \sim|\widetilde{I}| \sim|I|$ for all grid intervals $I \in \mathcal{H}$, with constants which are independent of $I$.

\section{Definition of the Density functions $F_{n} \cdots F_{1}$}

In this section we define the functions $F_{n}$ whose products $F_{n} \cdots F_{1}$ give the densities of the measures $\mu_{n}$. Here we define the $F_{n}$ on all standard grid intervals $I \in \mathcal{H}_{n-1}, n \geq 1$, up to the values of certain parameters, $Q$ and $\varepsilon$, which will be fixed later. We show that these functions $F_{n}$ are $(\delta, \eta)$-suitable for each standard $I \in \mathcal{H}_{n-1}$, with constants $\delta$ and $\eta$ independent of $I$ and $n$. For non-standard intervals $I \in \mathcal{H}_{n-1}$, we define the functions $F_{n}$ and prove that they are $(\delta, \eta)$ suitable in Section 14.

We first prove a lemma giving a rough estimate of the distortion caused by the composition $P \circ \pi^{-1}$ of a branch of the inverse $\pi^{-1}$ of the covering map with the almost radial projection $P$ of the disc onto the circle.

Lemma 11.1. For each standard Whitney interval $J$ in $\overline{\mathbb{R}} \backslash K$, let $S_{J}$ be a segment of $E_{J}$, and let $A_{1}$ and $A_{2}$ be the components (possibly empty) of $E_{J} \backslash S_{J}$. Suppose that $\left|S_{J}\right| /\left|E_{J}\right|=c_{1},\left|A_{1}\right| /\left|E_{J}\right|=c_{2}$, and $\left|A_{2}\right| /\left|E_{J}\right|=1-c_{1}-c_{2}$, where $c_{1}$ and $c_{2}$ are constants independent of $J$. Suppose also that $S_{J}$ is a union of whole Whitney intervals, possibly together with a subset of $K$. Let $I$ be any standard grid interval such that $\pi(\widehat{I})=J$, and let $\widehat{R_{I}}$ be the segment of $\widehat{I}$ such that $\pi\left(\widehat{R_{I}}\right)=S_{J}$. Let $R_{I}=P\left(\widehat{R_{I}}\right)=P \circ \pi^{-1}\left(S_{J}\right)$. Then $\left|R_{I}\right| \geq c|I|$, where $c$ is a constant independent of $I$ and $J$.

Proof. Without loss of generality, assume that $\pi$ maps the half fundamental domain below the orthocircular arc containing $\widehat{I}$ to the upper half plane. As in the proof of Lemma 10.3, let $w_{J}$ be the midpoint of $J$, and let $z_{J}$ be the point in the upper half plane, directly above $w_{J}$, such that $\left|z_{J}-w_{J}\right|=\alpha|J|$, where $\alpha$ is a small fixed number independent of $J$. Let $w_{I}$ be the point in $\widehat{I}$ such that $\pi\left(w_{I}\right)=w_{J}$. See Figure 6 in Section 10.

The harmonic measure of $S_{J}$ in the upper half plane, taken from $z_{J}$, is the same for all $J$, since $S_{J}$ is always in the same place in $E_{J}$. The proof of Lemma 10.3, applied to $S_{J}$ instead of to $E_{J}$, shows that $\left|R_{I}\right| \geq c\left(1-\left|w_{I}\right|\right)$, where $c$ is a constant depending on $c_{1}$ and $c_{2}$. By Lemmas 8.1 and 10.2, $1-\left|w_{I}\right|$ is comparable to $|I|$ with a constant independent of $I$ and $J$, which proves the lemma.

We have shown that for all standard intervals $I \in \mathcal{H}$, if $S_{J}$ is a fixed segment of $E_{J}$, where $J=\pi(\widehat{I})$, then $\left|R_{I}\right| /|I|=\left|P \circ \pi^{-1}\left(S_{J}\right)\right| /\left|P \circ \pi^{-1}\left(E_{J}\right)\right|$ is uniformly bounded away from zero. The same result holds for non-standard intervals $I$, if $S_{J}$ 


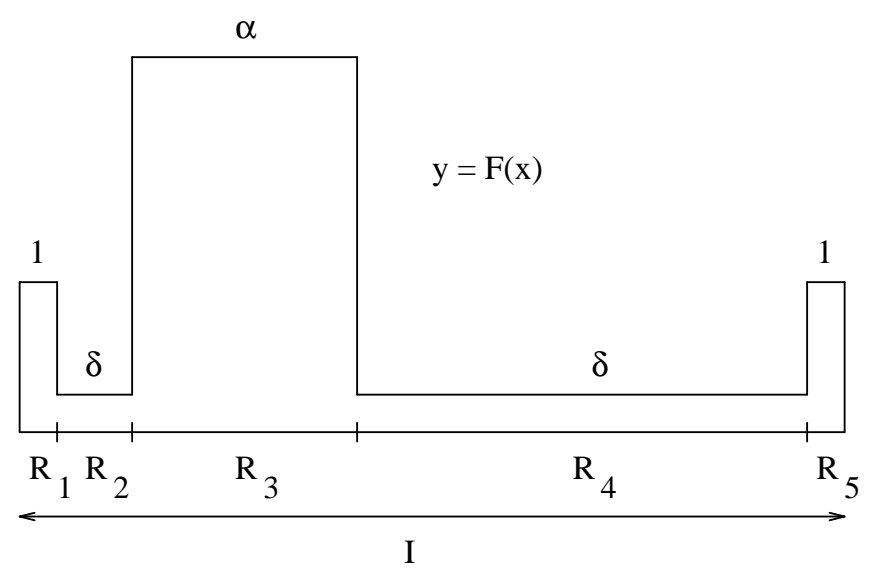

FiguRE 10. Graph of $F ; \alpha=(1-\varepsilon)|I| /\left|R_{3}\right|$.

is defined so that its length is a fixed multiple of $|J|$ and so that it is always in the same position in $E_{J}$.

On each interval $I$ in the grid $\mathcal{H}$ of subintervals of the unit circle we define a function $F$ which is $(\delta, \eta)$-suitable for $I$, where the constants $\delta$ and $\eta$ are independent of $I$. Recall from Section 6 the definition of $(\delta, \eta)$-suitable: $F$ has mean value one on $I ; 0<\delta \leq F(x) \leq 1 / \delta$ on $I$; and $F \equiv 1$ on subintervals $I_{l}$ and $I_{r}$ of $I$, at the left and right ends respectively of $I$, such that $\left|I_{l}\right| \geq \eta|I|$ and $\left|I_{r}\right| \geq \eta|I|$.

The idea is as follows. Divide each $E_{J}$ into five segments $S_{1}, \ldots, S_{5}$ whose lengths are prescribed fractions of $\left|E_{J}\right|$. Pull these back to $\widetilde{I}$ via $\pi^{-1}$, then project them via $P$ to subintervals $R_{j}=P \circ \pi^{-1}\left(S_{j}\right), 1 \leq j \leq 5$, of $I$. In Lemma 11.1 we gave bounds on the distortion in length caused by $P \circ \pi^{-1}$. Each ratio $\left|R_{j}\right| /|I|$ is uniformly bounded away from zero and infinity for all $I$. Define $F$ to be constant on each $R_{j}$, in such a way that $F$ is large on $R_{3}$, identically equal to one on $R_{1}$ and $R_{5}$, and small enough on $R_{2} \cup R_{4}$ to ensure that $F$ has mean value one on $I$. See Figure 10. We show that $F$ is uniformly bounded away from zero and infinity for all $I$.

Now we define the segments $S_{j}$ and $R_{j}$ for a standard grid interval $I$. Let $\widehat{I}$ be the Whitney interval in $\bigcup_{n} \mathcal{A}_{n}$ such that $I=P(\widehat{I})$, and let $J=\pi(\widehat{I})$. Without loss of generality we describe the case when $E_{J}$ is to the right of $J$. Divide $E_{J}$ into five segments $S_{j}, 1 \leq j \leq 5$, numbered from left to right, as follows. The left half of $E_{J}$ is an open component of $\overline{\mathbb{R}} \backslash K$ of length $3^{N}|J|$. Let $S_{3}$ be the central Whitney interval $J_{c}$ in this open interval. The length of $S_{3}$ is $\left|S_{3}\right|=\frac{1}{2}\left(1-3^{-N}\right)\left|E_{J}\right|$.

Let $Q$ be a large integer; its value will be fixed below. Let $S_{1}$ be the interval with the same left endpoint as $E_{J}$ and with length $\left|S_{1}\right|=\frac{3}{2} 3^{-Q}|J| . S_{1}$ is a union of whole Whitney intervals. Let $S_{5}$ be the interval with the same right endpoint as $E_{J}$ and with length $\left|S_{5}\right|=3^{-Q}|J| . \quad S_{5}$ is a closed construction interval of the Cantor set $K$. Let $S_{2}$ be the interval between $S_{1}$ and $S_{3}$, and let $S_{4}$ be the interval between $S_{3}$ and $S_{5}$.

Now pull back these intervals $S_{j}$ to $\widetilde{I}$ via the appropriate branch of $\pi^{-1}$. Then project them to $I$ via $P$, obtaining five intervals $R_{j}=P \circ \pi^{-1}\left(S_{j}\right), 1 \leq j \leq 5$, whose union is $I$. By Lemma 11.1, there are positive constants $c_{j}, 1 \leq j \leq 5$, such 
that $\left|R_{j}\right| \geq c_{j}|I|$ for all standard intervals $I$. These constants are less than one; they depend on $N$ and $Q$ but are independent of $J$ and $I$.

Let $\varepsilon$ be a positive number, small enough that $(1-\varepsilon)|I| /\left|R_{3}\right|>1$; its exact value will be fixed below. Define a step function $F$ on $I$ by

$$
F(x)= \begin{cases}1, & x \in R_{1} \cup R_{5} ; \\ (1-\varepsilon) \frac{|I|}{\left|R_{3}\right|}, & x \in R_{3} ; \\ \delta, & x \in R_{2} \cup R_{4} ;\end{cases}
$$

where $\delta=\left(\varepsilon|I|-\left|R_{1} \cup R_{5}\right|\right) /\left|R_{2} \cup R_{4}\right|$ is chosen so that $\frac{1}{|I|} \int_{I} F=1$. See Figure 10 .

This function $F$ takes its maximum on $R_{3}$, where $F(x) \equiv(1-\varepsilon)|I| /\left|R_{3}\right| \leq 1 / c_{3}$; this upper bound is independent of $I$. The subintervals $R_{1}$ and $R_{5}$ at each end of $I$, on which $F \equiv 1$, each have length at least $\eta|I|$, where $\eta=\min \left(c_{1}, c_{5}\right)$ is independent of $I$.

If $d \mu_{n}=F_{n} \cdots F_{1} d x$, and each $F_{m}$ is defined on each $I \in \mathcal{H}_{m-1}, m \geq 1$, according to (11.1), then most of the mass $\mu_{n-1}(I)$ is concentrated onto the subinterval $R_{3}$ of $I$ :

$$
\begin{aligned}
\mu_{n}\left(R_{3}\right) & =\int_{R_{3}} F_{n}(x) d \mu_{n-1}(x) \\
& =(1-\varepsilon) \frac{|I|}{\left|R_{3}\right|} \mu_{n-1}\left(R_{3}\right) \\
& =(1-\varepsilon) \frac{|I|}{\left|R_{3}\right|} \cdot \frac{\left|R_{3}\right|}{|I|} \mu_{n-1}(I) \\
& =(1-\varepsilon) \mu_{n-1}(I) .
\end{aligned}
$$

The third step is valid because $\mu_{n-1}$ is a constant multiple of Lebesgue measure on $I$.

To show that the function $F$ defined in (11.1) is $\left(\delta_{0}, \eta\right)$-suitable for $I$, with constants $\delta_{0}$ and $\eta$ independent of $I$, it only remains to give a uniform lower bound for the value $\delta$ of $F$ on $R_{2} \cup R_{4}$. Observe that

$$
\delta=\frac{\varepsilon|I|-\left|R_{1} \cup R_{5}\right|}{\left|R_{2} \cup R_{4}\right|}=\left(\varepsilon-\frac{\left|R_{1} \cup R_{5}\right|}{|I|}\right) \frac{|I|}{\left|R_{2} \cup R_{4}\right|} \geq \varepsilon-\frac{\left|R_{1} \cup R_{5}\right|}{|I|} .
$$

We would like to ensure that $\left|R_{1} \cup R_{5}\right| /|I| \leq \varepsilon / 2$, say, by choosing $\left|S_{1} \cup S_{5}\right| /\left|E_{J}\right|$ sufficiently small (that is, by choosing $Q$ sufficiently large). Unfortunately, an application of Lemma 11.1 to $S_{J}=E_{J} \backslash\left(S_{1} \cup S_{5}\right)$ does not guarantee that this can be done, since the lemma does not give a good estimate on the size of the constant $c$, nor on its behaviour as $c_{1} \rightarrow 0$. However, the sharper estimate in Lemma 13.1 below, based on the $A_{\infty}$-equivalence of harmonic measure and arclength on the boundary of a chord-arc domain, does imply that $\left|R_{1} \cup R_{5}\right| /|I|$ goes to zero with $\left|S_{1} \cup S_{5}\right| /\left|E_{J}\right|$. Therefore, we may choose a large $Q$ so that $\left|S_{1} \cup S_{5}\right| /\left|E_{J}\right|=\frac{5}{2} \cdot \frac{1}{2} \cdot 3^{-Q-N}$ is sufficiently small that $\left|R_{1} \cup R_{5}\right| /|I| \leq \varepsilon / 2$. Then $\delta \geq \varepsilon / 2$.

We have shown that the function $F$ defined in $(11.1)$ is $\left(\delta_{0}, \eta\right)$-suitable for $I$, where the constants $\delta_{0}$ and $\eta$ depend on $\varepsilon, N$, and $Q$, but are independent of $I$, for all standard grid intervals $I \in \mathcal{H}$.

In Section 14 we define the function $F$ for non-standard intervals $I \in \mathcal{H}$, and show that it is $\left(\delta_{0}, \eta\right)$-suitable for those $I$. 


\section{Definition of AUXiliary FUnCtions $X_{i}$}

Let $I_{0}$ be an interval from the $l^{\text {th }}$ layer $\mathcal{H}_{l}$ of the grid, such that $\pi\left(\widehat{I_{0}}\right)$ is not $J_{\infty}$. For each point $x \in I_{0}$ and for each $i \geq 0$, let $I_{i}(x)$ be the unique interval in $\mathcal{H}_{l+i}$ which contains $x$. The intervals $I_{i}(x)$ are nested: $I_{0}(x) \supset I_{1}(x) \supset I_{2}(x) \supset \cdots \supset$ $I_{i}(x) \supset \cdots \ni x$. Let $\widehat{I}_{i}(x)$ be the Whitney interval in the disc such that $P\left(\widehat{I}_{i}(x)\right)=$ $I_{i}(x)$. Let $J_{i}(x)=\pi\left(\widehat{I}_{i}(x)\right)$. We have associated to the point $x \in I_{0} \subset \partial \mathbb{D}$ a sequence $\left\{J_{i}(x)\right\}_{i>0}$ of Whitney intervals in $\overline{\mathbb{R}} \backslash K$.

We now define auxiliary functions $X_{i}(x)$ which keep track of the lengths $\left|J_{i}(x)\right|$ of these intervals. Make the convention that

$$
\left|J_{c}\right|=|L| / 3
$$

when $J_{c}$ is the non-standard central Whitney interval in a component $L$ of $[0,1] \backslash K$. Let

$$
X_{1}(x)=\log _{3}\left[\frac{\left|J_{1}(x)\right|}{\left|J_{0}\right|}\right]
$$

for $x \in I_{0}$. For $i \geq 2$, let

$$
X_{i}(x)= \begin{cases}\log _{3}\left[\frac{\left|J_{i}(x)\right|}{\left|J_{i-1}(x)\right|}\right], & \text { if } J_{1}(x), \ldots, J_{i-1}(x) \neq J_{\infty} \\ 1, & \text { otherwise }\end{cases}
$$

for $x \in I_{0}$ and $i \geq 1$. Let

$$
S_{k}(x)=\sum_{i=1}^{k} X_{i}(x)
$$

for $k \geq 1$. If none of $J_{1}(x), \ldots, J_{k-1}(x)$ is the large interval $J_{\infty}=\overline{\mathbb{R}} \backslash(-\sigma, 1+\sigma)$, then $S_{k}(x)=\log _{3}\left(\left|J_{k}(x)\right| /\left|J_{0}\right|\right)$. Note that $X_{i}$ and $S_{k}$ are integer-valued.

Let $V\left(I_{0}\right)$ denote the collection of those grid intervals $I$ in $I_{0}$ which satisfy $\pi(\widehat{I})=J_{\infty}$, and which are maximal with respect to this property. In other words, $V\left(I_{0}\right)$ is the collection of the maximal grid intervals $I$ in $I_{0}$ which are images of $J_{\infty}$ under branches of $P \circ \pi^{-1}$.

Observe that $V\left(I_{0}\right)=\left\{x \in I_{0} \mid S_{k}(x) \rightarrow+\infty\right\}$. For if $x$ is contained in some interval $I=I_{N}(x)$, say, which is in $V\left(I_{0}\right)$, then $X_{i}(x)=1$ for all $i \geq N$, and so $S_{k}(x)$ tends to infinity. And if $x$ is not contained in any interval $I$ in $V\left(I_{0}\right)$, then no $J_{i}(x)$ is $J_{\infty}$, and so $S_{k}(x)$ remains bounded above, by $\log _{3}\left[\left(9\left|J_{0}\right|\right)^{-1}\right]$, for all $k$. (Recall that $J_{0}=\pi\left(\widehat{I_{0}}\right)$ is fixed in this discussion, and that the largest Whitney interval other than $J_{\infty}$ is the $J_{c}$ in $\left(\frac{1}{3}, \frac{2}{3}\right)$, which has length $1 / 9$ by our convention.)

In the remainder of the paper we show that for appropriate choices of the parameters in the definitions of the functions $F_{n}$, the measures $\mu_{n}$ given by $d \mu_{n}=$ $F_{n} \cdots F_{1} d x$ converge to a measure $\mu$ whose restriction to $I_{0}$ is supported on $V\left(I_{0}\right)$. In Sections 13 and 14 we show that for all grid intervals $I \subset I_{0}$, the mean of $X_{i}$ on $I$ (where $i$ is such that $J_{i-1}=\pi(\widehat{I})$ ) with respect to $\mu$ is uniformly large, and the second moment is uniformly small. In Section 15 we conclude that $S_{k} \rightarrow+\infty$ a.e. $(d \mu)$ on $I_{0}$; that is, $\mu\left(V\left(I_{0}\right)\right)=\mu\left(I_{0}\right)$.

Finally, also in Section 15, we observe that this implies the existence of a doubling measure $\mu$ supported on the set $S$ of points which lie in infinitely many grid intervals corresponding to $J_{\infty}$. Heuristically, the grid intervals corresponding to $J_{\infty}$ are near preimages $\widehat{I_{\infty}}$ of $J_{\infty}$ under $\pi$. Each preimage $\widehat{I_{\infty}}$ contains an orbit point $g(0)$, $g \in G$, since the covering map $\pi$ is normalized so that the orbit of 0 is $\pi^{-1}(\infty)$, 
and $\infty \in J_{\infty}$. We prove that $S$ is contained in the conical limit set of $G$, which completes the proof of Theorem 1.2.

13. Estimates $E X_{i} \geq c_{1}$ AND $E X_{i}^{2} \leq c_{2}$ FOR STANDARD InTERVAls

The main result of this section (Lemma 13.4) is that on each standard grid interval $I \in \mathcal{H}$, the function $X_{i}$ satisfies

$$
\mu(I)^{-1} \int_{I} X_{i} d \mu \geq c_{1} \quad \text { and } \quad \mu(I)^{-1} \int_{I} X_{i}^{2} d \mu \leq c_{2},
$$

where $c_{1}$ and $c_{2}$ are positive constants independent of $I$. Here

$$
X_{i}=\log _{3}\left(\left|J_{i}(x)\right| /\left|J_{i-1}(x)\right|\right)
$$

is the auxiliary function, defined in Section 12, for a particle which has not yet reached $J_{\infty}$ and which makes its $i^{\text {th }}$ jump from $J=\pi(\widehat{I})$. We begin by establishing an estimate, sharper than that in Section 11, on the distortion in length caused by the map $P \circ \pi^{-1}$.

As usual, for a standard grid interval $I$ with $I=P(\widehat{I})$, let $J=\pi(\widehat{I})$, and let $E_{J}=\pi(\widetilde{I})$ be the tip of the leaf $\Lambda_{J}$ based at $J$. See Figure 11.
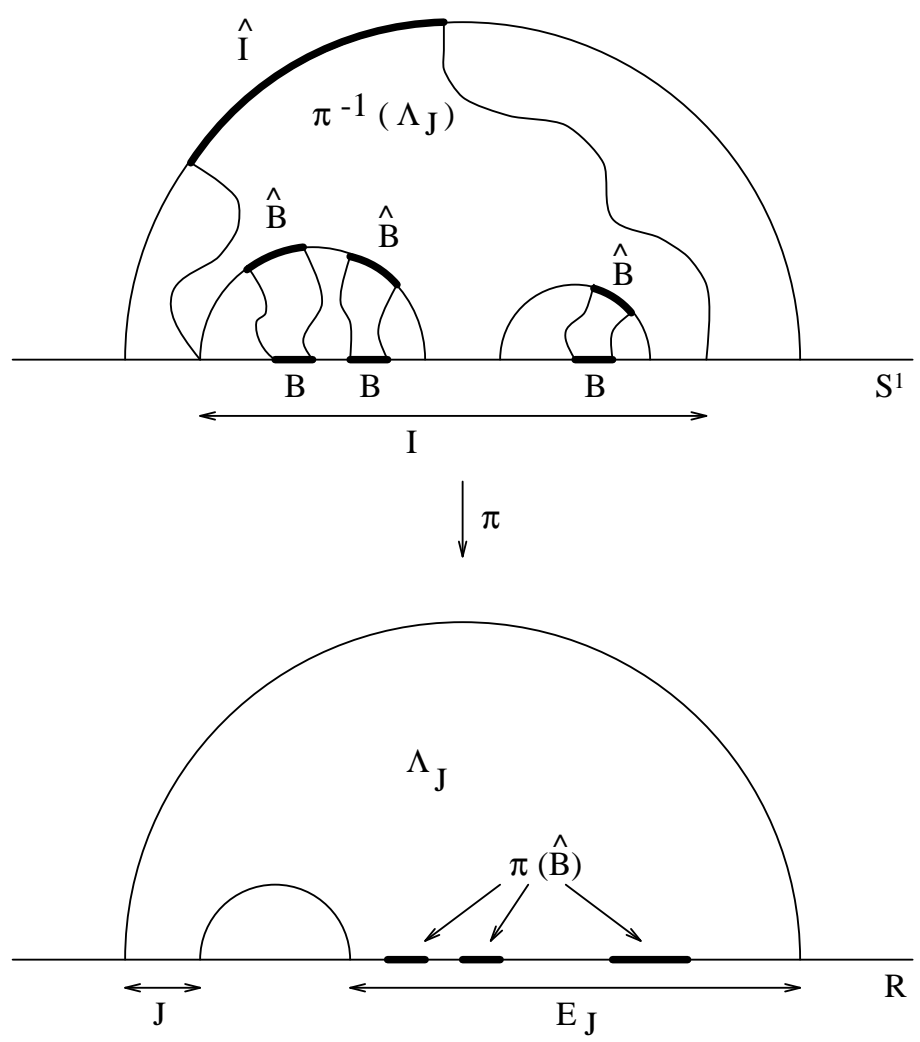

Figure 11. $B$ is in $I ; \pi(\widehat{B})$ is in $E_{J}$. 
Lemma 13.1. Let $I \in \mathcal{H}_{n-1}, n \geq 1$, be a standard grid interval. Let $B \subset I$ be a union of grid intervals from $\mathcal{H}_{n}$. Let $\widehat{B}$ be the subset of $\widetilde{I}$ such that $P(\widehat{B})=B$. There are positive constants $c$ and $\alpha$ such that

$$
\frac{|B|}{|I|} \leq c\left[\frac{|\pi(\widehat{B})|}{\left|E_{J}\right|}\right]^{\alpha} .
$$

The constants $c$ and $\alpha$ are independent of $B, I$, and $n$, but they depend on the large number $N$ such that $\left|E_{J}\right|=2 \cdot 3^{N}|J|$ for standard intervals.

To prove this lemma, we use Lemmas 13.2 and 13.3 below and the following remarks.

A Jordan curve $\Gamma$ is said to be chord-arc if for all points $x$ and $y$ on $\Gamma$ the Euclidean length $|x-y|$ of the chord between $x$ and $y$ is comparable, with a uniform constant, to the Euclidean arclength of the shorter arc of $\Gamma$ between $x$ and $y$. In other words, there is a constant $c>0$ such that

$$
|x-y| \leq \ell_{\Gamma}(x, y) \leq c|x-y|
$$

for all $x, y$ on $\Gamma$. A domain $D$ is a chord-arc domain if its boundary is a chord-arc curve.

The leaves $\Lambda_{J}$ are chord-arc domains with chord-arc constant independent of $J$.

If $D$ is a bounded chord-arc domain, then arclength on its boundary $\partial D$ and harmonic measure based at any point $z$ in $D$ are $A_{\infty}$-equivalent. This means that there are positive constants $c_{1}, c_{2}, \alpha_{1}$, and $\alpha_{2}$ such that whenever $S$ is a segment of $\partial D$ and $E$ is a Borel subset of $S$, then

$$
\frac{|E|}{|S|} \leq c_{1}\left[\frac{\omega(z, E, D)}{\omega(z, S, D)}\right]^{\alpha_{1}} \quad \text { and } \quad \frac{\omega(z, E, D)}{\omega(z, S, D)} \leq c_{2}\left[\frac{|E|}{|S|}\right]^{\alpha_{2}} \text {. }
$$

See [JK]. Moreover, if $D$ is bounded, then for all points $z \in D$ which satisfy

$$
\operatorname{dist}(z, \partial D) \geq C \operatorname{diam}(D),
$$

the constants $c_{1}, c_{2}, \alpha_{1}$, and $\alpha_{2}$ depend only on $C$ and the chord-arc constant of $\partial D$.

In the situation of Lemma 13.1, consider the domain $D=\pi^{-1}\left(\Lambda_{J}\right)$ whose boundary consists of $\widehat{I}, \widetilde{I}$, and two $\operatorname{arcs} \gamma_{1}$ and $\gamma_{2}$ which project via $\pi$ to the two semicircles in the boundary of the leaf $\Lambda_{J}$. We prove (Lemma 13.2) that $\partial D$ is chord-arc, with a constant independent of $I$, and (Lemma 13.3) that the inequality 13.4 holds, with a constant independent of $I$, for the point $z_{I} \in D$ defined in Section 10 . Then we prove Lemma 13.1.

Lemma 13.2. The boundary $\partial D$ of $D$ is chord-arc, with a chord-arc constant which is independent of $I$.

Proof. We follow the proof of a similar result by González $[\mathrm{G}]$. The boundary of $D$ consists of $\widehat{I}, \gamma_{1}, \gamma_{2}$, the orthocircular $\operatorname{arcs}$ in $\widetilde{I}$, and the subset $\widetilde{I} \cap \partial \mathbb{D}$ of the unit circle. It is sufficient to show that:

1. Wherever two of $\widehat{I}, \gamma_{1}, \gamma_{2}$, and $\widetilde{I}$ meet, the angle they form is bounded below by some $\theta_{0}>0$ which is independent of $I$; and

2. The components of $\partial \Lambda_{J} \backslash K$ (which are $J=\pi(\widehat{I})$; the semicircles $\pi\left(\gamma_{1}\right)$ and $\pi\left(\gamma_{2}\right)$; and the open intervals in $\left.E_{J} \backslash K\right)$ are chord-arc, in the hyperbolic metric, with a chord-arc constant which is independent of $I$. 

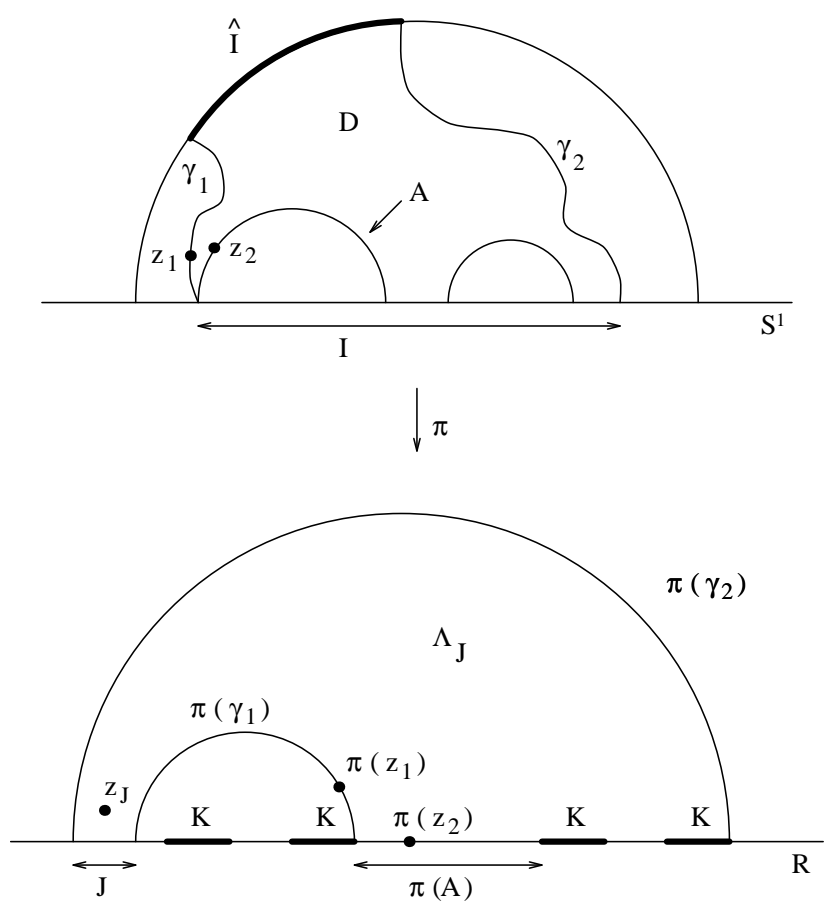

Figure 12. $\Lambda_{J}$ and $D=\pi^{-1}\left(\Lambda_{J}\right)$ are chord-arc domains.

Then the components of $\partial D \backslash \partial \mathbb{D}$ are also chord-arc in the hyperbolic metric; they are chord-arc in the Euclidean metric; and the whole boundary $\partial D$ is chord-arc in the Euclidean metric with a constant depending only on the chord-arc constant of $\Lambda_{J}$.

1. $\widehat{I}$ meets $\gamma_{1}$ and $\gamma_{2}$ at right angles, because $J$ meets $\pi\left(\gamma_{1}\right)$ and $\pi\left(\gamma_{2}\right)$ at right angles and $\pi^{-1}$ is conformal.

Let $\gamma_{1}$ be the arc in $\partial D$ such that $\pi\left(\gamma_{1}\right)$ is the smaller semicircle in $\partial \Lambda_{J}$, and let $A$ be the orthocircular arc in the lower part of $\partial D$ which meets $\gamma_{1}$. See Figure 12 . We show that the hyperbolic distance between any two points $z_{1} \in \gamma_{1}$ and $z_{2} \in A$ is uniformly bounded away from zero; this implies that $\gamma_{1}$ and $A$ do not form a cusp at their common endpoint but make a positive angle there. Take a path from $\pi\left(z_{1}\right)$ to $\pi\left(z_{2}\right)$ in $\Lambda_{J}$. Let $\gamma$ be the segment of this path which starts at $\pi\left(z_{1}\right)$ and has length $\operatorname{Im}\left(z_{1}\right) / 2$. Then

$$
\begin{aligned}
d_{\text {hyp }}\left(z_{1}, z_{2}\right) & =d_{\text {hyp }}\left(\pi\left(z_{1}\right), \pi\left(z_{2}\right)\right) \\
& \geq c_{\Omega} \int_{\gamma} \frac{|d w|}{\operatorname{dist}(w, K)} \\
& \geq c_{\Omega} \frac{1}{\frac{3}{2} \operatorname{Im} \pi\left(z_{1}\right)} \cdot \frac{\operatorname{Im} \pi\left(z_{1}\right)}{2} \\
& \geq c_{\Omega} / 3
\end{aligned}
$$

So the angle formed by $\gamma_{1}$ and $A$ is bounded below by some $\theta_{0}>0$, independent of $I$. 
The same reasoning applied to $\gamma_{2}$ shows that $\gamma_{2}$ makes a positive angle, uniformly bounded below, with $\partial \mathbb{D}$.

2. $J$ and the open intervals in $E_{J} \backslash K$ are geodesic arcs in $\Omega=\overline{\mathbf{C}} \backslash K$, so they are chord-arc in the hyperbolic metric. To show that the semicircle $\pi\left(\gamma_{1}\right)$ is hyperbolically chord-arc, take points $z$ and $\zeta$ in $\pi\left(\gamma_{1}\right)$. Let $\gamma$ be the segment of $\pi\left(\gamma_{1}\right)$ between $z$ and $\zeta$, and let $L$ be the chord between $z$ and $\zeta$. It is enough to consider $z$ and $\zeta$ in the part of $\pi\left(\gamma_{1}\right)$ near (in the Euclidean metric) to an endpoint of $\pi\left(\gamma_{1}\right)$. Then $\gamma$ is almost vertical, and

$$
\ell_{\mathrm{hyp}}(\gamma) \sim \int_{\gamma} \frac{|d w|}{\operatorname{dist}(w, K)} \sim \int_{\gamma} \frac{|d w|}{\operatorname{Im} w}
$$

is comparable to

$$
\ell_{\text {hyp }}(L) \sim \int_{L} \frac{|d w|}{\operatorname{dist}(w, K)} \sim \int_{L} \frac{|d w|}{\operatorname{Im} w}
$$

with a uniform constant.

This completes the proof of Lemma 13.2.

Lemma 13.3. For each standard grid interval $I \in \mathcal{H}$, let $z_{I}$ be the point in $D$, near $\widehat{I}$, defined in Section 10. There is a constant $C>0$ independent of I such that

$$
\operatorname{dist}\left(z_{I}, \partial D\right) \geq C \operatorname{diam}(D) \text {. }
$$

Proof. We compare both sides of (13.8) to $1-\left|w_{I}\right|$, where $w_{I}$ is the point in $\widehat{I} \subset \partial D$, near $z_{I}$, defined in Section 10. (See Figure 6.)

The hyperbolic distance from $z_{I}$ to $\partial D$ is uniformly bounded away from zero. To see this, consider a geodesic arc from $z_{J}=\pi\left(z_{I}\right)$ to $\partial \Lambda_{J}$. Let $\gamma$ be the segment of this geodesic which has $z_{J}$ as one endpoint and which has Euclidean length $\alpha|J| / 2$. Then

$$
\begin{aligned}
d_{\mathrm{hyp}}\left(z_{I}, \partial D\right) & =d_{\mathrm{hyp}}\left(\pi\left(z_{I}\right), \pi(\partial D)\right) \\
& =d_{\mathrm{hyp}}\left(z_{J}, \partial \Lambda_{J}\right) \\
& \geq c_{\Omega} \int_{\gamma} \frac{|d z|}{\operatorname{dist}(z, K)} \\
& \geq c_{\Omega} \cdot \frac{1}{\frac{3}{2} \alpha|J|+\frac{1}{2}|J|} \cdot \frac{\alpha|J|}{2} \\
& =c(\Omega, \alpha) .
\end{aligned}
$$

The hyperbolic ball of radius $c(\Omega, \alpha)$ centred at $z_{I}$ contains a Euclidean ball of radius $c^{\prime}\left(1-\left|z_{I}\right|\right)$, centred at $z_{I}$. Here $c^{\prime}$ is independent of $I$. Since $\left(1-\left|z_{I}\right|\right) \sim$ $\left(1-\left|w_{I}\right|\right)$, with a constant independent of $I$, we conclude that

$$
\operatorname{dist}\left(z_{I}, \partial D\right) \geq c\left(1-\left|w_{I}\right|\right),
$$

where $c$ is independent of $I$.

To estimate diam $(D)$, it is sufficient to show that

$$
\operatorname{dist}(x, \widehat{I}) \leq c\left(1-\left|w_{I}\right|\right)
$$

for all $x \in \partial D$. For if $x$ and $y$ are in $\partial D$, then

$$
|x-y| \leq \operatorname{dist}(x, \widehat{I})+\operatorname{dist}(y, \widehat{I})+|\widehat{I}|,
$$


and we saw in Section 10 that $|\widehat{I}| \sim 1-\left|w_{I}\right|$. We showed (13.11) for $x \in \widetilde{I}$, in the proof of Lemma 10.5. We prove (13.11) for $x \in \gamma_{1}$; the same argument works for $x \in \gamma_{2}$.

Let $a$ and $\widehat{a}$ be the endpoints of $\gamma_{1}$ which lie in $I$ and $\widehat{I}$ respectively. Using the fact that $\gamma_{1}$ is chord-arc,

$$
\begin{aligned}
\operatorname{dist}(x, \widehat{I}) & \leq|x-\widehat{a}| \\
& \leq \ell_{\gamma_{1}}(x, \widehat{a}) \\
& \leq \ell_{\gamma_{1}}(a, \widehat{a}) \\
& \leq c|a-\widehat{a}| \\
& \leq|I|+|\widehat{I}|+\operatorname{dist}(I, \widehat{I}) .
\end{aligned}
$$

Now $|I| \leq c\left(1-\left|w_{I}\right|\right)$ by Lemma $8.1 ;|\widehat{I}| \leq c|I|$ by Lemma 10.3 , and $\operatorname{dist}(I, \widehat{I}) \leq$ $c\left(1-\left|w_{I}\right|\right)$ by the proof of Lemma 10.5. So we have established (13.11), which together with (13.1) proves Lemma 13.3.

Proof of Lemma 13.1. By Lemma 13.2, Lemma 13.3, and the remarks after the statement of Lemma 13.1, arclength on $\partial D$ and harmonic measure at $z_{I}$ in $D$ are $A_{\infty}$-equivalent with constants depending only on the chord-arc constant of $\Lambda_{J}$ and the constant $C$ from Lemma 13.3. Let $c_{1}$ and $\alpha_{1}$ be such that

$$
\frac{|E|}{|S|} \leq c_{1}\left[\frac{\omega\left(z_{I}, E, D\right)}{\omega\left(z_{I}, S, D\right)}\right]^{\alpha_{1}}
$$

for all Borel subsets $E$ of segments $S$ of $\partial D$. The leaves $\Lambda_{J}$ are also chord-arc, and $d_{\text {hyp }}\left(z_{J}, \partial \Lambda_{J}\right) \geq c>0$, so we may choose $c_{2}$ and $\alpha_{2}$ independent of $I$ such that

$$
\frac{\omega\left(z_{J}, E, \Lambda_{J}\right)}{\omega\left(z_{J}, S, \Lambda_{J}\right)} \leq c_{2}\left[\frac{|E|}{|S|}\right]^{\alpha_{2}}
$$

for all Borel subsets $E$ of segments $S$ of $\partial \Lambda_{J}$.

By the distortion estimates in Section 10, $|\widetilde{I}| \sim|I|$ and $|B| \sim|\widehat{B}|$. We use (13.14) and 13.15 on the sets $\widehat{B} \subset \widetilde{I} \subset \partial D$ and $\pi(\widehat{B}) \subset E_{J} \subset \partial \Lambda_{J}$ :

$$
\begin{aligned}
\frac{|B|}{|I|} & \leq c \frac{|\widehat{B}|}{|\widetilde{I}|} \\
& \leq c c_{1}\left[\frac{\omega\left(z_{I}, \widehat{B}, D\right)}{\omega\left(z_{I}, \widetilde{I}, D\right)}\right]^{\alpha_{1}} \\
& =c c_{1}\left[\frac{\omega\left(\pi\left(z_{I}\right), \pi(\widehat{B}), \pi(D)\right)}{\omega\left(\pi\left(z_{I}\right), \pi(\widetilde{I}), \pi(D)\right)}\right]^{\alpha_{1}} \\
& =c c_{1}\left[\frac{\omega\left(z_{J}, \pi(\widehat{B}), \Lambda_{J}\right)}{\omega\left(z_{J}, E_{J}, \Lambda_{J}\right)}\right]^{\alpha_{1}} \\
& \leq c c_{1}\left[c_{2}\left(\frac{|\pi(\widehat{B})|}{\left|E_{J}\right|}\right)^{\alpha_{2}}\right]^{\alpha_{1}},
\end{aligned}
$$

which establishes the right-hand inequality in (13.1). A similar argument, again using (13.3), proves the left-hand inequality in (13.1). The constants are independent of $I$ and $B$, but they depend on the $N$ such that $\left|E_{J}\right|=2 \cdot 3^{N}|J|$, since the chord-arc constant of $\Lambda_{J}$ depends on this $N$. 
Lemma 13.4. There are positive constants $c_{1}$ and $c_{2}$ such that for all $n \geq 1$, for every standard grid interval $I \in \mathcal{H}_{n-1}$,

$$
E X_{i}=\frac{1}{\mu(I)} \int_{I} X_{i}(x) d \mu \geq c_{1}
$$

and

$$
E X_{i}^{2}=\frac{1}{\mu(I)} \int_{I} X_{i}^{2}(x) d \mu \leq c_{2} .
$$

Here $X_{i}(x)=\log _{3}\left(\left|J_{i}(x)\right| /|J|\right)$ is the auxiliary function for a particle which makes its $i^{\text {th }}$ jump from $J=\pi(\widehat{I})$ and which has not yet reached $J_{\infty}$.

Proof. Let $L$ be the component of $[0,1] \backslash K$ which contains $J=\pi(\widehat{I})$.

We begin by slightly modifying some definitions, in order to simplify the calculations. In each component $L^{\prime}$ of $E_{J} \backslash K$, we amalgamated the $2 N$ Whitney intervals in the centre of $L^{\prime}$ into a single interval $J_{c}=J_{-N} \cup \cdots \cup J_{N}$. We now temporarily assume that we have retained the individual intervals $J_{-N}, \ldots, J_{N}$ instead. We also change the definitions of the region $R_{3}$ and of the function $F_{n}$. Set $S_{3}=J_{-1} \cup J_{1}$ in the component $L^{\prime}$ of $[0,1] \backslash K$ which is the left half of $E_{J}$, and let $R_{3}=P \circ \pi^{-1}\left(S_{3}\right)$. (In Section 11, we had $S_{3}=J_{-N} \cup \cdots \cup J_{N} \subset L^{\prime}$. The regions $R_{1}$ and $R_{5}$ are unchanged; the regions $R_{2}$ and $R_{4}$ become larger since they now include $J_{-N} \cup \cdots \cup J_{-2}$ and $J_{2} \cup \cdots \cup J_{N}$ respectively.)

Define

$$
F_{n}(x)= \begin{cases}1, & x \in R_{1} \cup R_{5} ; \\ (1-\varepsilon) \frac{|I|}{\left|R_{3}\right|}, & x \in R_{3} ; \\ \delta, & x \in R_{2} \cup R_{4},\end{cases}
$$

using the new definition of $R_{3}$. (At the end of the proof we show that the estimates with these new definitions imply the estimates for the original functions $F_{n}$ and $X_{i}$.)

With these assumptions, we begin with (13.17). Let $I \in \mathcal{H}_{n-1}$ be a standard interval. Let $J=\pi(\widehat{I})$. For each point $x$ in $I$, let $I_{i}(x)$ be the interval in $\mathcal{H}_{n}$ which contains $x$. I, n, and $i$ are fixed throughout the proof. Let $J_{i}(x)=\pi\left(\widehat{I}_{i}(x)\right)$. Then

$$
X_{i}(x)=\log _{3}\left[\frac{\left|J_{i}(x)\right|}{|J|}\right] .
$$

Notice that to compute $E X_{i}$ or $E X_{i}^{2}$, we need only deal with $F_{n}$. The functions $F_{l}$ for $l \neq n$ are irrelevant, since for $l<n F_{l}$ is constant on $I$, and for $l>n F_{l}$ has mean value one on each subset of $I$ where $X_{i}$ is constant.

Let

$$
\begin{aligned}
B_{k} & =\left\{x \in I \mid X_{i}(x)=N-k\right\} \\
& =\left\{x \in I|| J_{i}(x)\left|=3^{N-k}\right| J \mid\right\} \\
& =\left\{x \in I|| J_{i}(x)\left|=2^{-1} \cdot 3^{-k}\right| E_{J} \mid\right\},
\end{aligned}
$$

for $k \geq 1$. These $B_{k}$ 's are exactly the sets on which the integrand $X_{i}$ in (13.17) is constant. The union of the $B_{k}$ 's is $I$. We estimate (13.17) by integrating over each $B_{k}$ separately (see (13.27) below); before that we need some preliminaries.

Let $\widehat{B_{k}}$ be the subset of $\widetilde{I}$ such that $P\left(\widehat{B_{k}}\right)=B_{k}$. Then $\pi\left(\widehat{B_{k}}\right)$ is the union of all Whitney intervals in $E_{J}$ of length $2^{-1} \cdot 3^{-k}\left|E_{J}\right|$. 
We count the number of Whitney intervals of this length in $E_{J}$. The segment $E_{J}$ contains one component of $\overline{\mathbb{R}} \backslash K$ of length $2^{-1}\left|E_{J}\right|$, and it contains $2^{j}$ components of $\overline{\mathbb{R}} \backslash K$ of length $2^{-1} \cdot 3^{-j-1}\left|E_{J}\right|$, for each $j \geq 0$. Within each component $L$ of $\overline{\mathbb{R}} \backslash K$ there are two Whitney intervals of size $3^{-l}|L|$, for each $l \geq 1$. Therefore $E_{J}$ contains $2^{k}$ Whitney intervals of length $2^{-1} \cdot 3^{-k}\left|E_{J}\right|=3^{N-k}|J|$, for each $k \geq 1$. Hence

$$
\left|\pi\left(\widehat{B_{k}}\right)\right|=2^{k} \cdot 2^{-1} \cdot 3^{-k}\left|E_{J}\right|=\frac{1}{2}\left(\frac{2}{3}\right)^{k}\left|E_{J}\right|,
$$

for $k \geq 1$.

Therefore, by Lemma 13.1, there are positive constants $c$ and $\alpha$, independent of $I$ but dependent on $N$, such that

$$
\frac{\left|B_{k}\right|}{|I|} \leq c\left[\frac{\left|\pi\left(\widehat{B_{k}}\right)\right|}{\left|E_{J}\right|}\right]^{\alpha}=c\left[\frac{1}{2}\left(\frac{2}{3}\right)^{k}\right]^{\alpha}=c \beta^{k},
$$

where $\beta=(2 / 3)^{\alpha}$ is strictly less than one.

We also estimate the sizes of $B_{k} \cap R_{1}$ and $B_{k} \cap R_{5}$, for $k \geq 1$. The sets $R_{j}$ and $S_{j}$, $1 \leq j \leq 5$, were defined in Section 11. $S_{1}=\pi\left(\widehat{R_{1}}\right)$ is the segment at one end of $E_{J}$, contained in $E_{J} \backslash K$, such that $\left|S_{1}\right|=\frac{3}{2} \cdot 3^{-Q}|J|$. Q is a large integer, independent of $I$. The largest Whitney interval in $S_{1}$ has length $3^{-Q}|J|=2^{-1} \cdot 3^{-Q-N}\left|E_{J}\right|$. The segment $S_{1}$ contains exactly one Whitney interval of length $2^{-1} \cdot 3^{-k}\left|E_{J}\right|$, in other words one Whitney interval which lies in $\pi\left(\widehat{B_{k}}\right)$, for each $k \geq Q+N$. By Lemma 13.1,

$$
\begin{aligned}
\frac{\left|B_{k} \cap R_{1}\right|}{|I|} & \leq c\left[\frac{\left|\pi\left(\widehat{B_{k}} \cap \widehat{R_{1}}\right)\right|}{\left|E_{J}\right|}\right]^{\alpha} \\
& =c\left[\frac{\left|\pi\left(\widehat{B_{k}}\right) \cap S_{1}\right|}{\left|E_{J}\right|}\right]^{\alpha} \\
& =c\left[\frac{2^{-1} \cdot 3^{-k}\left|E_{J}\right|}{\left|E_{J}\right|}\right]^{\alpha} \\
& =c\left(3^{-\alpha}\right)^{k},
\end{aligned}
$$

for $k \geq Q+N$. The left-hand side is zero when $0 \leq k \leq Q+N-1$.

$S_{5}=\pi\left(\widehat{R_{5}}\right)$ is the segment at the other end of $E_{J}$ such that $\left|S_{5}\right|=3^{-Q}|J| . S_{5}$ is a closed construction interval of the Cantor set $K$. The largest Whitney intervals in $S_{5}$ are two intervals of length $3^{-2} \cdot 3^{-Q}|J|=2^{-1} \cdot 3^{-Q-N-2}\left|E_{J}\right| . S_{5}$ contains $2^{k-Q-N-1}$ Whitney intervals of size $2^{-1} \cdot 3^{-k}\left|E_{J}\right|$, for each $k \geq Q+N+2$. By Lemma 13.1,

$$
\begin{aligned}
\frac{\left|B_{k} \cap R_{5}\right|}{|I|} & \leq c\left[\frac{\left|\pi\left(\widehat{B_{k}}\right) \cap S_{5}\right|}{\left|E_{J}\right|}\right]^{\alpha} \\
& =c\left[\frac{2^{k-Q-N-1} \cdot 2^{-1} \cdot 3^{-k}\left|E_{J}\right|}{\left|E_{J}\right|}\right]^{\alpha} \\
& =c\left(2^{-Q-N}\right)^{\alpha}(2 / 3)^{k \alpha} \\
& =c\left(2^{-\alpha}\right)^{Q+N} \beta^{k},
\end{aligned}
$$

for $k \geq Q+N+2$. The left-hand side vanishes for $0 \leq k \leq Q+N+1$. 
The set $B_{1}$ is exactly $R_{3}$, and so

$$
F_{n} \equiv(1-\varepsilon)|I| /\left|B_{1}\right| \quad \text { on } B_{1} .
$$

Also, on $I, \mu_{n-1}$ is given by

$$
d \mu_{n-1}=\left(\mu_{n-1}(I) /|I|\right) d x .
$$

Therefore

$$
\begin{aligned}
\mu\left(B_{1}\right) & =\mu_{n}\left(B_{1}\right) \\
& =\int_{B_{1}} F_{n}(x) d \mu_{n-1}(x) \\
& =(1-\varepsilon) \frac{|I|}{\left|B_{1}\right|} \frac{\mu_{n-1}(I)}{|I|}\left|B_{1}\right| \\
& =(1-\varepsilon) \mu_{n-1}(I) \\
& =(1-\varepsilon) \mu(I) .
\end{aligned}
$$

After these preliminaries we can estimate $E X_{i}$ :

$$
\begin{aligned}
E X_{i} & =\frac{1}{\mu(I)} \int_{I} \log _{3}\left[\frac{\left|J_{i}(x)\right|}{|J|}\right] d \mu(x) \\
& =\sum_{k=1}^{\infty}(N-k) \frac{\mu\left(B_{k}\right)}{\mu(I)} \\
& =(N-1) \frac{\mu\left(B_{1}\right)}{\mu(I)}+\sum_{k=2}^{\infty}(N-k) \frac{\mu\left(B_{k}\right)}{\mu(I)} \\
& \geq(N-1)(1-\varepsilon)+\sum_{k=N}^{\infty}(N-k) \frac{\mu\left(B_{k}\right)}{\mu(I)} \\
& \geq(N-1)(1-\varepsilon)-\sum_{k=N}^{\infty} k \frac{\mu\left(B_{k}\right)}{\mu(I)} \\
& =(N-1)(1-\varepsilon)-\sum_{k=N}^{\infty} k \frac{\mu_{n}\left(B_{k}\right)}{\mu_{n-1}(I)} \\
& \geq(N-1)(1-\varepsilon)-\sum_{k=N}^{\infty} \frac{k}{\mu_{n-1}(I)} \int_{B_{k}} F_{n}(x) d \mu_{n-1}(x) .
\end{aligned}
$$

The last series converges. To show this, we split each $B_{k}$ into three pieces, $B_{k} \cap$ $\left(R_{2} \cup R_{4}\right), B_{k} \cap R_{1}$, and $B_{k} \cap R_{5}$, and estimate the sums over the three types of terms. 
First, since $F_{n} \equiv \delta$ on $R_{2} \cup R_{4}$,

$$
\begin{aligned}
\sum_{k=N}^{\infty} \frac{k}{\mu_{n-1}(I)} \int_{B_{k} \cap\left(R_{2} \cup R_{4}\right)} & F_{n}(x) d \mu_{n-1}(x) \\
& =\sum_{k=N}^{\infty} k \delta \frac{\mu_{n-1}\left(B_{k} \cap\left(R_{2} \cup R_{4}\right)\right)}{\mu_{n-1}(I)} \\
& =\delta \sum_{k=N}^{\infty} k \frac{\left|B_{k} \cap\left(R_{2} \cup R_{4}\right)\right|}{|I|} \\
& \leq \delta \sum_{k=N}^{\infty} k \frac{\left|B_{k}\right|}{|I|} \\
& \leq \delta c(N) \sum_{k=N}^{\infty} k \beta^{k} \\
& \leq \delta \frac{c(N)}{(1-\beta)^{2}}
\end{aligned}
$$

which can be made arbitrarily small by choosing $\varepsilon$ small (which implies $\delta$ small) in the definition of $F_{n}$. Here the second equality holds because, on $I, \mu_{n-1}$ is a constant multiple of Lebesgue measure, and the second last line holds by (13.23).

Similarly, since $F_{n} \equiv 1$ on $R_{1}$,

$$
\begin{aligned}
\sum_{k=N}^{\infty} \frac{k}{\mu_{n-1}(I)} \int_{B_{k} \cap R_{1}} & F_{n}(x) d \mu_{n-1}(x) \\
& =\sum_{k=N}^{\infty} k \frac{\mu_{n-1}\left(B_{k} \cap R_{1}\right)}{\mu_{n-1}(I)} \\
& =\sum_{k=N}^{\infty} k \frac{\left|B_{k} \cap R_{1}\right|}{|I|} \\
& =\sum_{k=N+Q}^{\infty} k \frac{\left|B_{k} \cap R_{1}\right|}{|I|} \\
& \leq c(N) \sum_{k=N+Q}^{\infty} k\left(3^{-\alpha}\right)^{k}
\end{aligned}
$$

which can be made arbitrarily small by choosing $Q$ large. Here the last line holds by (13.24). 
Finally, since $F_{n} \equiv 1$ on $R_{5}$,

$$
\begin{aligned}
\sum_{k=N}^{\infty} \frac{k}{\mu_{n-1}(I)} \int_{B_{k} \cap R_{5}} & F_{n}(x) d \mu_{n-1}(x) \\
& =\sum_{k=N}^{\infty} k \frac{\mu_{n-1}\left(B_{k} \cap R_{5}\right)}{\mu_{n-1}(I)} \\
& =\sum_{k=N}^{\infty} k \frac{\left|B_{k} \cap R_{5}\right|}{|I|} \\
& =\sum_{k=Q+N+2}^{\infty} k \frac{\left|B_{k} \cap R_{5}\right|}{|I|} \\
& \leq c(N)\left(2^{-\alpha}\right)^{Q+N} \sum_{k=Q+N+2}^{\infty} k \beta^{k} \\
& \leq \frac{c(N)\left(2^{-\alpha}\right)^{Q+N}}{(1-\beta)^{2}},
\end{aligned}
$$

which decreases to zero as $Q$ increases. Here the second last inequality holds by $(13.25)$.

Once $N$ is fixed, we can make (13.28), (13.29), and (13.30) arbitrarily small by choosing $\varepsilon$ sufficiently small and $Q$ sufficiently large. Therefore we can make the last term in (13.27) arbitrarily small. So for some positive constant $c_{1}$ depending on $N$, we can ensure that $E X_{i} \geq c_{1}$ for all standard intervals $I$ in $\mathcal{H}_{n-1}$, for all $n \geq 1$.

We can estimate the second moment more simply. We showed in Section 11 that there is an upper bound $1 / \delta_{0}$ for $F_{n}$ on $I$, independent of $I$ and $n$. Then

$$
\begin{aligned}
E X_{i}^{2} & =\frac{1}{\mu(I)} \int_{I}\left\{\log _{3}\left[\frac{\left|J_{i}(x)\right|}{|J|}\right]\right\}^{2} d \mu(x) \\
& =\sum_{k=1}^{\infty}(N-k)^{2} \frac{\mu\left(B_{k}\right)}{\mu(I)}=\sum_{k=1}^{\infty}(N-k)^{2} \frac{\mu_{n}\left(B_{k}\right)}{\mu_{n-1}(I)} \\
& =\sum_{k=1}^{\infty}(N-k)^{2} \frac{1}{\mu(I)} \int_{B_{k}} F_{n}(x) d \mu_{n-1}(x) \\
& \leq \frac{1}{\delta_{0}} \sum_{k=1}^{\infty}(N-k)^{2} \frac{\mu_{n-1}\left(B_{k}\right)}{\mu_{n-1}(I)} \\
& =\frac{1}{\delta_{0}} \sum_{k=1}^{\infty}(N-k)^{2} \frac{\left|B_{k}\right|}{|I|} \leq \frac{1}{\delta_{0}} \sum_{k=1}^{\infty}\left(N^{2}+k^{2}\right) \frac{\left|B_{k}\right|}{|I|} \\
& \leq \frac{c(N)}{\delta_{0}} \sum_{k=1}^{\infty}\left(N^{2}+k^{2}\right) \beta^{k} \\
& \leq \frac{c(N)}{\delta_{0}}\left[\frac{N^{2}}{(1-\beta)}+\frac{1}{(1-\beta)^{3}}\right]
\end{aligned}
$$


using (13.23) in the second last line. In the fifth line we used the fact that $\mu_{n-1}$ is a constant multiple of Lebesgue measure on $I$. Again, once $N, Q$, and $\varepsilon$ are fixed, this estimate gives a uniform upper bound $c_{2}$ on $E X_{i}^{2}$ for all standard intervals $I \in \mathcal{H}_{n-1}$, for all $n \geq 1$.

It remains to prove the same estimates for our original functions $X_{i}$ and $F_{n}$. Write $Y_{i}$ and $G_{n}$ for the modified versions of $X_{i}$ and $F_{n}$ defined at the start of the proof and used in the calculations (13.19)-(13.31) above.

To estimate $E X_{i}^{2}$, define $Z_{i}(x)=N^{2}+k^{2}$ on $B_{k}, k \geq 1$, where $B_{k}=\{x \in I \mid$ $\left.Y_{i}(x)=N-k\right\}$ as above. A comparison of $Y_{i}, X_{i}$, and $Z_{i}$ shows that on each $B_{k}$,

$$
X_{i}^{2} \leq(N+k)^{2} \leq 2\left(N^{2}+k^{2}\right)=2 Z_{i} .
$$

The calculation (13.31) shows that the mean value of $Z_{i} G_{n}$ on $I$ is at most $c_{2}$. Also, $F_{n} \sim G_{n}$ with constants independent of $I$, since they are both bounded above and below by positive constants independent of $I$. Therefore

$$
\begin{aligned}
E X_{i}^{2} & =\frac{1}{|I|} \int_{I} X_{i}^{2} F_{n} d x \\
& \leq c \frac{1}{|I|} \int_{I} 2 Z_{i} G_{n} d x \\
& \leq 2 c c_{2},
\end{aligned}
$$

for all $I$.

Finally, we estimate $E X_{i}$. Let $G_{b}$ (respectively $F_{b}$ ) be the maximum value of $G_{n}$ (respectively $F_{n}$ ). Then $G_{b} \sim F_{b}$ with constants independent of $I$, by a comparison of harmonic measures in $\Lambda_{J}$. Let

$$
I_{+}=\left\{x \in I \mid F_{n}(x)=F_{b}\right\}
$$

and

$$
I_{s}=\left\{x \in I \mid F_{n}(x)=\delta^{\prime}\right\},
$$

where $\delta^{\prime}$ is the minimum value of $F_{n}$ on $I$. In the next calculation we neglect the region where $Y_{i} \equiv 1$. Then, since $X_{i} \geq Y_{i}$ on $I$,

$$
\begin{aligned}
E X_{i} & =\frac{1}{|I|} \int_{I} X_{i} F_{n} d x \\
& \geq \frac{1}{|I|} \int_{I} Y_{i} F_{n} d x \\
& \geq \frac{1}{|I|} \int_{I_{+}} Y_{i} F_{b} d x-\frac{1}{|I|} \int_{I_{s}} Y_{i} F_{n} d x \\
& \geq c \frac{1}{|I|} \int_{I_{+}} Y_{i} G_{b} d x-\frac{1}{|I|} \int_{I_{s}} Y_{i} F_{n} d x \\
& \geq c \frac{N-1}{2}-\delta^{\prime} \frac{1}{|I|} \int_{I_{s}} Y_{i} d x ;
\end{aligned}
$$

by (13.27). We showed above that $\frac{1}{|I|} \int_{I_{s}} Y_{i} d x$ is bounded above by a constant independent of $I$. Therefore, by choosing $\delta^{\prime}$ small enough in the definition of $F_{n}$, we may ensure that $E X_{i}$ is bounded below by a positive constant independent of $I$.

This completes the proof of Lemma 13.4. 


\section{Estimates $E X_{i} \geq c_{1}$ AND $E X_{i}^{2} \leq c_{2}$ FOR NON-STANDARD INTERVAls}

Let $I \in \mathcal{H}$ be any non-standard grid interval such that $J_{c}=\pi(\widehat{I})$ is the nonstandard central interval in some component $L$ of $[0,1] \backslash K$. In this section we prove (Lemma 14.3) the estimates $\mu(I)^{-1} \int_{I} X_{i} d \mu \geq c_{1}$ and $\mu(I)^{-1} \int_{I} X_{i}^{2} d \mu \leq c_{2}$, where $c_{1}$ and $c_{2}$ are positive constants independent of $I$. Here $X_{i}(x)=\log _{3}\left(\left|J_{i}(x)\right| /\left|J_{c}\right|\right)$ is the auxiliary function for a particle which makes its $i^{\text {th }}$ jump from $J_{c}=\pi(\widehat{I})$ and which has not yet reached $J_{\infty}$. We begin with an estimate, analogous to Lemma 13.1, on the distortion in length caused by the map $P \circ \pi^{-1}$.

Lemma 14.1. Let $I \in \mathcal{H}_{n-1}, n \geq 1$, be a non-standard grid interval such that $J_{c}=\pi(\widehat{I})$ is the central interval in some component $L$ of $[0,1] \backslash K$. Fix a number $\lambda>1$, and let $q$ be any positive integer. Let $B$ and $T$ be unions of grid intervals from $\mathcal{H}_{n}$ such that $B \subset T \subset I$ and $\pi(\widehat{T}) \subset\left\{w \mid \lambda^{q} \leq \operatorname{dist}\left(w, J_{c}\right) \leq \lambda^{q+1}\right\}$. There are constants $c$ and $\alpha$, independent of $q, B, T, I$, and $n$, such that

$$
\frac{|B|}{|T|} \leq c\left[\frac{|\pi(\widehat{B})|}{|\pi(\widehat{T})|}\right]^{\alpha}
$$

The proof is essentially the same as for standard intervals, but we need a stronger version of $A_{\infty}$-equivalence because $\partial \Lambda_{J}$ is unbounded. Namely, if $\Omega$ is a chord-arc domain, and $z \in \Omega$, then there are positive constants $c_{1}, c_{2}, \alpha_{1}$, and $\alpha_{2}$ such that

$$
\frac{|E|}{|S|} \leq c_{1}\left[\frac{\omega(z, E, \Omega)}{\omega(z, S, \Omega)}\right]^{\alpha_{1}} \quad \text { and } \quad \frac{\omega(z, E, \Omega)}{\omega(z, S, \Omega)} \leq c_{2}\left[\frac{\mid E}{|S|}\right]^{\alpha_{2}}
$$

whenever $S$ is a segment of $\partial \Omega$ satisfying

$$
S \subset\left\{w \mid \lambda^{q} \leq \operatorname{dist}(w, z) \leq \lambda^{q+1}\right\}
$$

for any positive integer $q$ and for a fixed $\lambda>1$, and $E$ is a Borel subset of $S$. The constants $c_{1}, c_{2}, \alpha_{1}$, and $\alpha_{2}$ depend on the chord-arc constant of $\partial \Omega$ and on the constant $\lambda$, but not on $q$.

This follows from the case of bounded chord-arc domains. By dilations and translations we may assume that $z=i$, and that $-i$ is at least distance $\varepsilon$ from $\Omega$. Consider the Möbius transformation $\tau: w \mapsto(w+i)^{-1}$. This maps $\Omega$ to a bounded chord-arc domain. Harmonic measure is invariant under Möbius transformations. Also, for each positive integer $q$, the map $\tau$ scales everything in the annulus in (14.3), in particular $E$ and $S$, by approximately the same factor. The estimates in (14.2) follow.

Consider the domain $D=\pi^{-1}\left(\Lambda_{J_{c}}\right)$, where $I$ is a non-standard interval as above. The analogues of Lemmas 13.2 and 13.3 hold, and Lemma 14.1 follows in the same way as Lemma 13.1 follows from Lemmas 13.2 and 13.3.

Notice that, although the interval $I$ is no longer standard, arclength and harmonic measure are still $A_{\infty}$-equivalent on $\partial D$, since $D$ is a bounded chord-arc domain. Therefore, for any segment $S$ of $\partial D$ and Borel subset $E$ of $S$, we still have

$$
\frac{|E|}{|S|} \leq c_{1}\left[\frac{\omega\left(z_{J}, \pi(E), \Lambda_{J_{c}}\right)}{\omega\left(z_{J}, \pi(S), \Lambda_{J_{c}}\right)}\right]^{\alpha_{1}},
$$

and the analogous inequality in the other direction, both with constants independent of $I$. We will frequently use this fact, combined with direct estimates of harmonic measures in $\Lambda_{J_{c}}$. We refer to such an argument as a comparison of harmonic measure on the domain side. 

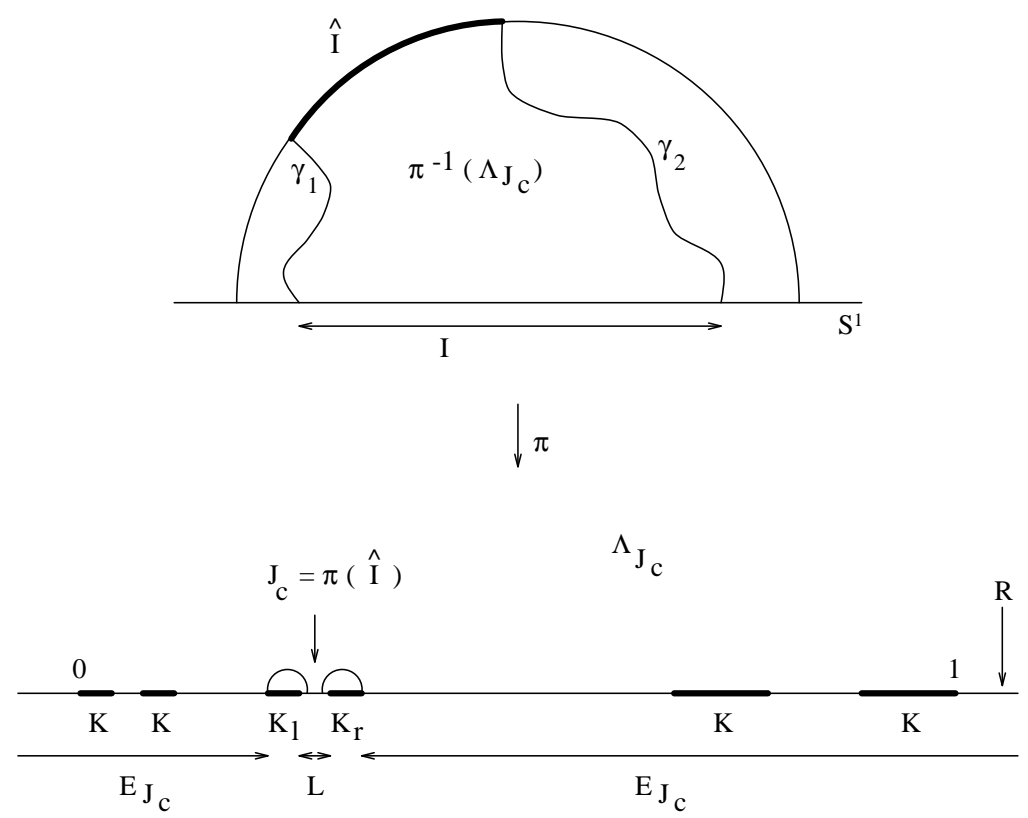

Figure 13. A non-standard central interval $J_{c}$.

We will need the next lemma, which follows immediately from our decomposition of $\overline{\mathbb{R}} \backslash K$ into Whitney intervals.

Lemma 14.2. Let $A$ be a subinterval of $[0,1]$ of length $3^{-l}, l \geq 0$. Then

1. A contains a Whitney interval of length at least $3^{-2}|A|$; and

2. A meets at most $2^{k}$ Whitney intervals of length $3^{-k}|A|$, for $k \geq 1$.

The main result of this section is:

Lemma 14.3. There are positive constants $c_{1}$ and $c_{2}$ such that for all $n \geq 1$, for every non-standard grid interval $I \in \mathcal{H}_{n-1}$ such that $\pi(\widehat{I})=J_{c}$ is the central Whitney interval in some component $L$ of $[0,1] \backslash K$,

$$
E X_{i}=\frac{1}{\mu(I)} \int_{I} X_{i}(x) d \mu \geq c_{1}
$$

and

$$
E X_{i}^{2}=\frac{1}{\mu(I)} \int_{I} X_{i}^{2}(x) d \mu \leq c_{2} .
$$

Here $X_{i}(x)=\log _{3}\left(\left|J_{i}(x)\right| /\left|J_{c}\right|\right)$ is the auxiliary function for a particle which has not yet reached $J_{\infty}$ and which makes its $i^{\text {th }}$ jump from $J_{c}=\pi(\widehat{I})$.

Proof. Let $J_{c}$ be the central Whitney interval, $J_{c}=J_{-N} \cup \cdots \cup J_{N}$, in some component $L$ of $[0,1] \backslash K$. Make the convention that $\left|J_{c}\right|=|L| / 3$. The tips of the leaves for the standard intervals in $L$ cover $K_{l}$ and $K_{r}$, the closed construction intervals of $K$ immediately to the left and right of $L$. So the tip $E_{J_{c}}$ of the leaf for $J_{c}$ is $\overline{\mathbb{R}} \backslash\left(K_{l} \cup L \cup K_{r}\right)$. See Figure 13 .

To simplify the calculations, we again assume, as in the proof of Lemma 13.4, that in each component $L$ of $E_{J_{c}} \backslash K$ we have not amalgamated the $2 N$ central 
Whitney intervals but have retained them individually. As before, the result follows from this simplified version.

We divide $E_{J_{c}}$ into subsets $V_{j}, j \geq 1$, of length $\left|V_{j}\right|=3^{j+10}\left|J_{c}\right|$. Let each $V_{j}$ have two components of equal length, one on each side of $J_{c}$. Put the components of $V_{1}$ as close as possible to $J_{c}$, that is, on each side of and adjacent to $K_{l} \cup L \cup K_{r}$. Put the components of $V_{2}$ on each side of and adjacent to $V_{1} \cup K_{l} \cup L \cup K_{r}$, and so on. For each $j \geq 1$, let $T_{j}$ be the subset of $I$ such that $\pi\left(\widehat{T_{j}}\right)=V_{j}$.

Fix $j \geq 1$, and for each $k \geq 1$ define the subset $B_{k}$ of $T_{j}$ by

$$
\begin{aligned}
B_{k} & =\left\{x \in T_{j}|| J_{i}(x)\left|=3^{-k}\right| V_{j} \mid\right\} \\
& =\left\{x \in T_{j}|| J_{i}(x)\left|=3^{-k} \cdot 3^{j+10}\right| J_{c} \mid\right\} \\
& =\left\{x \in T_{j} \mid X_{i}(x)=j+10-k\right\} .
\end{aligned}
$$

By Lemma 14.2 , the set $\pi\left(\widehat{B_{k}}\right)$ meets at most $2^{k}$ Whitney intervals of size $3^{-k}\left|V_{j}\right|$, so

$$
\left|\pi\left(\widehat{B_{k}}\right)\right| \leq\left(\frac{2}{3}\right)^{k}\left|V_{j}\right|
$$

By Lemma 14.1,

$$
\frac{\left|B_{k}\right|}{\left|T_{j}\right|} \leq c\left[\frac{\left|\pi\left(\widehat{B_{k}}\right)\right|}{\left|V_{j}\right|}\right]^{\alpha}=c\left[\left(\frac{2}{3}\right)^{k}\right]^{\alpha}=c \beta^{k}
$$

where $\beta=(2 / 3)^{\alpha}$ is strictly less than one, and $c$ and $\beta$ are independent of $j$.

In fact, Lemma 14.1 in the form stated above may not apply, since $B_{k}$ and $T_{j}$ need not be unions of whole grid intervals. However, the difficulty is only that at either end of $T_{j}$ there may be grid intervals which meet $T_{j}$ (and hence some $B_{k}$ ) but are not contained in $T_{j}$ (or $B_{k}$ ). The distortion estimates in Section 10, and the fact that grid intervals are comparable in size to their neighbours, imply that Lemma 14.1 can be extended to cover this case.

We first prove the estimate on $E X_{i}^{2}$. In estimating $X_{i}^{2}$ we can neglect the function $F_{n}$, since the $F_{n}$ we use will be bounded above by a constant independent of $I$. So it is enough to find a bound independent of $I$ for

$$
\frac{1}{|I|} \int_{I} X_{i}^{2} d x=\sum_{j=1}^{\infty} \frac{\left|T_{j}\right|}{|I|} \frac{1}{\left|T_{j}\right|} \int_{T_{j}}\left\{\log _{3}\left[\frac{\left|J_{i}(x)\right|}{\left|J_{c}\right|}\right]\right\}^{2} d x
$$

First, for each $j$,

$$
\begin{aligned}
\frac{1}{\left|T_{j}\right|} \int_{T_{j}}\left\{\log _{3}\left[\frac{\left|J_{i}(x)\right|}{\left|J_{c}\right|}\right]\right\}^{2} & =\sum_{k=1}^{\infty}\{j+10-k\}^{2} \frac{\left|B_{k}\right|}{\left|T_{j}\right|} \\
& \leq c \sum_{k=1}^{\infty}\left\{j^{2}+(k-10)^{2}\right\} \beta^{k} \\
& \leq c^{\prime} j^{2}+c^{\prime \prime} .
\end{aligned}
$$


Also, $\left|T_{j}\right| /|I|$ decays exponentially in $j$ :

$$
\begin{aligned}
\frac{\left|T_{j}\right|}{|I|} & \leq c \frac{\left|\widehat{T_{j}}\right|}{|\widetilde{I}|} \\
& \leq c c_{1}\left[\frac{\omega\left(z_{I}, \widehat{T_{j}}, \pi^{-1}\left(\Lambda_{J_{c}}\right)\right)}{\omega\left(z_{I}, \widetilde{I}, \pi^{-1}\left(\Lambda_{J_{c}}\right)\right)}\right]^{\alpha_{1}} \\
& =c c_{1}\left[\frac{\omega\left(z_{J}, V_{j}, \Lambda_{J_{c}}\right)}{\omega\left(z_{J}, E_{J_{c}}, \Lambda_{J_{c}}\right)}\right]^{\alpha_{1}} \\
& \leq c\left[\omega\left(z_{J}, V_{j}, \mathbf{U}\right)\right]^{\alpha_{1}},
\end{aligned}
$$

with constants independent of $j$. Now $\pi \cdot \omega\left(z_{J}, V_{j}, \mathbf{U}\right)$ is the angle subtended at $z_{J}$ by $V_{j}$. By elementary trigonometry, this angle is less than $c 3^{-j}$, with $c$ independent of $j$. Therefore

$$
\frac{\left|T_{j}\right|}{|I|} \leq c 3^{-\alpha_{1} j}
$$

Hence

$$
\frac{1}{|I|} \int_{I} X_{i}^{2} d x \leq c \sum_{j=1}^{\infty} 3^{-\alpha_{1} j}\left(c^{\prime} j^{2}+c^{\prime \prime}\right) .
$$

The right-hand side is finite and independent of $I$.

It remains to prove the estimate on $E X_{i}$. Fix $\varepsilon>0$, and $j \geq 2$. By Lemma 14.2, $B_{2}$ is not empty. (For those $V_{j} \not \subset[0,1], B_{2}$ may be empty; part a) of Lemma 14.2 need not hold as stated because of the amalgamation of the large Whitney intervals far from $[0,1]$ to form $J_{\infty}$. However our argument can be modified to cover this case.) Let $B=B_{1} \cup B_{2}$, and define $F_{n}$ on $T_{j}$ by

$$
F(x)=F_{n}(x)= \begin{cases}(1-\varepsilon) \frac{\left|T_{j}\right|}{|B|}, & x \in B ; \\ \delta, & x \in T_{j} \backslash B\end{cases}
$$

where $\delta=\varepsilon\left|T_{j}\right| /\left|T_{j} \backslash B\right|$ is chosen so that $F$ has mean value one on $T_{j}$. A comparison of harmonic measure shows that $\left|T_{j}\right| /|B|$ is uniformly bounded for all $j$ and $I$. Therefore $F$ is uniformly bounded above and below by positive constants independent of $j$ and $I$.

On $B, X_{i} \geq j+8$. Then

$$
\begin{aligned}
\frac{1}{\mu\left(T_{j}\right)} \int_{T_{j}} X_{i} d \mu & =\frac{1}{\left|T_{j}\right|} \int_{T_{j}} X_{i} F d x \\
& \geq(j+8)(1-\varepsilon)+\delta \sum_{k=3}^{\infty}(j+10-k) \frac{\left|B_{k}\right|}{\left|T_{j}\right|} \\
& \geq(j+8)(1-\varepsilon)-\delta \sum_{k=j+10}^{\infty}(k-j-10) \frac{\left|B_{k}\right|}{\left|T_{j}\right|} \\
& \geq(j+8)(1-\varepsilon)-\delta c(N) \sum_{k-j=10}^{\infty}(k-j-10) \beta^{k} .
\end{aligned}
$$

The sum is finite and independent of $j$. Hence, by choosing $\delta$ (or equivalently $\varepsilon$ ) small enough in the definition of $F$, we may ensure that the mean of $X_{i} F$ with respect to $\mu$ on $T_{j}$ is at least some positive constant $c_{1}$, independent of $j$. 
To ensure that $F \equiv 1$ on suitable intervals at each end of $I$, we define $F$ slightly differently on $T_{1}$. Specifically, let $I_{l}$ and $I_{r}$ be subintervals at each end of $I$. Then $\pi\left(\widehat{I_{l}}\right)$ and $\pi\left(\widehat{I_{r}}\right)$ are subintervals of $V_{1}$, one on each side of $J_{c}$ and as close as possible to $J_{c}$; in other words, adjacent to $K_{l} \cup L \cup K_{r}$. Choose the lengths of $I_{l}$ and $I_{r}$ so that

$$
\frac{\left|\pi\left(\widehat{I_{l}}\right)\right|}{\left|T_{1}\right|}=\frac{\left|\pi\left(\widehat{I_{r}}\right)\right|}{\left|T_{1}\right|}=\frac{3}{2} \cdot 3^{-Q}
$$

for some large integer $Q$. The harmonic measure of $\pi\left(\widehat{I_{l}}\right) \cup \pi\left(\widehat{I_{r}}\right)$ in $\Lambda_{J_{c}}$ as seen from $z_{J}$ is a positive constant independent of $I$, since the domains $\Lambda_{J_{c}}$ for different $I$ are all Euclidean dilations of each other. By a comparison of harmonic measure, there is an $\eta>0$ such that $\left|I_{l}\right|$ and $\left|I_{r}\right| \geq \eta|I|$ for all $I$. Now let

$$
F(x)= \begin{cases}(1-\varepsilon) \frac{\left|T_{1}\right|}{|B|}, & x \in B \\ 1, & x \in I_{l} \cup I_{r} \\ \delta, & x \in T_{1} \backslash\left(B \cup I_{l} \cup I_{r}\right) .\end{cases}
$$

Again, we choose $\delta$ so that $F$ has mean value one on $T_{1}$. Then

$$
\delta=\left(\varepsilon-\frac{\left|I_{l} \cup I_{r}\right|}{\left|T_{1}\right|}\right) \frac{\left|T_{1}\right|}{\left|T_{1} \backslash\left(B \cup I_{l} \cup I_{r}\right)\right|} \geq \varepsilon / 2,
$$

say, if $Q$ is chosen large enough that $\left|I_{l} \cup I_{r}\right| /\left|T_{1}\right| \leq \varepsilon / 2$. Therefore $F$ is bounded above and below on $T_{1}$ by positive constants independent of $I$.

Notice that with this new definition of $F$ on $T_{1}$, we can still make the mean value of $X_{i} F$ on $T_{1}$ greater than $c_{1}$. For, just as for standard intervals, harmonic measure estimates show that $\left|B_{k} \cap\left(I_{l} \cup I_{r}\right)\right| /\left|T_{1}\right|$ decays exponentially in $k$, and so the contribution from $I_{l} \cup I_{r}$ to the mean value goes to zero as $Q$ goes to infinity.

This completes the proof of Lemma 14.3.

\section{5. $\mu$ IS SUPPORTED ON $L_{c}(G)$}

Define the functions $F_{n}$ as in Section 11 on all standard intervals $I \in \mathcal{H}_{n-1}$, $n \geq 1$, and as in Section 14 on all non-standard intervals $I \in \mathcal{H}_{n-1}, n \geq 1$, such that $J_{c}=\pi(\widehat{I})$ is the non-standard central Whitney interval in any component of $[0,1] \backslash K$. Define $F_{n} \equiv 1$ on the remaining grid intervals $I \in \mathcal{H}_{n-1}, n \geq 1$; these are exactly those $I \in \mathcal{H}$ such that $\pi(\widehat{I})=J_{\infty}$. We have shown that the first two types of these functions are $(\delta, \eta)$-suitable for all $I \in \mathcal{H}_{n-1}, n \geq 1$, where $\delta$ and $\eta$ are constants independent of $I$ and $n$. This is also true when $F_{n} \equiv 1$ on $I \in \mathcal{H}_{n-1}$. Therefore, by Lemma 6.3 , the measures $\mu_{n}$ defined by $d \mu_{n}=F_{n}(x) \cdots F_{1}(x) d x$ converge to a doubling measure $\mu$ on the circle.

Consider the random walk on the tree discussed in Section 4, where the vertices in the tree are the Whitney intervals $\widehat{I}$ in the boundary arcs $\bigcup_{n} \mathcal{A}_{n}$ of the half fundamental domains in our tiling of the disc; $V$ is the subset of vertices such that $\pi(\widehat{I})=J_{\infty}$ (in other words, those intervals which contain orbit points $\left.g(0), g \in G\right)$; and the probabilities of jumps between adjacent vertices are determined by the functions $F_{n}$ defined above.

In this section, we first use the estimates $E X_{i} \geq c_{1}$ and $E X_{i}^{2} \leq c_{2}$ established in Sections 13 and 14 to show that a particle starting from any vertex $v$ reaches $V(v)$ with probability one. Here $V(v)$ is the set of vertices $w \in V$ below $v$ such that there are no other vertices from $V$ between $w$ and $v$. We conclude that the 
doubling measure $\mu$ is supported on the set $S$ of points which lie in infinitely many grid intervals $I$ such that $\pi(\widehat{I})=J_{\infty}$. Finally, we show that the conical limit set of $G$ contains this set $S$, which establishes Theorem 1.2.

Let $v$ be a vertex in the tree, and $I_{0} \in \mathcal{H}_{s}$ the corresponding grid interval. Let $V\left(I_{0}\right)$ be the collection of maximal grid intervals $I \subset I_{0}$ such that $\pi(\widehat{I})=J_{\infty}$. We show that the restriction of $\mu$ to $I_{0}$ is supported on $V\left(I_{0}\right)$.

Recall that

$$
X_{i}(x)= \begin{cases}\log _{3}\left[\frac{\left|J_{i}(x)\right|}{\left.\mid J_{i-1}(x)\right]}\right], & \text { if } J_{1}(x), \ldots, J_{i-1}(x) \neq J_{\infty} \\ 1, & \text { otherwise, }\end{cases}
$$

that $S_{k}(x)=\sum_{i=1}^{k} X_{i}(x)$, and that $V\left(I_{0}\right)=\left\{x \in I_{0} \mid S_{k}(x) \rightarrow+\infty\right\}$. It is enough to show that $S_{k}(x) \rightarrow+\infty$ almost surely on $I_{0}$ with respect to $\mu$.

Let $E_{I} X_{i}$ denote the mean value of $X_{i}$ on $I$ with respect to $\mu$. We know that $\left|E_{I} X_{i}\right|<\infty$ for all grid intervals $I \in \mathcal{H}$. This is because $\left|E_{I} X_{i}\right| \leq E_{I}\left|X_{i}\right| \leq$ $E_{I} X_{i}^{2}<c_{2}$, since $X_{i}$ is integer-valued. Define new functions $\widetilde{X}_{i}(x)$ on $I_{0}$ which have mean zero on each $I \in \mathcal{H}_{s+i-1}$ which lies in $I_{0}$ :

$$
\begin{aligned}
\tilde{X}_{1}(x) & =X_{1}(x)-E_{I_{0}} X_{1} \\
\widetilde{X}_{i}(x) & =\sum_{I \in \mathcal{H}_{s+i-1}, I \subset I_{0}}\left(X_{i}(x)-E_{I} X_{i}\right) \chi_{I}(x),
\end{aligned}
$$

for $i \geq 1$, and let

$$
\begin{aligned}
\widetilde{S}_{k}(x) & =\sum_{i=1}^{k} \widetilde{X}_{i}(x) \\
& =\sum_{i=1}^{k}\left(X_{i}(x)-E_{I_{i-1}} X_{i}\right)
\end{aligned}
$$

where $x \in I_{i-1}, i \geq 1$.

Then $\left\{\widetilde{S}_{k}\right\}$ is a martingale with respect to $\mu$.

Also, $E \widetilde{X}_{i}^{2}$ is bounded by $E X_{i}^{2}$, which is bounded by $c_{2}$. Therefore, by the strong law of large numbers for martingales [Fel], $\widetilde{S}_{k} / k \rightarrow 0$ almost surely on $I_{0}$ with respect to $\mu$.

Now let $x$ be a point in $I_{0}$ such that $\widetilde{S}_{k}(x) / k \rightarrow 0$, and let $I_{i} \in \mathcal{H}_{s+i}$ be the interval containing $x$, for $i \geq 0$. Then

$$
\begin{aligned}
\frac{S_{k}(x)}{k} & =\frac{1}{k} \sum_{i=1}^{k} X_{i}(x) \\
& =\frac{1}{k} \sum_{i=1}^{k}\left(\widetilde{X}_{i}(x)+E_{I_{i-1}}^{d \mu} X_{i}\right) \\
& \geq \frac{1}{k} \widetilde{S}_{k}(x)+c_{1} \\
& \geq c_{1} / 2,
\end{aligned}
$$

for all $k$ sufficiently large that $\left|\widetilde{S}_{k}(x) / k\right| \leq c_{1} / 2$. Therefore $S_{k}(x) \geq c_{1} k / 2$ for all such $k$, and so $S_{k}(x) \rightarrow+\infty$. Thus $S_{k} \rightarrow+\infty$ almost surely with respect to $\mu$ on 


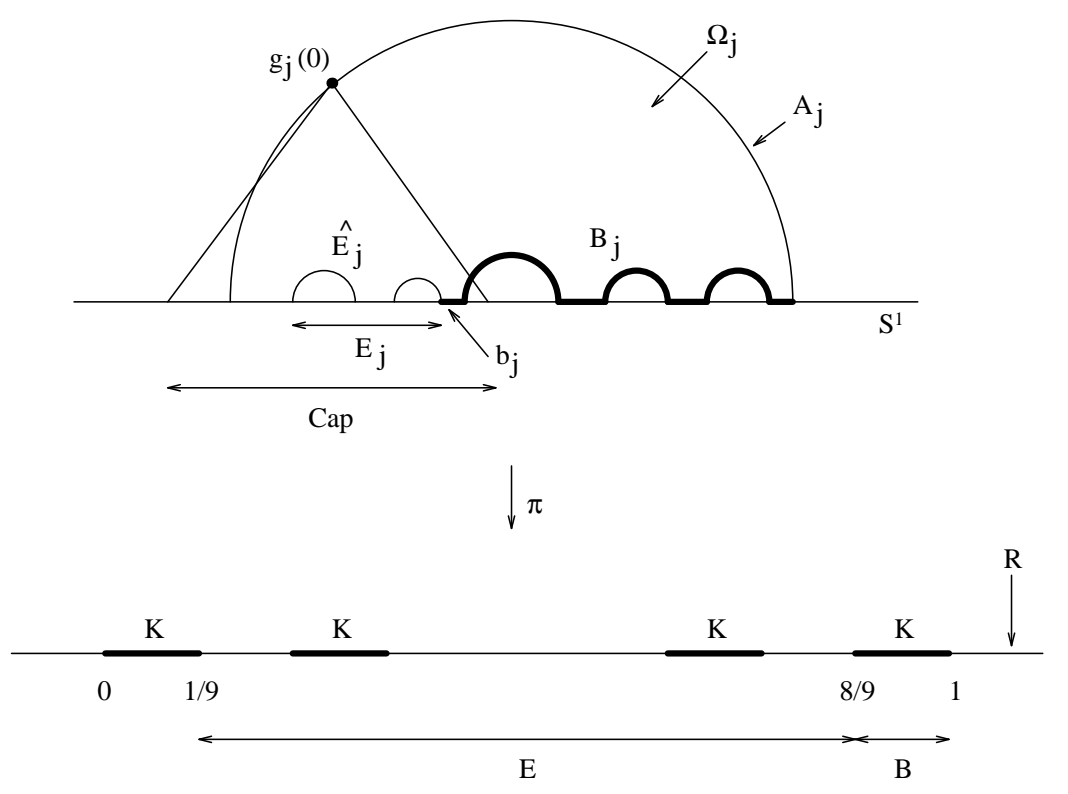

Figure 14. $P\left(\widehat{I_{j}}\right)=E_{j}$ lies in Cap $=\operatorname{Cap}\left(g_{j}(0), l\right) ; E=E_{J_{\infty}}$.

$I_{0}$. Since $V\left(I_{0}\right)$ is exactly the set of points $x$ in $I_{0}$ such that $S_{k} \rightarrow+\infty$, this implies that $\mu\left(V\left(I_{0}\right)\right)=\mu\left(I_{0}\right)$.

Since a particle starting from any vertex $v$ reaches $V(v)$ with probability one, it is now clear that a particle starting anywhere in the tree will pass through infinitely many vertices in $V$, with probability one. (Note that we have now defined the only jump probabilities which were missing; these are the probabilities of the jumps from vertices in the subset $V$, and they are determined by the functions $F_{n} \equiv 1$ on the corresponding grid intervals.) Thus we have shown that the doubling measure $\mu$ is supported on the set $S$ of points in the circle which lie in infinitely many grid intervals $I=P(\widehat{I})$ such that $\pi(\widehat{I})=J_{\infty}$.

It remains to show that $S$ is contained in the conical limit set of $G$. We show that there is a uniform constant $l$ such that for each orbit point $g_{j}(0) \neq 0$, the Whitney interval $\widehat{I}_{j}$ which contains $g_{j}(0)$ projects to a grid interval $P\left(\widehat{I}_{j}\right)$ which lies in the spherical cap Cap $\left(g_{j}(0), l\right)$ on $g_{j}(0)$. Then each point $x \in S$ lies in infinitely many of these caps, and therefore in the conical limit set.

By definition, the tip of the leaf based at $J_{\infty}$ is $E_{J_{\infty}}=[1 / 9,8 / 9]$. For each $g_{j} \in G \backslash\{\operatorname{id}\}$ let $A_{j}$ be the orthocircular arc in $\bigcup_{n} \mathcal{A}_{n}$ which contains $g_{j}(0)$, and let $\Omega_{j}$ be the half fundamental domain below $A_{j}$. Let $\mathcal{F}_{j}$ be the fundamental domain consisting of $\Omega_{j}$ and the half fundamental domain above $A_{j}$. Let $\widehat{E_{j}}$ be the segment of $\partial \Omega_{j}$, below $g_{j}(0)$, such that $\pi\left(\widehat{E_{j}}\right)=E_{J_{\infty}}$. The endpoints of $\widehat{E_{j}}$ lie in $\partial \mathbb{D}$. Then $P\left(\widehat{I}_{j}\right)=E_{j}$ is the arc of $\partial \mathbb{D}$ below $\widehat{E_{j}}$ (with the same endpoints as $\widehat{E_{j}}$ ). See Figure 14.

Lemma 15.1. There is a constant $l>0$ such that for all $j$,

$$
P\left(\widehat{I_{j}}\right) \subset \operatorname{Cap}\left(g_{j}(0), l\right) .
$$


Proof. By definition,

$$
\operatorname{Cap}\left(g_{j}(0), l\right)=\left\{z \in \partial \mathbb{D} \mid \operatorname{dist}\left(z, g_{j}(0)\right) \leq l\left(1-\left|g_{j}(0)\right|\right)\right\} .
$$

Let $z_{j}=g_{j}(0)$. Let $b_{j} \in \partial \mathbb{D}$ be the endpoint of $P\left(\widehat{I_{j}}\right)$ which is furthest from $z_{j}$. We show that $\left|z_{j}-b_{j}\right| /\left(1-\left|z_{j}\right|\right) \leq c$.

If $b_{j}$ satisfies $\left|b_{j}-z_{j}\right| \leq 10\left(1-\left|z_{j}\right|\right)$, we are done. If not, let $B_{j}$ be the component of $\pi^{-1}\left([0,1] \backslash E_{J_{\infty}}\right)$ which has $b_{j}$ as an endpoint; and let $B=\pi\left(B_{j}\right)$. See Figure 14 for one possible configuration.

First, $\left|z_{j}-b_{j}\right| \leq c$ dist $\left(z_{j}, B_{j}\right)$. For if $a$ is any point in $B_{j} \cap \partial \mathbb{D}$, then $\left|z_{j}-b_{j}\right| \leq$ $\left|z_{j}-a\right|$, while if $a$ is in $B_{j} \backslash \partial \mathbb{D}$, then $a$ lies in one of the orthocircular arcs in $\partial \mathcal{F}_{j}$, and $\left|z_{j}-a\right| \geq c\left|z_{j}-b_{j}\right|$.

By Beurling's lemma (see also (10.12)),

$$
\begin{aligned}
\operatorname{dist}\left(z_{j}, B_{j}\right) & \leq c \frac{\operatorname{dist}\left(z_{j}, \partial \mathcal{F}_{j}\right)}{\omega\left(z_{j}, B_{j}, \mathcal{F}_{j}\right)^{2}} \\
& \leq c \frac{\operatorname{dist}\left(z_{j}, \partial \mathcal{F}_{j}\right)}{\omega\left(\pi\left(z_{j}\right), \pi\left(B_{j}\right), \pi\left(\mathcal{F}_{j}\right)\right)^{2}} \\
& =c \frac{\operatorname{dist}\left(z_{j}, \partial \mathcal{F}_{j}\right)}{\omega(\infty, B, \overline{\mathbf{C}} \backslash K)^{2}} \\
& \leq c \operatorname{dist}\left(z_{j}, \partial \mathcal{F}_{j}\right) \\
& \leq c\left(1-\left|z_{j}\right|\right) .
\end{aligned}
$$

We have shown that each point in $\partial \mathbb{D}$ which is at the end of a path which passes through infinitely many orbit points is actually a conical limit point of $G$, in particular, that the orbit points on the path not only accumulate at the endpoint but lie in some non-tangential cone based at the endpoint. Therefore the set $S$ lies in the conical limit set of $G$.

This completes the proof of Theorem 1.2.

\section{REFERENCES}

[A] L.V. Ahlfors, Conformal Invariants: Topics in Geometric Function Theory, McGrawHill, New York, 1973. MR 50:10211

[BP] A. Beardon and Ch. Pommerenke, The Poincaré metric of a plane domain, J. London Math. Soc. 18 (2) (1978), 475-483. MR 80a:30020

[BA] A. Beurling and L. Ahlfors, The boundary correspondence under quasiconformal mappings, Acta Math. 96 (1956), 125-142. MR 19:258c

[BJ] C. Bishop and P.W. Jones, Hausdorff dimension and Kleinian groups, Acta Math. 179 (1997), 1-29. CMP 98:05

[DE] A. Douady and C.J. Earle, Conformally natural extension of homeomorphisms of the circle, Acta Math. 157 (1986), 23-48. MR 87j:30041

[F] J.-L. Fernández, Domains with strong barrier, Revista Matemática Iberoamericana 5 (1989), 47-65. MR 91j:30016

[Fel] W. Feller, "An Introduction to Probability Theory and Its Applications," Volume II, Wiley, New York, 1966. MR 35:1048

[GGJ] J.B. Garnett, F.W. Gehring and P.W. Jones, Quasiconformal groups and the conical limit set, in "Holomorphic Functions and Moduli II," ed. D. Drasin et al., MSRI Publ. 11, Springer-Verlag, 1986, 59-67. MR 89i:30021

[G] M.-J. González, Uniformly perfect sets, Green's function, and fundamental domains, Revista Matemática Iberoamericana 8 (1992), 239-269. MR 93k:30029

[He] G.A. Hedlund, Fuchsian groups and transitive horocycles, Duke Math. J. 2 (1936), 530542 . 
[H] E. Hopf, Fuchsian groups and ergodic theory, Trans. Amer. Math. Soc. 39 (1936), 299314.

[JK] D.S. Jerison and C.E. Kenig, Hardy spaces, $A_{\infty}$, and singular integrals on chord-arc domains, Math. Scand. 50 (1982), 221-247. MR 84k:30037

[K] J.-P. Kahane, Trois notes sur les ensembles parfaits linéaires, Enseignement Math. 15 (1969), 185-192. MR 29:7040

[Kam1] S. Kamiya, Discrete subgroups of convergence type of $U(1, n ; \mathbf{C})$, Hiroshima Math. J. 21 (1991), 1-21. MR 93e:22020a

[Kam2] S. Kamiya, Notes on discrete subgroups of convergence type of $U(1, n ; \mathbf{C})$, Math. Japonica 38 (1993), 583-587. MR 94h:30055

[L] O. Lehto, "Univalent Functions and Teichmüller Spaces," Springer-Verlag, Graduate Texts in Mathematics 109, 1987. MR 88f:30073

[M] K. Matsuzaki, Ergodic properties of discrete groups; inheritance to normal subgroups and invariance under quasiconformal deformations, J. Math. Kyoto Univ. 33 (1993), No. 1, 205-226. MR 94m:30083

[MW] G.J. Martin and L. Ward, On the conical limit set of a complex hyperbolic group, Mathematics Research Report No. MRR 046-95, Centre for Mathematics and its Applications, Australian National University, 1995.

[N1] P.J. Nicholls, The limit set of a discrete group of hyperbolic motions, in 11 Holomorphic Functions and Moduli II," ed. D. Drasin et al., MSRI Publ. 11, Springer-Verlag, 1986, 141-164. MR 89h:30060

[N2] P.J. Nicholls, A measure on the limit set of a discrete group, in "Ergodic Theory, Symbolic Dynamics and Hyperbolic Spaces," ed. T. Bedford et al., Oxford University Press, 1991, 259-297. CMP 92:02

[Pa1] S.J. Patterson, The limit set of a Fuchsian group, Acta Math. 136 (1976), 241-273. MR 56:884

[Pa2] S.J. Patterson, Spectral theory and Fuchsian groups, Math. Proc. Camb. Phil. Soc. 81 (1977), 59-75. MR 56:5435

[Po1] Ch. Pommerenke, On the Green's function of Fuchsian groups, Ann. Acad. Sci. Fenn. Ser. A. I. Math. 2 (1976), 409-427. MR 57:6412

[Po2] Ch. Pommerenke, Uniformly perfect sets and the Poincaré metric, Arch. Math. 32 (1979), 192-199. MR 80j:30073

[Po3] Ch. Pommerenke, "Boundary Behavior of Conformal Maps," Springer-Verlag, Berlin, 1992.

[RR] L. Rubel and J. Ryff, The bounded weak-star topology and bounded analytic functions, J. Funct. Anal. 5 (1970), 167-183. MR 40:7788

[S1] D. Sullivan, The density at infinity of a discrete group of hyperbolic motions, Publ. Math. I.H.E.S. 50, 171-202. MR 81b:58031

[S2] D. Sullivan, On the ergodic theory at infinity of an arbitrary discrete group of hyperbolic motions, in "Riemann surfaces and related topics: Proceedings of the 1978 Stonybrook Conference," Annals of Math. Studies 97, Princeton Univ. Press, 1981, 465-496. MR 83f: 58052

[T1] P. Tukia, On two-dimensional quasiconformal groups, Ann. Acad. Sci. Fenn. Ser. A. I. Math. 5 (1980), 73-78. MR 82c:30031

[T2] P. Tukia, A quasiconformal group not isomorphic to a Möbius group, Ann. Acad. Sci. Fenn. Ser. A. I. Math. 6 (1982), 149-160. MR 83b:30019

[T3] P. Tukia, On quasiconformal groups, J. d'Analyse Math. 46 (1986), 318-346. MR 87 m:30043

[V] J. Väisälä, Quasimöbius maps, J. d'Analyse Math. 44 (1984/85), 218-234. MR 87f:30059

[W] L. Ward, Fuchsian groups, quasiconformal groups, and conical limit sets, Ph.D. thesis, Yale University, 1994.

Department of Mathematics, Yale University, New Haven, Connecticut 06520-8283

E-mail address: jones@math.yale.edu

Department of Mathematics, Rice University, Houston, Texas 77005-1892, and MSRI, 1000 Centennial Drive, Berkeley, California 94720-5070

Current address: Department of Mathematics, Harvey Mudd College, Claremont, California 91711

E-mail address: ward@math.hmc.edu 